University of San Diego

Digital USD

Theses

Theses and Dissertations

Spring 5-31-2018

\title{
The Impact of Copper on Non-Indigenous and Native Species of Suspension-Feeding Bivalves in Mission Bay, San Diego, California
}

Brian Juhl

University of San Diego

Follow this and additional works at: https://digital.sandiego.edu/theses

Part of the Marine Biology Commons

\section{Digital USD Citation}

Juhl, Brian, "The Impact of Copper on Non-Indigenous and Native Species of Suspension-Feeding Bivalves in Mission Bay, San Diego, California" (2018). Theses. 28.

https://digital.sandiego.edu/theses/28

This Thesis: Open Access is brought to you for free and open access by the Theses and Dissertations at Digital USD. It has been accepted for inclusion in Theses by an authorized administrator of Digital USD. For more information, please contact digital@sandiego.edu. 
University of San Diego

San Diego

The Impact of Copper on Non-Indigenous and Native Species

of Suspension-Feeding Bivalves in

Mission Bay, San Diego, California

A thesis submitted in partial satisfaction of the requirements for the degree of

Master of Science in Marine Science

by

Brian Wellerson Juhl

Thesis Committee

Ronald Kaufmann, PhD, Committee Chair

Jeff Crooks, PhD, Committee Member

Ignacio Rivera-Duarte, PhD, Committee Member 
The thesis of Brian Wellerson Juhl is approved by:

Ronald Kaufmann, PhD, Committee Chair

Jeff Crooks, PhD, Committee Member

Ignacio Rivera, PhD, Committee Member

University of San Diego

San Diego

2018 
Copyright 2018

Brian Juhl 


\section{Acknowledgments}

This thesis was the culmination of years of hard work, more years than expected when I started, and I count it as one of the most difficult and yet rewarding things I have worked on in my life. This accomplishment would not have been achieved were it not for the help and guidance of many people that assisted and encouraged me along the way. I would like to first thank the members of my committee, Dr. Ron Kaufmann, Dr. Jeff Crooks, and Dr. Ignacio Rivera-Duarte for their advice and help giving me different perspectives on this project. Through their guidance, I have been able to navigate my way to the completion of this thesis, keeping high scientific standards for my work. I especially would like to thank my chair, Ron Kaufmann, for the support he's given to me throughout the years of work on this project, as I know that he has put in a great deal of work into this thesis and has given me invaluable advice as I worked my way through this project.

I'd also like to thank the friends that I made in the Marine Science Program for supporting me through the frustrating times of my work, even as they faced their own problems with their respective theses. I'd especially like to thank Kevin Stolzenbach, Whitney Sears, and Morgan Martin for their friendship during this time, as having friends and peers of the quality that I had during my time here not only motivated me to raise the quality of my work, but also gave me some of the most memorable, enjoyable times of my life through our shared experiences.

My family has also been instrumental in my success, giving me support and encouragement to push on through the more challenging times. I'd like to thank my 
wife, Kristin, for always being there to listen to my frustrations and for helping to motivate me to finally finish what has been almost six years of work.

Finally, I'd like to thank the University of San Diego, and the Marine Science program for providing me the material support needed to conduct this study, through both employment and facilities and resources for this project. 


\section{TABLE OF CONTENTS}

\section{Chapter 1: Introduction}

$\begin{array}{lll}\text { 1.1.1 Overview and research questions } & 1\end{array}$

1.1.2 Non-indigenous species and the nature of invasions 4

1.1.3 Vulnerability to invasion and biotic resistance 5

$\begin{array}{lll}\text { 1.1.4 Environmental tolerance } & 7\end{array}$

1.1.5 Native species in Mission Bay 9

$\begin{array}{ll}\text { 1.1.6 Non-indigenous species in Mission Bay } & 10\end{array}$

1.1.7 Copper as an environmental toxin 12

1.1.8 Copper chemical speciation in water column and free copper $\left(\mathrm{Cu}^{2+}\right)$

$\begin{array}{lll}\text { 1.1.9 Biological effects of copper } & 15\end{array}$ 
1.1.10 How this project addresses the effects of copper on non-indigenous and native species

Chapter 2: The Impact of Copper on Non-Indigenous and Native Species of Suspension-Feeding Bivalves in Mission Bay, San Diego, California

2.2.2 Experiment Design, Depuration, and Tank Setup

2.2.3 Algae Depletion Setup and Procedure

2.2.4 Survivorship and Post-Experiment Growth Measurement

2.2.6 Tissue Copper: Dissection, Digestion, and Analysis 30

2.2.7 Statistical Methods

2.3 Results 
2.4 Discussion

2.4.1 Water Quality

121

2.4.2 Copper Accumulation

2.4.3 Survivorship

2.4.4 Growth

2.4.5 Mucus Production 139

2.4.6 Algae Depletion

\section{Chapter 3: Conclusions}

3.1 Conclusions

3.1.1 Future Research

3.2 Literature Cited

\section{Appendices}

Appendix A: Mission Bay

Appendix B: Tables

171

Appendix C: Results Comparison with Literature

179

Appendix D: Pilot Study Data

183 


\section{List of Figures}

Figure 1.1: Diagram of the experimental design and setup of the algae depletion experiment

Figure 1.2: Estimated vs. measured copper concentrations across both experiments

Figure 1.3: Visual layout of randomized experimental design

Figure 1.4: Mean dissolved copper concentrations for

initial water samples and difference between initial copper concentration

and copper concentration at water change

Figure 2.1: Mean copper concentration per gram dry mass of specimens

from the 18 day experiment 
Figure 2.2: Mean copper concentration per gram dry mass of specimens

from the 12 day experiment

Figure 3.1: Survivorship percentages over experiment duration

Figure 3.2: Survivorship percentage over time for 18 day exposure

of all species at various estimated copper exposure levels.

Figure 3.3: Survivorship percentage over time for 18 day exposure of all species at various estimated copper exposure levels.

Figure 3.4: Survivorship percentage over time for 12 day exposure of all species at various estimated copper exposure levels.

Figure 3.5: Survivorship percentage over time for 18 day exposure of all species at various estimated copper exposure levels. 
Figure 3.6: Mean survivorship percentage of Chione californiensis,

Venerupis philippinarum, and Musculista senhousia compared to mean

copper per dry mass of whole body, gills and digestive tract

for individuals in the 18 day exposure experiment

67

Figure 3.7: Mean survivorship percentage of Chione californiensis, Venerupis

philippinarum, and Musculista senhousia compared to mean

copper per dry mass of whole body, gills and digestive tract

for individuals in the 12 day exposure experiment

Figure 3.8: Survivorship percentages over time for each tank in the 18 day

experiment, including all species at various estimated copper exposure levels.

Figure 3.9: Survivorship percentages over time for each tank in the 12 day

experiment, including all species at various estimated copper exposure levels. 73 
Figure 4.1: Measurements for growth percentage and overall growth

for 12 day experiment

80

Figure 4.2: Mean growth percentage compared to mean copper

per dry mass of whole body and dissected organs for specimens in the

12 day exposure experiment.

82

Figure 4.3: Relationship between growth percentage and mean copper

per dry mass of whole body for specimens in the 12 day exposure experiment. 84

Figure 5.1: Mucus/Dry Mass ratio for Chione californiensis,

Venerupis philippinarum, and Musculista senhousia.

Figure 5.2: Mean mucus/dry mass ratio compared to mean copper per

dry mass of whole body and dissected organs for specimens

in the 18 day exposure experiment

94 
Figure 5.3: Mean mucus/dry mass ratio compared to mean copper

per dry mass of whole body and dissected organs for specimens in

the 12 day exposure experiment

Figure 5.4: Mucus/dry mass ratio compared to copper per dry mass of whole body for the 18 day and the 12 day exposure experiments

Figure 6.1: Control subtraction representation of mean algae depletion rates

for 18 day experiment by species

Figure 6.2: Control subtraction representation of mean algae depletion rates

for 18 day experiment by concentration

Figure 6.3: Control subtraction representation of mean algae depletion rates

for 12 day experiment by species 
Figure 6.4: Control subtraction representation of mean algae depletion rates

for 12 day experiment by concentration

Figure 6.5: $0 \mathrm{ppb}$ subtraction representation of mean algae depletion rates

for 18 day experiment by concentration

Figure 6.6: $0 \mathrm{ppb}$ subtraction representation of mean algae depletion rates

for 18 day experiment by species

Figure 6.7: $0 \mathrm{ppb}$ subtraction representation of mean algae depletion rates

for 12 day experiment by concentration

Figure 6.8: $0 \mathrm{ppb}$ subtraction representation of mean algae depletion rates

for 12 day experiment by species 


\section{List of Tables}

\section{APPENDIX B}

Table 1.1 Layout of staggered experiment initiation schedule

171

Table 2.1: Tissue copper data for 18 day and 12 day experiments

172

Table 3.1: Survivorship by tank data for 18 day and 12 day experiments

174

Table 4.1: Overall growth and growth percentage data for 18 day

and 12 day experiments

Table 5.1: Mucus/Dry mass ratio data for 18 day and 12 day experiments

Table 6.1: Unaltered algae depletion data for 18 day and 12 day experiments 


\section{APPENDIX C}

Table 7.1: Tissue copper accumulation comparison between this study and previously published research.

Table 7.2: Survivorship comparison between this study and previously published research.

Table 7.3: Growth comparison between this study and previously published research.

Table 7.4: Mucus production comparison between this study and previously published research.

\section{APPENDIX D}

Table 8.1: Pilot study comparing survivorship at different copper exposures across species. 


\begin{abstract}
Native (Chione californiensis) and non-indigenous bivalve species [Musculista senhousia (now known as Arcuatula senhousia), and Venerupis philippinarum] were collected from Mission Bay, San Diego, California and exposed to copper contaminated water at estimated concentrations of $0 \mathrm{ppb}, 25 \mathrm{ppb}$, and $50 \mathrm{ppb}$ over the course of two experimental periods of 18 days and 12 days. Bivalves were placed in tanks in a temperature controlled environment, and measurements of a number of parameters were taken during and after the exposure to copper: survivorship, feeding rates, growth, mucus production, and tissue copper concentrations for the entire body, gills, and digestive tract. These factors were analyzed on their own and in comparison to tissue copper concentration to examine potential relationships. Observations indicated that there were several statistically significant differences, either between copper concentration groups, between species, or both. The majority of sublethal measures showed no statistically significant differences across both experiments, with the exception of whole body copper accumulation. Whole body copper accumulation showed significant differences between Musculista and both Chione and Venerupis, and may suggest a competitive disadvantage in copper contaminated environments, as Chione and Venerupis may be more efficient at removing copper from tissue. Other sublethal measures that did show significance did not show consistency across both experiments or showed contradicting trends, and none showed consistently significant trends when compared with tissue copper concentrations. Algae depletion rate measurements produced inconclusive results. The data indicates that most sublethal biomarkers used in this experiment were not indicators for copper toxicity for the species in this experiment at the tested concentrations, and that of those species,


Chione was the most resistant to the effects of copper with respect to mortality and whole body accumulation. 


\section{Chapter 1: Introduction}

\subsection{Introduction}

\subsubsection{Overview and research questions}

Introduction of non-indigenous species (NIS) is a growing problem for ecosystems around the world. In marine communities, competition for resources such as food or space and increased predation on native prey species are two of the direct effects of NIS invasions (Piola et al. 2009, Crooks et al. 2011), but there are indirect effects as well, such as an increase in predator biomass when a non-indigenous prey species is introduced (Byers 2005). Human activities, such as pollution and habitat alteration, have been shown to increase the vulnerability of an ecosystem to invasion (Crooks and Suarez 2006). The potential for some NIS to have higher survival rates than native species in contaminated environments could have far-reaching consequences, as some research has shown a drastic reduction in native populations as non-indigenous species proliferate.

Some NIS, including bivalves and arthropods, may have a higher tolerance for pollutants such as copper, in comparison to native species (Crooks et al. 2011, Piola and Johnston 2009). Elevated concentrations of copper can have a negative impact on survivorship of many species, including suspension-feeding bivalves (Al-Subiai et al. 2011, Crooks et al. 2011). Copper also can have sublethal effects that may place stress on bivalve species, reducing the viability of populations through reduced growth rates and lowered reproduction in a contaminated environment (Al-Subiai et al. 2011, Vosloo et al. 2012). 
Mission Bay, San Diego CA, has native species and NIS, as well as a copper concentration gradient. Mission Bay is a mesotidal Mediterranean-type estuary in Southern California (Largier 1997, Crooks 2001). Human activities in the watersheds surrounding Mission Bay release anthropogenic pollution that may make Mission Bay more vulnerable to biological invasions. Elevated dissolved copper concentrations have been measured in different locations in Mission Bay, with the highest concentrations in the back of the bay (Kaufmann et al. 2004). Several non-indigenous species of suspension-feeding bivalves have been observed in the Mission Bay ecosystem. In particular, the Asian mussel, Musculista senhousia (now known as Arcuatula senhousia), has been shown to have a negative impact on populations of native suspension-feeding bivalve species, such as Chione undatella and Chione fluctifraga (Crooks 2001). This project studies how dissolved copper concentrations affect feeding and growth rates of non-indigenous and native species of suspension-feeding bivalves from Mission Bay and examine the degree to which NIS and native species of bivalves are affected by dissolved copper.

In this thesis, the following questions are addressed:

1.) How do environmental copper concentrations correlate with rates of survivorship, growth, filtration and mucus production in native vs. nonindigenous species of suspension-feeding bivalves from Mission Bay?

2.) How does environmental copper concentration correlate with copper accumulation across the whole body tissue, gill tissue, and digestive tract tissue 
between native vs. non-indigenous species of suspension feeding bivalves from Mission Bay?

3.) Is there a statistically significant correlation between tissue copper levels and survivorship, growth, filtration, and mucus production, and is there a difference in this relationship between non-indigenous and native species?

In this introduction, background information is discussed in relation to the research project. First, the issues of non-indigenous species and various aspects of their dispersal and ecosystem impact are discussed, examining past studies that have shown how NIS have spread, why they are able to sustain populations in new environments, and the effects that their presence can have on native species and ecosystems as a whole. The principles of environmental vulnerability and biotic resistance are examined to further understand the mechanics of an invasion by NIS and what factors play a role in the success or failure of an invasion. Then, the topic of environmental tolerance is explored as one of the factors that play a role in the success of species when exposed to different environmental conditions. Mission Bay and the properties that make it vulnerable to invasive species are then discussed to illustrate an example of a coastal environment undergoing invasion and the factors involved with the facilitation of invasions. Next, profiles of each of the species used in this experiment were created to closely examine the current research and traits of the test species. Following these profiles, different aspects of copper as an environmental toxin are examined in turn, from biological impact to the different forms that copper can take in the water column. 
Finally, the section concludes with a summary of the purpose of this thesis project and how the project addresses the impact of copper on NIS and native species.

\subsubsection{Non-indigenous species and the nature of invasions}

Invasion by non-indigenous species has been shown to have the capacity to harm ecosystems (Elton 1958, Dexter and Crooks 2000, Crooks 2006, Johnston et al. 2009). In this thesis, the term "non-indigenous species" (NIS) will be used when referring to species that are not native to a particular area. Non-indigenous species are defined by the National Oceanic and Atmospheric Association (NOAA) as species that are found living beyond their native historical range. The introduction of NIS into an ecosystem can lead to several direct impacts on native species. First, non-indigenous species compete with native species that rely on the same resources to survive. The competition for resources between native and NIS can have a negative effect on native species, especially when NIS are better adapted to the environment (Crooks 2001, Dafforn et al. 2009, Crooks et al. 2011). This situation often leads to a population increase of the NIS and/or a population decrease or eradication of the native species. This has been reported for the Musculista senhousia invasion of Mission Bay (Crooks 2001), where the combination of lowered survival and growth rates in native species and decreased species richness and diversity observed by Crooks (2001) illustrates the severity of the impact that some NIS can have on native species. Second, the presence of NIS, if they are carnivorous, means that local prey species will have an additional predator in the ecosystem, negatively impacting native prey populations. 
Species may be introduced to new areas in a variety of ways. While some species may enter new areas through dispersal, many invasions have had humanmediated origins. Examples of this include intentional introduction to an area for commercial purposes, release of non-indigenous species that are pets and accidental transport. Intentional introduction to an area can happen with a variety of motivations, but usually the main two are as a food source (as was the case with Crassostrea gigas; Troost 2004) or as a biological control for a pest species, such as the introduction of Trichogramma ostrinae to control populations of the European corn borer, Ostrinia nubilalis, in the northeastern United States (Wright et al. 2005). An example of a pet release resulting in invasion was the release of Burmese pythons in Florida, which has had a negative effect on native prey populations in the Everglades (Wilson et al. 2011). Accidental transport is the most common method of introducing non-indigenous species to aquatic environments, typically through attachment to boats or as larvae in ballast water. The presence of multiple NIS also may have indirect effects, such as alteration of the environment and effects on the populations of other species within the ecosystem (Montgomery et al. 2012). There is evidence that the presence of multiple NIS can lead to an "invasion meltdown," in which the impact of a single NIS is exacerbated by interactions with other NIS (Simberloff et al. 1999).

\subsubsection{Vulnerability to invasion and biotic resistance}

Biotic resistance is defined as an ecosystem's natural barriers that prevent the establishment of an invasive species (Elton 1958, Cheng and Hovel 2009). Areas that have low species diversity are more susceptible than systems with high natural diversity 
(Rejmanek 1989). Having a diverse suite of native competitors for resources can limit populations and curtail the effects of non-indigenous species (Elton 1958, Reusch 1998). Ecosystems that do not have existing predators or competitors for the non-indigenous species are especially vulnerable to invasion (Rejmanek 1989). Cheng and Hovel (2009) showed that the distribution of Musculista senhousia in Mission Bay was influenced by the presence of natural predators, such as gastropods, but this influence was dependent on location. Reusch (1998) observed that the muricid snail, Pteropurpura festiva, negatively impacted Musculista senhousia populations within eelgrass beds in San Diego Bay and was an example of a native predator that adapted to use a NIS as a preferred food source over native species. Venerupis philippinarum, another NIS in some systems, also was shown to be favored as prey over native species. However, it has been speculated that the presence of Venerupis philippinarum still had a negative impact on native species, because the high abundance of Venerupis philippinarum contributed to an overall increase in predator biomass (Byers 2005).

Ecosystems that have been disturbed and degraded by human activity have been shown to be especially susceptible to invaders, because anthropogenic activity can degrade biotic resistance factors (Piola and Johnston 2008). For instance, in an analysis of vulnerability of lakes to zebra mussel invasion, it was found that changes in $\mathrm{pH}$ and calcium concentrations of an ecosystem increased the vulnerability of that area to invasion (Drake and Bossenbroek 2009). There is also a natural susceptibility of certain environments to invasion. In the aquatic realm, pelagic marine ecosystems are least susceptible, while near-shore marine systems appear to be among the most susceptible, based on the numbers of NIS that have been observed in those environments (Heywood 1995). This is likely due to higher propagule pressure found in coastal environments in 
comparison with the open ocean, in which more individuals of the invasive species are exposed to an area. Coastal environments are more accessible to humans than the open ocean, and thus more accidental transport and other human-mediated invasion methods are likely.

\subsubsection{Environmental tolerance}

The success of NIS across different environments raises the question of how they are able to survive the biotic resistance mechanisms of ecosystems. This success can be traced to several different sources, depending on the species. Successful invasions can be linked to ecosystem type such as the difference between invasion success rate in coastal vs. pelagic marine environments (Heywood 1995). There are also adaptations by NIS that have an impact on establishment success. Most successful NIS exhibit some combination of the following traits: resistance to predation/lack of predators, high tolerance for a range of different environmental conditions, high levels of reproductive success and/or large numbers of offspring, fast growth rates, successful dispersal methods, and efficient utilization of resources (Richardson and Pysek, 2006). NIS often face high levels of stress during entrainment and transport from one area to another (Johnston et al. 2009), and the invasion process is thought to favor species that have broad environmental tolerances. For instance, Musculista senhousia has exhibited higher recruitment levels and higher tolerance to contaminants than native bivalve species, which allow them to rapidly increase their numbers and better survive in chemically-contaminated environments (Crooks 1998b, Crooks 2001, Crooks et al. 2011). 
There is evidence that NIS exhibit higher tolerance to other environmental conditions as well. Lenz et al. (2011) conducted a stress test of marine invertebrate species, including the bivalves Crassostrea gigas and Perna perna, that spanned multiple native and NIS from temperate, tropical, and subtropical areas. NIS exhibited higher survivorship and lower deviation in respiratory patterns under stress for temperature, salinity, and oxygen concentration than comparable native species (Lenz et al. 2011). Similarly, non-indigenous species of freshwater mollusks such as Corbicula fluminalis and Physella acuta, showed significantly higher temperature tolerance compared to native species, though there was no significant difference in salinity tolerance (Verbrugge et al. 2012). The physiological mechanism that produces this capacity may involve the ability to induce changes in the expression of different genes and proteins in response to temperature stress, as has been shown in Mytilus galloprovincialis (Lockwood et al. 2011). If this ability exists in other invaders, it could explain why nonindigenous species often exhibit higher temperature tolerances.

Chemical pollutants are known to affect the balance between native and nonindigenous species diversity. Increased pollutant loads were shown to alter hardsubstrate harbor environments previously dominated by native species (Piola and Johnston 2008). Native hard-substrate organisms such as bryozoans, sponges, and barnacles went from dominance to either being dominated by NIS of those same taxa or to having the space equally occupied by NIS and native species (Piola and Johnston 2008). Copper is a common environmental toxin in marine systems, due to its frequent introduction to areas such as bays and estuaries through human activity (Neira et al. 2009). Non-indigenous fouling species across a range of taxa, including bivalves, sponges, and bryozoans, had higher survival rates when treated with copper compared 
to native species of the same taxa (Crooks et al. 2011). These results supported an earlier study of copper tolerance by invasive and native bryozoan species, which also showed higher survival rates for invaders (Piola et al. 2009).

\subsubsection{Native species in Mission Bay}

Chione californiensis, Chione undatella and Chione fluctifraga

The frilled venus, Chione undatella, the California venus, Chione californiensis, and the smooth venus, Chione fluctifraga, are suspension-feeding bivalves that are native to intertidal areas in Mission Bay. These species have been shown to be sensitive to the introduction of NIS. Following the invasion of Musculista senhousia, densities of

C. fluctifraga declined in creek areas in the Northern Wildlife Preserve (Crooks 2001). C. undatella began appearing in this creek area but declined in the other areas of Mission Bay where they had been collected previously (Crooks 2001). C. undatella is a hardy bivalve with a long lifespan and is relatively sedentary (Peterson 1982). This lack of mobility may cause $C$. undatella to stay in a polluted area for long periods of time due to lack of mobility and experience heavy contamination (Peterson 1982). C. fluctifraga can withstand high concentrations of suspended organic matter as well as a wide range of temperatures and salinities (Martínez-Córdova, 1988). Largely found in estuaries, this species has a short life cycle and can reproduce in a period of a few months (MartínezCórdova, 1988). C. californiensis is the third species of Chione that is commonly found in Mission Bay, living in the intertidal zone (Tuskes, 2012). It also has been shown to be abundant in the Gulf of California, where there is a fishery for this species (Garcia- 
Dominguez et al., 1993). Little research has been done on this species, as reflected in a dearth of scientific literature.

\subsubsection{Non-indigenous species in Mission Bay}

Musculista senhousia

Among the most harmful non-indigenous species are those that outcompete native species to the point of eradication from an area (Crooks 2001). The Asian mussel, Musculista senhousia, is an example of an NIS that has shown the ability to dominate an ecosystem. Between the 1960's and the 1990's, M. senhousia densities in Mission Bay increased from relatively low values to a population over 100 times as abundant as any native species in subtidal areas of the bay (Crooks 2001). In 1996, M. senhousia accounted for over $90 \%$ of the bivalve biomass in samples collected from Mission Bay (Crooks 2001). M. senhousia was generally observed in greater densities toward the back of the bay by Dexter and Crooks (2000), who speculated that this distribution followed the pattern of exotic species favoring low habitat quality (Elton 1958). M. senhousia has been shown to have negative impacts on survival and growth rates when placed with suspension-feeding native bivalve species (Crooks 1998a, 2001). This effect is the result of its communal living structure and ability to modify its environment (Crooks 1998a). M. senhousia tend to group together and alter their environment through the formation of byssal mats, which are a combination of byssal threads and sediment (Crooks 1998a). A decrease in species diversity among native species of bivalves, such as Solen rostiformis, was observed in Mission Bay, coinciding with the rise in M. senhousia abundance (Dexter and Crooks 2000, Crooks 2001). While there was a 
decrease in the abundance of other suspension-feeding bivalves, densities of specific tanaid and gastropod species were enhanced within the byssal mats of $M$. senhousia (Crooks 1998a).

Venerupis philippinarum

Commonly known as the Manila clam or Japanese littleneck clam, Venerupis philippinarum is native to Asia but was introduced to the northern Pacific coast of North America in the mid-1930's as a side-effect of the introduction of the Japanese oyster (Carlton 1979, Burnaford et al. 2011). V. philippinarum is thought to have reached southern California in the 1990's (Burnaford et al. 2011) and has recently been collected in Mission Bay (Novoa et al. 2016.). Studies have indicated that $V$. philippinarum does not have a strong negative effect on species richness or abundance of other bivalves, but it tends to occur in very high densities (Burnaford et al. 2011) and is able to establish populations very quickly. In Colorado Lagoon (southern California), V. philippinarum was unknown before 2000 but accounted for $87.6 \%$ of the bivalve population after a period of rapid growth between 2000 and 2003 (Burnaford et al. 2011). Byers (2005) found that even at densities $50 \%$ higher than those observed in the field, V.philippinarum did not have a direct effect on the survival of the native Protothaca staminea. In fact, V. philippinarum may protect native species from predation and was shown to be the preferred food source for predatory crabs in reserves around the San Juan Islands in Washington (Byers 2005). Due to the high abundance of $V$. philippinarum, its presence may facilitate large increases in the biomass of predator species, therefore increasing the overall number of predators in the system and 
potentially leading to a decrease in population of native prey species (Byers 2005). In the context of this experiment Venerupis may be a better basis of comparison than Musculista for Chione in terms of size, physiology, and behavior, as they are both clams. Musculista is a mussel and has been shown to have faster growth rates, live in communities and bind to surfaces using their byssal threads, which makes them quite different from Chione (Crooks 1998a).

\subsubsection{Copper as an environmental toxin}

Copper is a common pollutant in bays and harbors (Neira et al. 2009). Copper enters the water through several sources, including from antifouling paints on boats, wood preservatives, industrial runoff, highway runoff, and sewage waste (Zirino et al. 1998, Piola and Johnston 2008). Antifouling paint on recreational boats has been found to leach copper into aquatic environments (Schiff et al. 2004). In a recent study of Shelter Island Yacht Basin in San Diego Bay, copper from antifouling boat paint accounted for $92 \%$ of the total dissolved copper in the system (Neira et al. 2009). Copper can exist in several forms within the water column, including dissolved and ligand-bound copper (Zirino et al. 1998), and has functional uses within the body, such as being a cofactor in many enzymes (Salviati et al. 2002). Dissolved copper is the most harmful form of copper to suspension-feeding bivalves, due to the direct method of exposure to soft tissue during filtration, e.g. at exchange sites such as the gills (Strom et al., 2011). More specifically, free copper $\left(\mathrm{Cu}^{2+}\right)$ is viewed as the most harmful form for organisms (Zirino et al. 1998, Strom et al. 2011, Vosloo et al. 2012). This is because $\mathrm{Cu}^{2+}$ is able to bind to the tissue of the organism itself and cause physiological damage, 
including destruction of gas exchange surfaces such as gills (Gregory et al. 2002). In fishes, free copper has been shown to bind directly to the gills (Taylor et al. 2003). In the water column, the majority of dissolved copper is bound to organic material. Dissolved copper accumulates within suspension-feeding bivalves through filtration of organic material (Anandraj et al. 2002). Some of the observed effects of dissolved and accumulated copper include increases in rates of mucus secretion and oxygen uptake, and loss of DNA integrity (Vosloo et al. 2012).

As an environmental toxin, copper has been shown in many studies to have a clear and significant negative effect on both populations and the health of marine organisms (e.g., Piola and Johnston 2008, 2009, Piola et al. 2009, Crooks et al. 2011, AlSubiai et al. 2011). This negative effect may be sublethal but often leads to mortality. A negative correlation between copper exposure and survivorship of test subjects has been reported in numerous studies, several involving bivalve species in particular (Sunila 1981, Sunila and Lindstrom 1985, Grout and Levings 2001, Anandraj et al. 2002, AlSubiai et al. 2011). Copper often enters the system of a bivalve through two pathways: respiration and food consumption (Anandraj et al. 2002, Turner et al. 2009, Al-Subiai et al. 2011, Vosloo et al. 2012). Furthermore, copper has been shown to have far reaching impacts beyond those on established adult bivalves, as it has been observed to have a negative impact on both the survival and the ability of larvae to settle in coppercontaminated environments (Reichelt-Brushett and Harrison, 2000).

Sediment is a known repository for copper in the aquatic environment, as it serves as a copper sink, and has had an impact on some species of bivalves, However, studies have shown that exposure to copper in the water column is much more toxic 
and negatively affects the fitness of suspension-feeding bivalves more than exposure to copper through the sediment, even though many suspension-feeding bivalves burrow or cover themselves in sediment (Absil et al. 1996). It has been postulated that the shell of the organism is an effective barrier to copper, and that physical contact between copper and shell is not an effective pathway for copper into the body. In contrast, the feeding habits of the organisms have a greater effect on copper accumulation, as shown by the difference reported by Absil et al. (1996) between the suspension-feeding bivalve Cerastoderma edule and the deposit-feeding bivalve Macoma balthica, which comes into contact with the sediment when feeding. Cerastoderma was influenced more by copper levels in the water, and Macoma was affected more by sediment copper levels.

The fact that copper concentrations in the water column have been shown to correlate with copper accumulation in prior studies indicates that ingestion or respiration, two processes based on intake of surrounding water into the body, are the main routes of copper exposure (Anandraj et al. 2002, Turner et al. 2009, Al-Subiai et al. 2011, Vosloo et al. 2012). In other studies examining sublethal effects, particular attention has been paid to the gills and aspects of the digestive tract and the processes that involve the two, including filtration rate and oxygen consumption (Anandraj et al. 2002, Al-Subiai et al. 2011, Vosloo et al. 2012). A study examining bivalve copper exposure from boat paints showed a clear depletion of copper in the surrounding water column during periods of observed feeding, which indicated that feeding is a main mechanism through which copper enters the body in bivalves (Turner et al. 2009). 


\subsubsection{Copper chemical speciation in water column and free copper $\left(\mathrm{Cu}^{2+}\right)$}

Copper has been observed throughout the water column. It is found in dissolved forms in surface and bottom water, as well as in sediment porewater. Dissolved copper can take two forms: complexed dissolved copper, which is copper that has bound to organic material in solution, and unbound or "free" copper, which is designated as $\mathrm{Cu}^{2+}$ (Neira et al. 2009). An estimated $99.9 \%$ of dissolved copper in coastal marine environments is complexed by organic material, rendering it less dangerous to organisms than unbound copper (Zirino 1998, Buck et al. 2005). Although total dissolved copper may be a good indicator of toxicity (Zirino et al. 1998), free copper has been determined to be the best indicator of copper toxicity (Sunda et al. 1998), and is generally considered to be the most harmful form of copper to organisms (Sunda et al. 1998, Neira et al. 2009). Because $\mathrm{Cu}^{2+}$ is a dissolved ion, suspension-feeding bivalves can be exposed to free copper through their filtration and gas exchange systems, a more direct route of exposure than some other forms of copper, such as sediment copper (Neira et al. 2009).

\subsubsection{Biological effects of copper}

Effects that have been observed in different species from copper-contaminated ecosystems include: impaired reaction to predation; reduced colonization and burrowing; reduced feeding rate and survivorship; impaired habitat selection, effects on fertilization, embryonic development and chemosensation; and inhibition of larval settlement (Rygg 1985, Trannum et al. 2004, Pyle and Mirza 2007). Altered respiration rates, lower feeding rates and lower growth rates have been accepted as indicators of 
organism condition and physiological efficiency when placed under environmental stress such as the presence of pollutants (Widdows 1985, Sobral and Widdows 1987). Dissolved copper concentrations ranging from 18-56 parts per billion ( $\mathrm{ppb}$, or $\mu \mathrm{g} \mathrm{L}^{-1}$ ) have been shown to have a negative impact on the suspension-feeding bivalve, Mytilus edulis, based on physiological damage (Al-Subiai et al. 2011). Concentrations of 18, 32, and $56 \mathrm{ppb}$ copper produced a significant decrease in filtration rates, and concentrations of 32 and 56 ppb copper significantly increased concentrations of glutathione in the gills and digestive glands. Copper concentrations in the adductor muscle, digestive gland, and gills also were higher in all experimental groups exposed to copper, compared to the control (Al-Subiai et al. 2011).

Mucus secretion rate is another health indicator that has been examined in suspension-feeding bivalves. Mucus secretion is considered to be a biological response to metal exposure (Anandraj et al. 2002, Vosloo et al. 2012). Mucus secretion has been established as a protection method for bivalves exposed to metals, as mucus can be used to sequester the metals, compensate for gill damage, and prevent re-exposure (Sze and Lee 1995). Exposure to concentrations of 25 and $50 \mathrm{ppb}$ dissolved copper produced a statistically significant increase in mucus secretion in specimens of the filter feeding bivalve Perna perna, indicating that the organisms may have been attempting to prevent physiological damage from copper exposure (Vosloo et al. 2012). 
1.1.10 How this project addresses the effects of copper on non-indigenous and native species

This study is designed to compare copper tolerances of non-indigenous and native species of suspension-feeding bivalves that are commonly found in Mission Bay to examine the potential for a competitive advantage in a copper-contaminated environment. Specifically, I propose to examine how metabolism (as reflected in filtration rates) and growth rates of non-indigenous bivalves differ from those of native species exposed to elevated concentrations of copper. Initial growth rates can be compared to post-exposure growth rates and compared across species. Examination of how mucus secretion rates differ between NIS and native species in response to various copper concentrations will also be used as a marker of toxicity. These sublethal measures are useful for this study, because copper concentrations in water samples taken from Mission Bay in 2002 showed a moderate level of copper contamination, with maximum concentrations of 26.6 ppb near the mouth of Tecolote Creek in May 2002 (Kaufmann et al. 2004). In research involving different species, these concentrations were considered to be nonlethal based on the fact that Anandraj et al. (2002) and Vosloo et al. (2012) were able to use concentrations of dissolved copper up to $50 \mathrm{ppb}$ to examine sublethal effects on a similar bivalve species, Perna perna, and Mytilus edulis, which were exposed to concentrations as high as $56 \mathrm{ppb}$ with no mortality (Al-Subiai et al. 2011). These studies support the results of a pilot study examining survivorship at high copper concentrations, where the species used in this experiment were exposed to different copper concentrations and were observed as having survived at concentrations as high as $50 \mathrm{ppb}$ (Table 2.1). 
To examine if copper contamination has significantly different effects on NIS and native species, this project includes two non-indigenous species and one native species of suspension-feeding bivalves found in Mission Bay. The use of a suite of species has been effective in showing trends in the environmental tolerance mechanisms of non-indigenous species with regard to contaminants (Crooks et al. 2011). A suite of species is good for showing trends across species and/or taxa by providing a larger picture of the impact of a given variable. As a group, many nonindigenous species of marine invertebrates have been shown to be more tolerant of copper than native species, with non-indigenous species richness being largely unaffected in the face of increasing copper concentrations while the species richness of native species was negatively affected (Crooks et al. 2011). This indicates that the reactions to copper could vary among the species that will be used in this research. By studying several native and non-indigenous species, this research examines whether the trend of non-indigenous species having a higher tolerance to sublethal concentrations of copper is supported for the suspension-feeding bivalve species in Mission Bay. 


\section{Chapter 2: The Impact of Copper on Non-Indigenous and Native Species of Suspension- Feeding Bivalves in Mission Bay, San Diego, California}

\subsection{Introduction}

Non-indigenous species (NIS) are considered to be a threat to ecosystems, in part due to their adverse effects on populations of native species. However, not all interactions between native and non-indigenous species are the same, nor are the ways that they react to external pressures (Lenz et al. 2005). Copper, a pollutant found in anti-fouling paints commonly used on boat hulls, has been shown to have negative impacts on many types of aquatic organisms, beyond those that cause fouling (Bracey, 1994). There is evidence that, when compared side by side, some NIS have higher copper tolerances than similar native species (Crooks et al. 2011). Bivalves have been common research subjects for testing the effects of heavy metals like copper on aquatic life, and several mechanisms in particular have been singled out for study to examine the impact of copper. Past studies have indicated that processes such as mucus production and food filtration have been impacted by the presence of copper in test bivalve species, and that growth in some cases has been stunted by copper exposure. This research has mostly been done on common research organisms such as Mytilus galloprovincialis and M. edulis, and not on species local to Southern California. Research has also been scarce on comparisons between NIS bivalves and comparable native species in terms of reaction to copper exposure, and this study proposes a more focused examination of the impact of copper on bivalve species, with a comparison between native species and NIS. 
Mission Bay is an ecosystem heavily impacted by anthropogenic activity and known to host a number of non-indigenous species of suspension-feeding bivalves. Populations of the non-indigenous species Musculista senhousia in Mission Bay expanded rapidly between the 1960's and 1990's, increasing from a relatively small population to 100 times more abundant than any native bivalve species in some areas of the bay (Crooks 2001). Native species such as Chione fluctifraga and Chione undatella have been negatively impacted by the presence of Musculista senhousia (Crooks 2001). One factor that may make Mission Bay more vulnerable to invasion is pollution. Copper, a pollutant found in anti-fouling paints commonly used on boats, is a prominent toxin in Mission Bay (Kaufmann et al. 2004). This study will examine how copper concentrations impact biological processes in non-indigenous and native suspension-feeding bivalve species in Mission Bay. Through the examination of these sublethal effects, the influence of environmental copper can be evaluated as a factor affecting competition between non-indigenous and native bivalve species.

The examination of physiological responses by non-indigenous and native bivalve species to a common pollutant such as copper can give insight into one of the factors that impacts success in competition for resources. Examining multiple NIS and native species should contribute to identifying larger trends in the nature of invasions and may illustrate what makes some species more successful than others in contaminated locations. The presence of NIS and a gradient in copper concentrations make Mission Bay an ideal site for collection of specimens to examine the effects of copper on bivalves. By examining the impact of anthropogenically-influenced copper contamination on the biological processes of suspension-feeding bivalves, this research provides a closer look at whether copper contamination can alter the competition for 
resources in the suspension-feeding bivalve community and can be used as an indication of human impact on non-indigenous/native species competition in Mission Bay.

The focus of my research can be split into two categories: the effects of different copper exposure levels on bivalve species, and the comparison of these observed effects between native and non-indigenous bivalves. The purpose of this study is to gather and report information regarding the ability of local bivalve species to survive copper exposure, and the impact that copper has on some biological processes. In addition, this study is intended to highlight what, if any, differences in tolerance to copper can be observed between the NIS and native species and suggest future success of each in the event of increased copper pollution to the environment. Through examination of how local species react to copper exposure, this study will add to the knowledge base of copper impact on bivalve species by looking at uncommonly studied species, and will also provide more information on how non-indigenous species perform under copper exposure in relation to comparable native species.

\subsection{Methods}

\subsubsection{Sample Collection}

Mission Bay, a small, extensively modified mesotidal estuary in San Diego, California, was the site of sample collection. The primary collection site was a small, isolated saltwater pond located on Vacation Island, in the center of Mission Bay. In this area, populations of each species were sufficient to easily sustain multiple collections of over 50 specimens per species. 
Three species of bivalve were collected during the spring and summer in 2014: Musculista senhousia, Chione californiensis, and Venerupis philippinarum. There were three separate collection events: 4/14/2014 (a pilot study), 5/31/2014, and 7/21/2014, from which 50 adult specimens of each species were collected for each phase of the experiment. Specimens for the second and third collection periods were selected based on size, with an emphasis on ensuring that within each species there was a consistent size range for each collection. Water samples were taken for copper analysis to establish total copper concentration at the collection site. Immediately after collection, specimens were taken to the University of San Diego and placed in a temperature controlled cold room set at $17.5^{\circ} \mathrm{C}\left(63.5^{\circ} \mathrm{F}\right)$ to mimic temperature conditions commonly found in Mission Bay (Kaufmann et al. 2004). These bivalves were left in the cooler they were collected in with water from the pond to acclimate for 24 hours, and then were placed in depuration tanks of filtered seawater to allow for release of any environmental toxins. Bivalves remained in this tank for a period of one week, with one $50 \%$ water change conducted after three days.

\subsubsection{Experiment design, depuration, and tank setup}

For each collection conducted, there was a subsequent experimental period following the depuration process. The 4/14/2014 collection had an experimental period lasting 24 days, the 5/31/2014 collection had an experimental period that lasted 18 days, and the $7 / 21 / 2014$ collection had an experimental period that lasted 12 days. The depuration tanks were filled with filtered seawater and were designed to allow the bivalves to release any residual toxins they may have accumulated during their time in 
Mission Bay. A total of three depuration tanks were used: one depuration tank for each species. The depuration tanks underwent a single $100 \%$ water change after three days to eliminate any toxins that had been released by the bivalves. This was done by preparing new depuration tanks the day prior and allowing these to equilibrate in the cold room to reach the same temperature as the initial tanks, thus reducing temperature shock to the animals.

After the depuration process had concluded, a series of tanks was set up in the temperature controlled room in a randomized fashion to eliminate as much environmental variation from tank location as possible. For each of the experimental periods there were a total of nine $48 \mathrm{~L}$ experimental tanks set up with different seawater total dissolved copper concentrations, with three replicates for each concentration. The control tanks for each experiment consisted of $24 \mathrm{~L}$ of filtered seawater, with no added dissolved copper. Experimental tanks with added dissolved copper were also set up, with three tanks calculated to have a dissolved copper concentration of $25 \mathrm{ppb}$, and three tanks calculated to have $50 \mathrm{ppb}$. During the pilot study, there was also a single experimental tank with a calculated dissolved copper concentration of $100 \mathrm{ppb}$ set up to establish toxicity limits for the bivalve species.

Following the depuration period, specimens were labeled to differentiate individuals from one another. This was achieved by marking each individual with three dots of nail polish, with color combinations selected from four different colors. Shell size was measured using calipers at the beginning and end of the experiment, to estimate growth rates for each individual that survived the entire experiment. Each individual was marked and then measured using a set of digital calipers to the nearest $0.01 \mathrm{~mm}$ 
across the anterior-posterior axis for the clam species, and along the ventral-dorsal axis for the mussel species. These values were recorded for later use in growth analysis.

All experimental tanks were dosed with copper using a $5 \mathrm{mg} \mathrm{L}^{-1}$ solution of cupric sulfate pentahydrate in distilled water. Due to the volume of the tanks and the volume of stock solution added, it is unlikely that the addition of distilled water copper stock affected salinity. $50 \%$ water changes were conducted using clean filtered seawater stored in the temperature controlled room to ensure minimal temperature variation during the water change. Water samples were taken both immediately before the initiation of the experiment and immediately before the water change. These samples were acidified using $125 \mu \mathrm{L}$ of quartz-still grade nitric acid, and later quantified with a Perkin-Elmer SCIEX ELAN DRCII ICP-MS to establish copper concentration. Water changes were dosed with the appropriate amount of $5 \mathrm{mg} \mathrm{L}^{-1}$ copper solution to achieve the specific dissolved copper concentrations within the tanks. Water changes were scheduled every 6 days. Air stones were placed in each tank to promote water flow and oxygenation of the water.

Five specimens of each species were placed in each tank, for a total of 45 specimens of each species for each experimental period. An additional tank calculated to have $100 \mathrm{ppb}$ of dissolved copper was set up with 50 specimens of each species during the initial experiment. The goal was for all specimens to be subject to the same environmental conditions within the tank while keeping the species separate and unable to interact with one another. To achieve this, Chione and Venerupis specimens were placed on opposite sides of the aquarium and a short acrylic block was placed between them, effectively isolating the species from one another, while minimally impeding 
water flow. Musculista specimens were placed in small plastic baskets fitted with plastic lids. These baskets floated at the surface but were deep enough to submerge all Musculista specimens to half of the aquarium's depth and isolate the Musculista from the other two species.

Initiation of the exposure groups was staggered for maximum efficiency. Since some processes required multiple hours for one exposure group, it was necessary to allow one day for working with each exposure group. The groups in tanks with no copper added were initiated first, followed by the groups in tanks calculated to have 25 $\mathrm{ppb}$ and then $50 \mathrm{ppb}$ of copper. By the time the final group was initiated, the initial group was on day 2 , with the first algae depletion measurement beginning the next day. This setup allowed for the most efficient use of time, as there would be an algae depletion measurement occurring each day for the duration of the experiment (Appendix B, Table 1.1).

Individual bivalves were fed on a schedule that accommodated the algae depletion experiments over the course of the experimental period. Each treatment group was subjected to an algae depletion experiment once every three days. To ensure that specimens used in the experiments were starved ahead of time, feedings of each treatment group coincided with the dates that each treatment group was being tested for algae depletion, after the test specimens were removed. These test specimens were fed individually to measure algae depletion, ensuring that all specimens were fed similarly for the duration of the experiment. For the second and third experimental periods, each tank was fed $100 \mathrm{~mL}$ of a highly-concentrated Nannochloropsis oculata suspension. These phytoplankton typically range from 2 to $6 \mu \mathrm{m}$ in diameter. A pilot 
study was fed Isochrysis galbana, but availability concerns led to a switch to $N$. oculata. Due to this, algae depletion data for that pilot study were not used for the data analysis comparison between experiments.

\subsubsection{Algae Depletion Setup and Procedure}

During the course of the experimental exposure periods, two main data sets were accumulated: bivalve survivorship and algae depletion. Algae depletion was tested on a rotating basis by exposure group. Each day, a depletion run would be completed for one exposure group, and a depletion run would be initiated for the next exposure group (Appendix B, Table 1.1). An initial pilot study was done to determine if algae depletion was occurring through suspension feeding or if algae removed from solution were merely collecting on the shells of the bivalves. Live bivalve specimens were placed in plastic beakers with Nannochloropsis on a platform with a spin bar and left for 24 hours. A control of an empty beaker was used, and a second control of a beaker using only an empty shell was also used. It was shown that there was no statistical difference between control beakers and those with empty shells, indicating that depletion was due to intake of algae and not removal by the shells themselves. However, there was significant depletion of algal cells in the beakers with live bivalves. There was a procedural difference between the 18 day and 12 day exposures: the number of subsamples collected at each time point was decreased from three to two in an effort to conserve diluent.

The procedure for algae depletion was tested in pilot studies to determine the optimum concentration of algae used and duration for feeding. After several tests, it 
was shown that 24 hours of exposure to algae showed greater depletion than shorter durations. Exposure periods of 6 and 12 hours did not show similar levels of algae depletion, while increasing the time period to 36 hours did not show a significant difference from the 24 hour exposure. Specimens being tested for algae depletion were selected at random to eliminate bias. After the pilot study, it was determined that a mix of $60 \mathrm{~mL}$ of seawater and $40 \mathrm{~mL}$ of Nannochloropsis algae solution was best in terms of conserving resources and showing measurable depletion rates. At the initiation of the experiment, the mixtures of Nannochloropsis and seawater were placed into a series of eight $150 \mathrm{~mL}$ plastic beakers with small magnetic stir bars. Beakers were placed on magnetic stir plates, with two beakers for each group: control (no specimen), Chione, Musculista, and Venerupis. Small platforms were crafted using a section of PVC tube, plastic mesh, and zip ties and placed into each beaker over the stir bars, with holes drilled in the side of the tube to allow water flow (Figure 1.1). Specimens were then placed on the center of these platforms, exposing them to continuously mixed solution without coming into contact with the stir bars. The control beakers had the same setup of platform with stir bar, and were presented with the same concentration of algae, but lacked live bivalves to deplete the algae.

Specimens were placed in the solution with the stir plates activated for a period of 24 hours, and water samples were taken for measurement of initial cell density during each algae exposure period. In each case, the initial water sample was taken immediately prior to the specimens being placed in the algae solution. At the conclusion of the 24 hour period, specimens were removed and returned to their respective tanks, and water samples were taken to determine algal cell depletion. In each instance, $10 \mathrm{~mL}$ of the sample was mixed with $10 \mathrm{~mL}$ of Isoton II diluent, and then run through a 
Figure 1.1: Diagram of the experimental design and setup of the algae depletion experiment

$60 \mathrm{~mL}$ filtered seawater, $40 \mathrm{~mL}$ Nannochloropsis

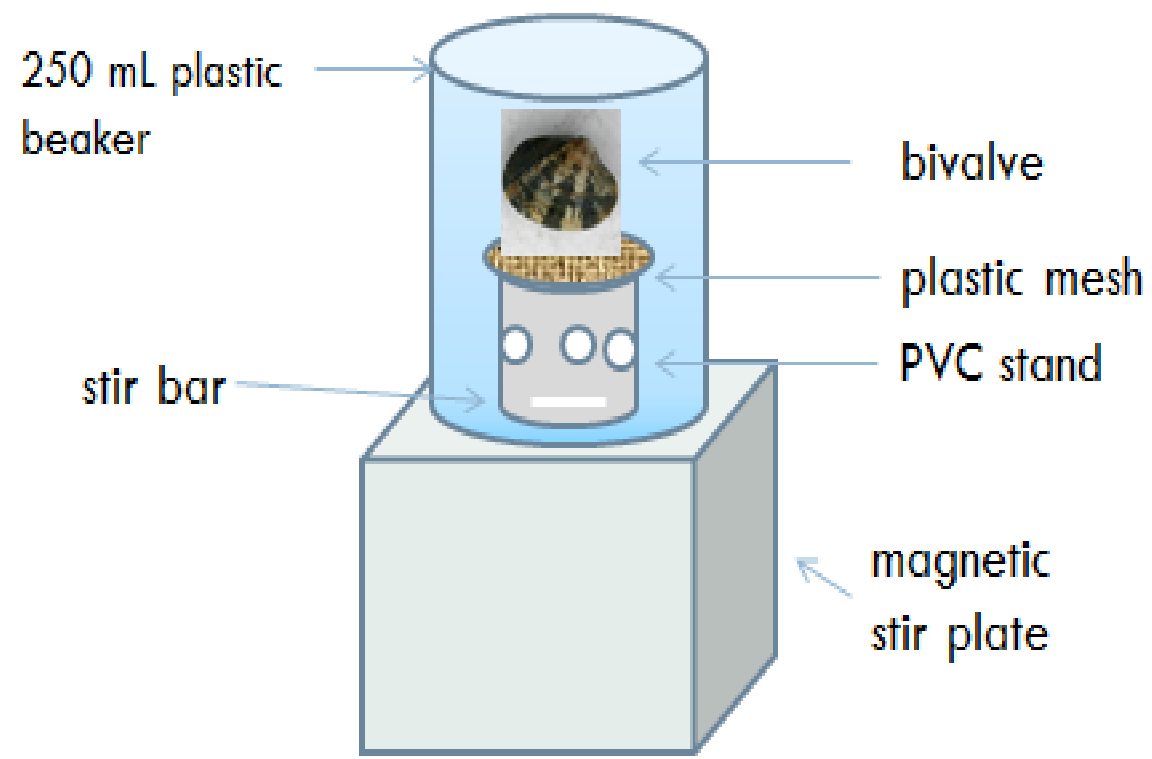

Algae depletion experimental setup 
Multisizer 3 Coulter Counter using a glass tube with an aperture of $50 \mu \mathrm{m}$, allowing for the measurement of the Nannochloropsis cells. After measuring each sample, three times (or twice, in the case of the 12 day exposure), a difference was calculated between the initial cell density and cell density of samples taken at the end of exposure, to determine the algal cell depletion rate.

\subsubsection{Survivorship and post-experiment growth measurement}

Survivorship was checked at least once per day. Specimens that were clearly dead, as determined by open shells and, in some cases, cloudy water, were removed immediately, while specimens that appeared unhealthy were noted and later rechecked. Unhealthy organisms were identified by slow or incomplete closure of the shell when faced with stimuli; healthy bivalves typically closed their shells quickly at any disturbance. All mortalities were recorded and logged, noting which individual had expired and the date of expiration. Any decomposing tissue in the tank was removed using a fish net.

At the conclusion of the experimental periods, shell widths of all surviving specimens were measured using digital calipers. Survivors then were divided evenly into two groups when possible. If an odd number survived, the extra specimen was used for mucus collection to increase the sample size for that measure. Half of the specimens were designated for mucus collection, while the other half of the specimens were frozen immediately after the experiment and thawed at a later date for dissection in order to obtain the gills and digestive tract. 


\subsubsection{Mucus collection}

From examination of previous studies that have used mucus production as a tool in analyzing the effects of copper on bivalves, the established metric is the ratio of mucus to dry mass, which is the mucus produced by the bivalve after the exposure period compared to the dry mass of the bivalve (Sze and Lee 1995) in order to correct for differences in size. Surviving bivalves designated for mucus collection were gently pried open using a razor blade, and a glass slide (for Chione and Venerupis) or cover slip (for Musculista) was placed in the shell opening. The bivalve was then held in place by wedging the slide into a plastic beaker and inverting the bivalve for a period of 30 minutes to allow for mucus collection on the slide.

After 30 minutes passed, the slide or cover slip was removed, the accumulated mucus was rinsed into a pre-weighed weigh boat, and the boat was placed into a $60{ }^{\circ} \mathrm{C}$ oven for 24 hours. The bivalves were then cut from their shells, placed in pre-weighed weigh boats, and the boats placed into the oven for 24 hours. After 24 hours, samples were removed, weighed, and the dry weight of both mucus and bivalve tissue calculated and applied to the ratio of mucus/dry mass. Copper in the dry bivalve tissue was analyzed to generate a value for whole body copper accumulation.

\subsubsection{Tissue Copper: dissection, digestion, and analysis}

Specimens were dissected to obtain gill and digestive tract tissue, as these particular organs are associated with exposure to contamination from water and particulate matter, respectively (Turner et al. 2009, Al-Subiai et al. 2011). These organs 
were digested individually to measure copper accumulation. After dissection, tissue samples were placed in pre-weighed plastic weigh boats and dried in an oven at $60^{\circ} \mathrm{C}$ for a minimum of 24 hours to remove all water from the tissue. Once dried, the specimens were weighed to determine their dry mass. Specimens were then digested following the standard guidelines for the EPA 3052 (EPA 1996) protocol of microwave tissue digestion, with some modifications in volume and substituting nitric acid in the place of hydrofluoric acid, due to availability. Dried specimens were digested in Teflon containers using $6 \mathrm{~mL}$ of $12 \mathrm{M}$ nitric acid, $2 \mathrm{~mL}$ of hydrogen peroxide, and $3 \mathrm{~mL}$ of MilliQ water, which yielded approximately $10 \mathrm{~mL}$ of digestate. The digestate was transferred to a $50 \mathrm{~mL}$ centrifuge tube and diluted with $40 \mathrm{~mL}$ of Milli-Q water. Copper in this diluted sample was quantified with a Perkin-Elmer SCIEX ELAN DRCII ICP-MS, and copper per gram dry mass was calculated from these data.

\subsubsection{Statistical Methods}

Most statistics were run using R. The Shapiro-Wilks normality test was used to determine normality and determine whether the data should be analyzed using parametric or nonparametric tests.

In order to determine if there were statistical differences between species and between copper concentrations in relation to survivorship, copper accumulation, growth, and algae depletion, data sets were run through factorial two-way ANOVA testing with interaction terms if the distribution was normal and through a KruskalWallis test if nonparametric tests were appropriate, using a parameter of $p=0.05$ as an indicator of statistical significance. These tests were run to examine significant 
differences between copper concentrations and significant differences between species. If significant differences were found in any analysis of variance, a post hoc Tukey's HSD test was used to establish which treatment was different from the others, for the ANOVA tests, and a Dunn's test with Bonferroni correction was used for post hoc testing of results from the Kruskal-Wallis tests.

Data sets were initially separated between experiments, as the exposure periods for the two experiments were different durations. In addition, the data were further broken into several different groups based on the information they contained. Data that were grouped together were: Survivorship, Mucus Production, Algae Depletion and Feeding Rate, Growth, and Tissue Copper. Some of these groups were compared against each other in regressions to examine if they were significantly correlated. Statistics relying on percentages, such as survivorship rates and growth percentages, were first transformed into arcsine values for statistical analysis. In several instances, a log transformation was required to normalize a data set.

\subsection{Results}

\subsubsection{Pilot Study}

Prior to the main experiment, a pilot study was designed and conducted to determine the lethality of several concentrations of copper. The purpose of the pilot study was to establish the parameters of the main experiment in relation to the copper concentrations targeted for the Medium and High copper exposures. Specimens were exposed to calculated copper concentrations of $0 \mathrm{ppb}, 25 \mathrm{ppb}, 50 \mathrm{ppb}$, and $100 \mathrm{ppb}$ to 
examine survivorship rates over a period of 24 days, with three tanks for each of the 0 , 25 , and $50 \mathrm{ppb}$ exposures, and a single tank for $100 \mathrm{ppb}$. The goal of the pilot study was to determine which concentration would serve as a High copper exposure for the main experiment, with a focus on high lethality without a mortality of $100 \%$, so that sublethal factors could be tested for specimens exposed to the High copper exposure.

Survivorship patterns from the pilot study indicated that Chione had higher survival rates than the other two species at high copper concentrations, as Chione had a survivorship percentage of $53.33 \%$ at $50 \mathrm{ppb}$ in comparison to the $6.67 \%$ survivorship of Musculista and $26.67 \%$ survivorship of Venerupis. Chione was also the only species with a specimen that survived in the $100 \mathrm{ppb}$ tanks (Table 8.1). The results of the pilot study indicated that copper concentrations at $100 \mathrm{ppb}$ are lethal to the organisms tested, while there were higher levels of survivorship at $50 \mathrm{ppb}$ for all three species (Table 8.1). As a result, $50 \mathrm{ppb}$ was selected as the maximum concentration target for the main experiment.

\subsubsection{Water Copper Concentration}

Copper concentrations in the test tanks were measured by ICP-MS. As no copper was added to Control tanks, they were expected to have the copper concentration of the source water. Experimental tanks were expected to have concentrations of about 25 and 50 ppb. Measured mean \pm one standard deviation copper concentrations for both experiments were $6.50 \pm 1.11 \mathrm{ppb}$ for the tanks to which no copper was added $(\mathrm{N}=5), 23.1 \pm 4.27 \mathrm{ppb}$ for the "25 ppb" tanks $(\mathrm{N}=5)$, and $44.1 \pm$ $4.29 \mathrm{ppb}$ for the "50 ppb" tanks ( $\mathrm{N}=6$ ) (Fig. 1.2). Since measured copper concentrations 
in these tanks were not exactly 0, 25 and $50 \mathrm{ppb}$, the three dosing levels were labeled as Low, Medium and High copper treatments.

A Shapiro-Wilk test showed that the distribution of measured copper concentrations was normal $(p=0.051)$, and running an ANOVA revealed statistically significant differences between the groups $\left(p=1.05 \times 10^{-9}\right)$. A Tukey's HSD post hoc test further showed that the dissolved copper measurements in the Low, Medium and High copper treatment groups were statistically different from each other $(p \leq 0.001$ in each comparison). The results of the Tukey's HSD post hoc test show that differences between measured copper concentrations were large enough that the individuals in various treatments were exposed to different copper concentrations (Figure 1.2). The initial copper concentrations of each dosage range tank (Low, Medium, High) were pooled to increase the $\mathrm{n}$ value and provide more statistical power.

When examining the values further, there appeared to be a disparity between the initial concentrations of copper in the 18 day and 12 day exposures. The 18 day exposure copper concentrations were lower than the 12 day exposure concentrations for each treatment group, with the greatest difference $(6.7 \mathrm{ppb})$ occurring in the High copper group (Fig. 1.3, Fig. 1.4A). Dissolved copper concentration was tested after the water change that occurred on day 6 of each experiment to examine changes in dissolved copper concentration during the experiment.

Copper concentrations in the water did not follow a consistent decreasing or increasing trend during each experiment. In the 12 day experiment, the Medium copper tanks copper concentration on day 6 was less than on day 0 , by almost $5 \mathrm{ppb}$ in each instance (Figure 1.4B). This pattern was also true of the High copper tanks during the 18 
day experiment; these showed a drop of $11 \mathrm{ppb}$. However, the Medium copper tanks in the 18 day experiment showed slight increases in copper concentration at the time of the water change (Appendix B, Table 1.1, Figure 1.4B). It should be noted that some of the copper concentrations from the Low copper group were also higher at the water change than they were initially, for both the 18 day and 12 day exposures (Appendix B, Table 1.1). 
Figure 1.2. Estimated vs. measured copper concentrations across both experiments. Measured $\mathrm{Cu}$ values represent a mean across tanks for each experiment ( $\mathrm{N}=3$ for each mean). Error bars represent \pm one standard deviation.

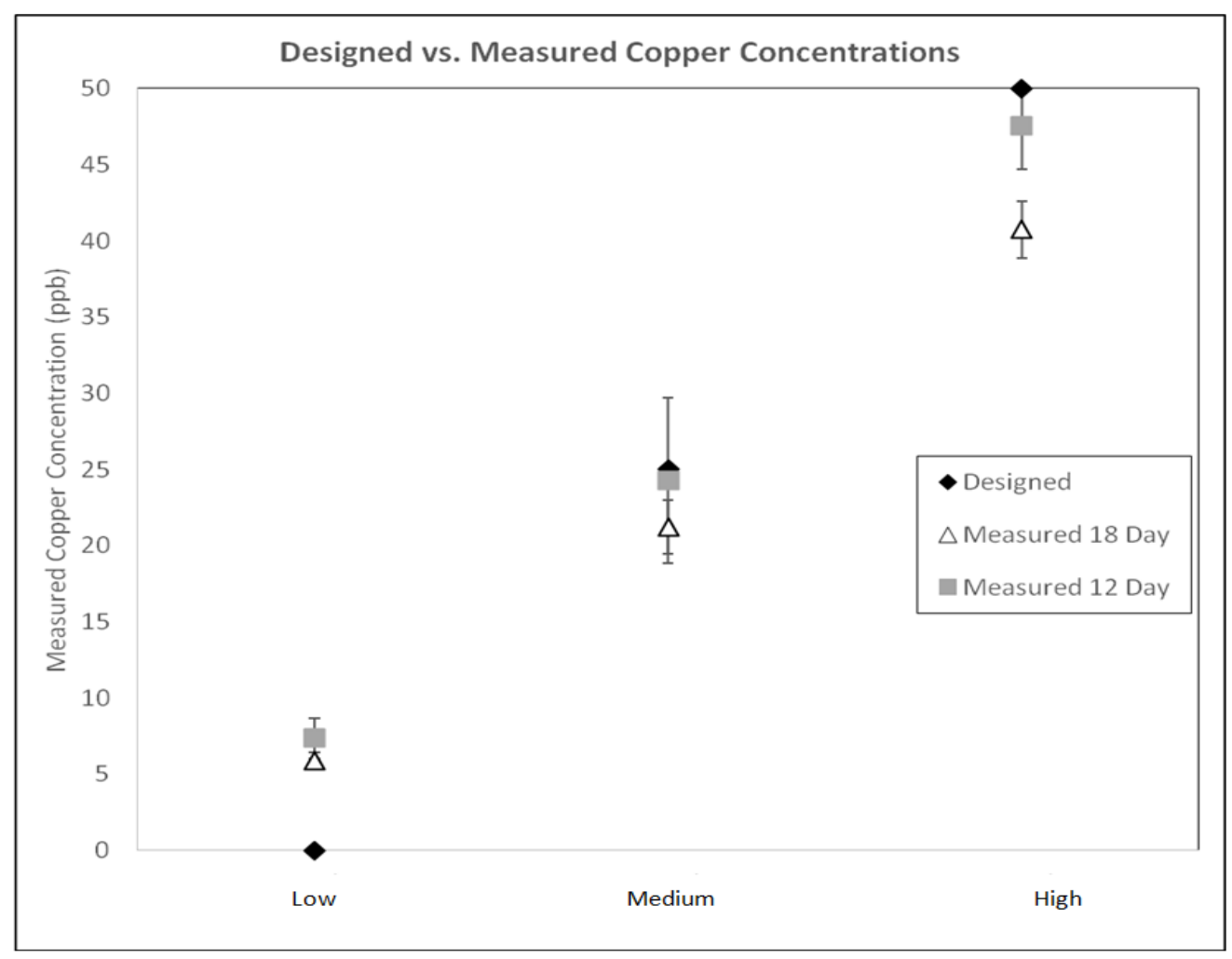


Figure 1.3 Visual representation of randomized experimental layout. This figure shows estimated copper concentrations, initial number of specimens, water change time, initial measured copper concentrations, and copper concentrations on Day 6. Initial copper concentrations were not measured for the Medium copper tank 4 for the 18 day experiment and the Low copper tank 1 for the 12 day experiment.

A.) Tank layout and information for 18 day experiment

B.) Tank layout and information for 12 day experiment 


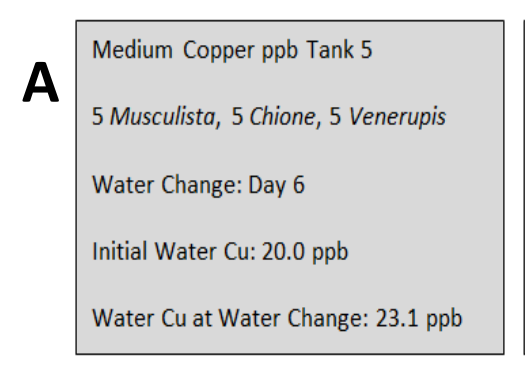

Low Copper Tank 1

5 Musculista, 5 Chione, 5 Venerupis

Water Change: Day 6

Initial Water Cu: 5.4 ppb

Water $\mathrm{Cu}$ at Water Change: $7.5 \mathrm{ppb}$

Medium Copper Tank 6

5 Musculista, 5 Chione, 5 Venerupis

Water Change: Day 6

Initial Water Cu: $22.5 \mathrm{ppb}$

Water Cu at Water Change: $21.1 \mathrm{ppb}$
Low Copper Tank 2

5 Musculista, 5 Chione, 5 Venerupis

Water Change: Day 6

Initial Water Cu: 5.9 ppb

Water $\mathrm{Cu}$ at Water Change: $6.7 \mathrm{ppb}$

Low Copper Tank 3

5 Musculista, 5 Chione, 5 Venerupis

Water Change: Day 6

Initial Water Cu: $6.4 \mathrm{ppb}$

Water $\mathrm{Cu}$ at Water Change: $6.5 \mathrm{ppb}$

High Copper Tank 8

5 Musculista, 5 Chione, 5 Venerupis

Water Change: Day 6

\section{Initial Water Cu: 41.7 ppb}

Water Cu at Water Change: 29.9 ppb
High Copper Tank 9

5 Musculista, 5 Chione, 5 Venerupis

Water Change: Day 6

Initial Water Cu: 38.6 ppb

Water $\mathrm{Cu}$ at Water Change: $28.3 \mathrm{ppb}$

High Copper Tank 7

5 Musculista, 5 Chione, 5 Venerupis

Water Change: Day 6

Initial Water Cu: 41.9 ppb

Water Cu at Water Change: $31.3 \mathrm{ppb}$

Medium Copper Tank 4

5 Musculista, 5 Chione, 5 Venerupis

Water Change: Day 6

Initial Water Cu: Not measured

Water Cu at Water Change: $20.8 \mathrm{ppb}$
B

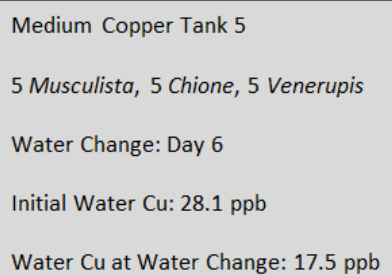

Low Copper Tank 2

5 Musculista, 5 Chione, 5 Venerupis

Water Change: Day 6

Initial Water Cu: $8.3 \mathrm{ppb}$

Water $\mathrm{Cu}$ at Water Change: $6.4 \mathrm{ppb}$

Low Copper Tank 3

5 Musculista, 5 Chione, 5 Venerupis

Water Change: Day 6

Initial Water $\mathrm{Cu}: 6.4 \mathrm{ppb}$

Water $\mathrm{Cu}$ at Water Change: $7.5 \mathrm{ppb}$

High Copper Tank 8

5 Musculista, 5 Chione, 5 Venerupis

Water Change: Day 6

Initial Water Cu: 47.8 ppb

Water $\mathrm{Cu}$ at Water Change: $51.1 \mathrm{ppb}$

\section{High Copper Tank 9 \\ 5 Musculista, 5 Chione, 5 Venerupis \\ Water Change: Day 6 \\ Initial Water Cu: $50.2 \mathrm{ppb}$ \\ Water $\mathrm{Cu}$ at Water Change: $38.4 \mathrm{ppb}$}

High Copper Tank 7

5 Musculista, 5 Chione, 5 Venerupis

Water Change: Day 6

Initial Water $\mathrm{Cu} 44.6 \mathrm{ppb}$

Water Cu at Water Change: $38.2 \mathrm{ppb}$

Medium Copper Tank 4

5 Musculista, 5 Chione, 5 Venerupis

Water Change: Day 6

Initial Water Cu: $30.5 \mathrm{ppb}$

Water $\mathrm{Cu}$ at Water Change: $21.8 \mu \mathrm{g} \mathrm{L}^{-1}$ 
Figure 1.4. Mean dissolved copper concentrations for initial water samples and difference between initial copper concentration and copper concentration at water change. Water change occurred on Day 6 of the experiment. Results from 18 day and 12 day experiments represented side by side for comparison. Error bars represent \pm one standard deviation. Differences were calculated by subtracting the copper concentration at time of water change from initial copper concentration. A positive value indicates that the copper concentration decreased from the initial concentration to the time of the water change, while a negative value indicates that the concentration at the time of the water change exceeded the initial copper concentration.

A. Mean initial copper concentrations for 18 and 12 day exposures for Low, Medium, and High copper tanks. $(\mathrm{N}=3)$

B. Mean difference between initial copper concentration and copper concentration at time of water change for 18 and 12 day exposures for Low, Medium, and High copper tanks. ( $N=3)$ 


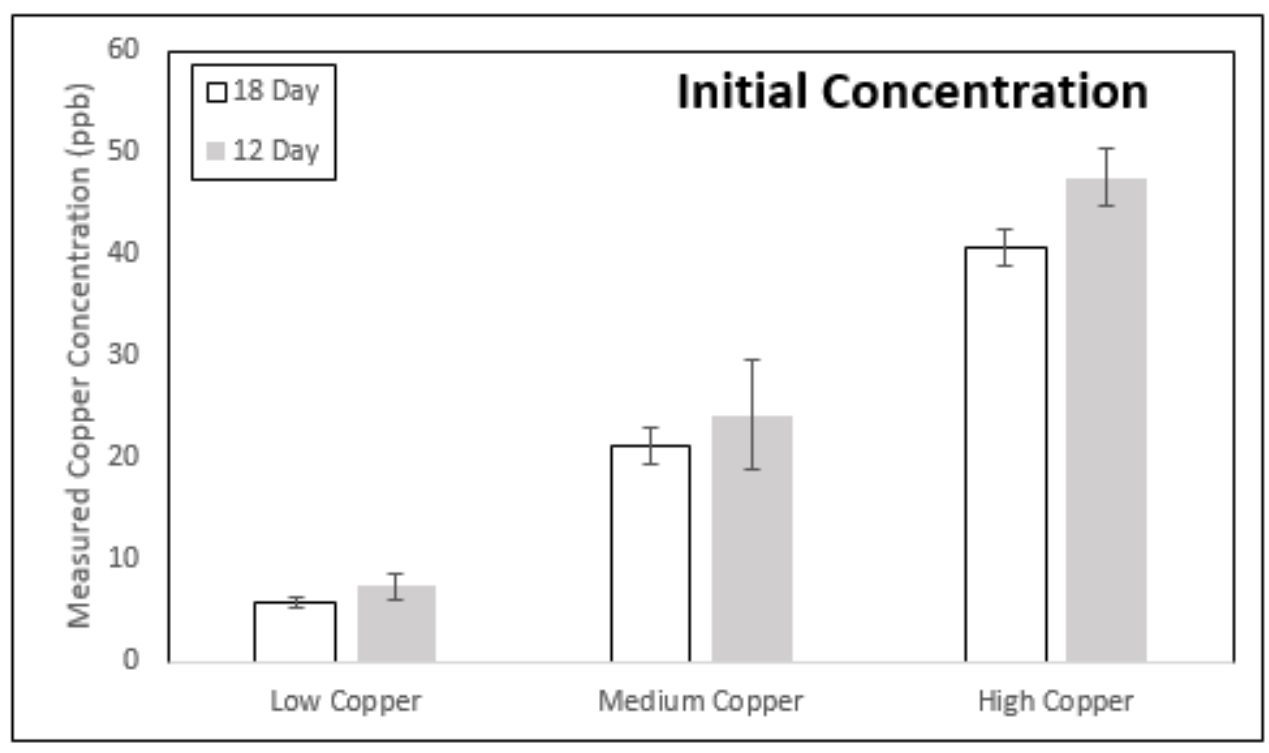

A

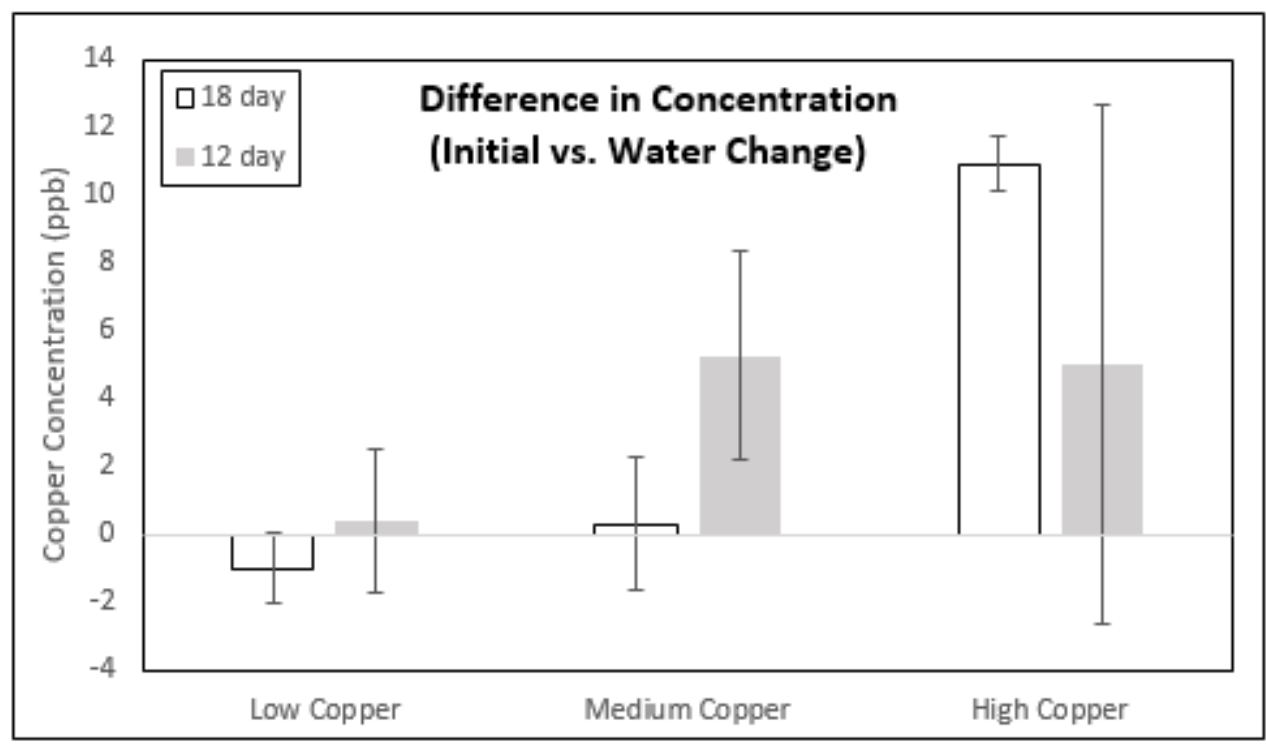

B 


\subsubsection{Tissue Copper}

The primary evidence of copper accumulation came in the examination of digested bivalve tissue. Significant differences were observed in whole body, gills and digestive tract. Most significant differences were observed after log transformation. Most notably, statistically significant increases were observed among copper concentrations across both experiments, and significant differences between species indicating Musculista accumulated more copper than Chione and Venerupis. However, most of the observed significant differences were not consistent across both experiments.

\subsubsection{Whole Body Tissue Copper}

There were increases in tissue copper concentrations as water copper concentration increased in both experiments, particularly for the 18 day exposure (Appendix B, Table 2.1, Figure 2.1). For each species, there was a clear increase in whole body copper concentration corresponding to an increase in copper exposure. There were some similarities, but also some noticeable differences between the species. Venerupis and Chione were very similar in terms of mass-specific copper concentration (Appendix B, Table 2.1, Figure 2.1). In comparison, Musculista had much higher average copper per dry mass than the other two species in both experiments (Appendix B, Table 2.1, Figure 2.1). 
Figure 2.1. Mean copper concentration per gram dry mass of specimens from the 18 day experiment. Error bars represent \pm one standard deviation, and $\mathrm{N}$ values varied based on survivorship (see Appendix B, Table 2.1). The p-values represent the statistically significant results of factorial ANOVA testing after a log transformation to normalize the data.

A.) Whole body copper per dry mass for Chione californiensis, Venerupis philippinarum, and Musculista senhousia.

B.) Gill copper per dry mass for Chione californiensis, Venerupis philippinarum, and Musculista senhousia.

C.) Digestive tract copper per dry mass for Chione californiensis, Venerupis philippinarum, and Musculista senhousia. 

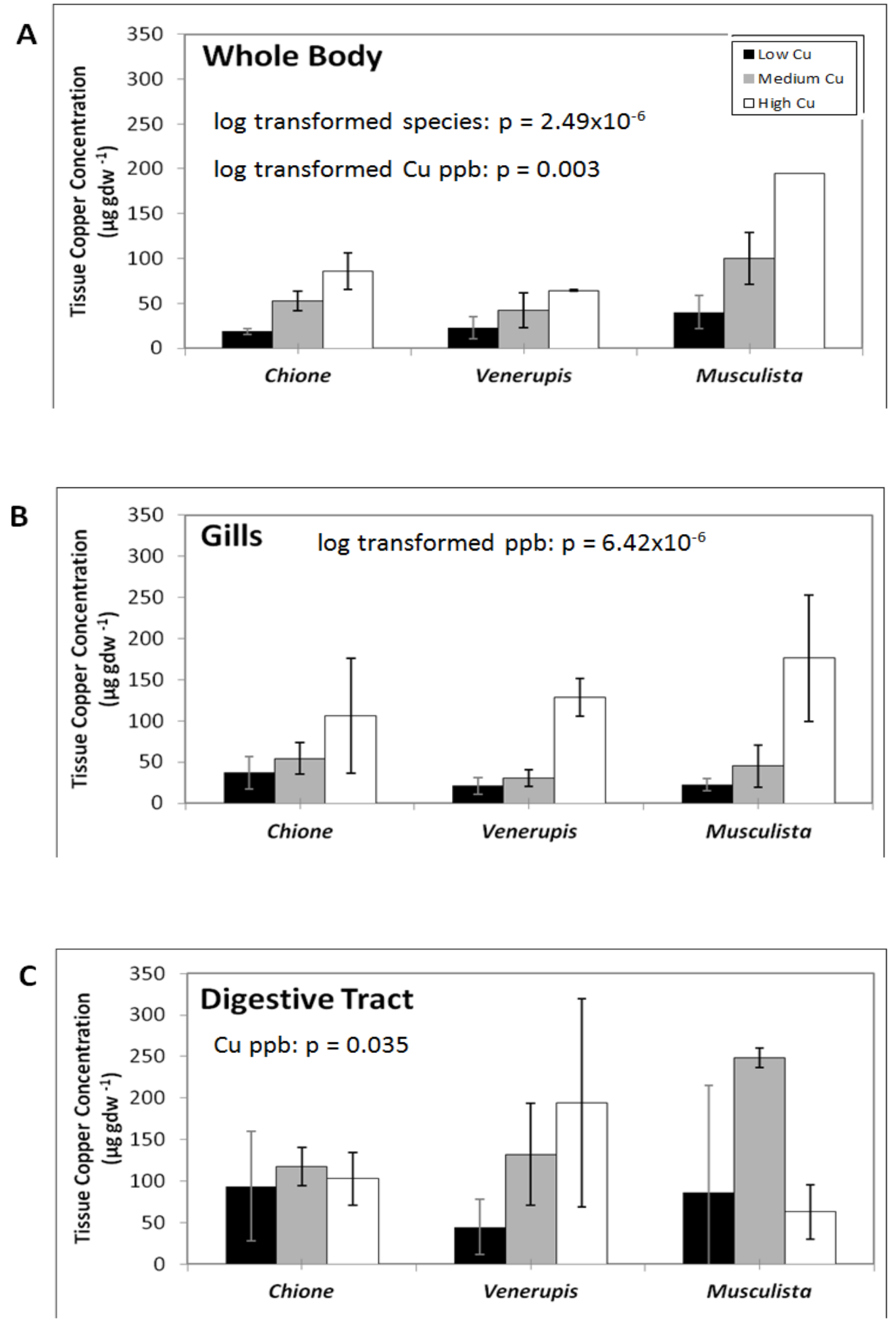
The 12 day exposure experiment showed a less clear pattern, as the whole body copper concentration for Chione in the Medium copper treatment group was greater than the concentration in the High copper treatment group (Appendix B, Table 2.1, Figure 2.2). Venerupis in the 12 day exposure, while showing a similar trend to the 18 day exposure, showed it in a less clear manner, as whole body tissue copper levels increased with increasing copper exposure. Both data sets were shown to be statistically significant (Appendix B, Table 2.1, Figure 2.2). The differences between Musculista and the other two species were also clear in the 12 day exposure, showing an increase in whole body copper as exposure increased (Appendix B, Table 2.1, Figure 2.2).

The distribution pattern of the whole body copper accumulation in relation to the rest of the data in this experiment was somewhat unusual. As with all of the other data sets, whole body copper accumulation was tested for normal distribution using the Shapiro-Wilk test. In the 18 day and 12 day exposures, the resulting $p$-values were 0.002 and $4.1 \times 10^{-4}$, respectively, indicating that neither data distribution was normal. Thus, statistical testing to determine if there were differences in whole body copper across species or copper concentrations required the use of nonparametric tests for both exposure periods. 
Figure 2.2. Mean copper concentration calculated for tissue per gram dry mass of specimens for the 12 day exposure experiment. Error bars represent \pm one standard deviation. $\mathrm{N}$ values were variable due to specimen mortality (see Appendix $\mathrm{B}$, Table 2.1). The $p$-values represent the statistically significant results of factorial ANOVA testing after a log transformation to normalize the data.

A.) Whole body copper per dry mass for Chione californiensis, Venerupis philippinarum, and Musculista senhousia.

B.) Gill copper per dry mass for Chione californiensis, Venerupis philippinarum, and Musculista senhousia.

C.) Digestive tract copper per dry mass for Chione californiensis, Venerupis philippinarum, and Musculista senhousia. 
A
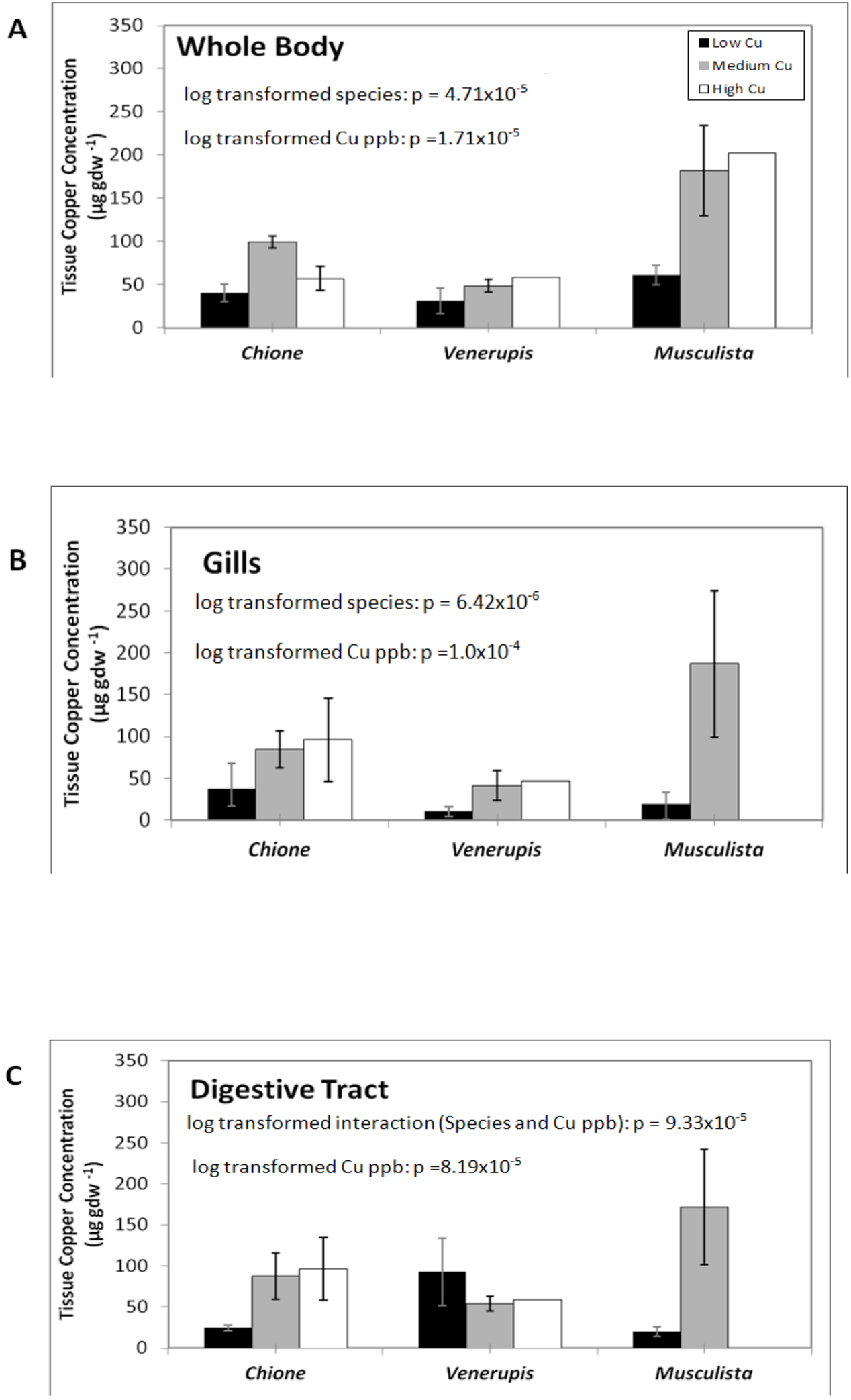
To normalize the whole body copper concentration data, a log transformation was used, and a factorial ANOVA was applied to the transformed data sets. Statistically significant differences were observed in the transformed data sets for treatment copper concentrations $(p=0.0026, F=9.10, D F=2)$ and species $\left(p=2.49 \times 10^{-6}, F=34.39, D F=\right.$ 2) for the 18 day exposure, and for treatment copper concentrations $\left(p=1.05 \times 10^{-5}, F\right.$ $=29.00, D F=2)$ and species $\left(p=2.60 \times 10^{-5}, F=24.62, D F=2\right)$ in the 12 day exposure. This indicates that both species and copper concentration showed statistically significant differences in whole body copper for both experiments. In neither the 18 day nor the 12 day experiment was the interaction term between measured copper exposure and species statistically significant $(p=0.755$ and 0.083 , respectively, $D F=3$ )

Post hoc testing using Tukey's HSD revealed that among the individual category for species in the 18 day experiment, there was a statistically significant difference in whole body tissue copper accumulation between Chione and Musculista $(p=0.021)$, and between Venerupis and Musculista $(p=0.002)$, but not between Chione and Venerupis $(p=0.423)$. For the copper concentration data, Tukey's HSD showed that there were statistically significant differences between treatment copper concentrations: Low vs. Medium $\left(p=1.4 \times 10^{-5}\right)$, Low vs. High ppb $\left(p=3.0 \times 10^{-7}\right)$, and Medium vs. High $(p=0.029)$.

Similar results were observed for the 12 day experiment. For the species factor, significant differences were observed between Chione and Musculista $\left(p=7.2 \times 10^{-4}\right)$ and Venerupis and Musculista $\left(p=7.5 \times 10^{-6}\right)$, but unlike the 18 day experiment, a statistically significant difference was observed between Chione and Venerupis $(p=0.019)$. For the copper concentration data, there were statistically significant differences between the 
Low and Medium $\left(p=2.0 \times 10^{-5}\right)$ and the Low and High copper treatment groups $(p=$ $0.002)$, but not the Medium and High copper groups $(p=0.274)$.

\subsubsection{Gill Tissue Copper}

A consistent increase in gill tissue copper concentration was observed in both experiments (Figure 2.1, Figure 2.2). Comparing across species, gill copper showed many of the same trends as whole body copper accumulation, with Musculista exhibiting the highest tissue copper concentrations, and Chione and Venerupis being lower and fairly similar. This was true for both the 18 and 12 day experiments: Chione had gill copper concentrations that were higher than Venerupis at the end of the 12 day experiment across all three exposures, though the difference was not statistically significant (Figure 2.2). Gill copper concentrations were not normally distributed for the 18 and 12 day experiments and thus had to be log transformed, after which they demonstrated a normal distribution (Shapiro-Wilk test, $p=0.122$ and 0.421 , respectively). After transformation of the gill copper concentration data from both experiments, a factorial ANOVA was deemed appropriate.

A factorial ANOVA of the log-transformed data showed that there was a significant difference between gill copper concentrations at different copper exposure levels in the 18 day experiment $\left(p=6.42 \times 10^{-6}, F=27.66, D F=2\right)$ and the 12 day experiment $(p=0.015, F=5.78, D F=2)$. In the 18 day experiment, there was no significant difference in gill copper concentrations among species $(p=0.168, F=2.00, D F$

=2). The lack of statistical significance could be the result of a low $N$ for Musculista at higher copper concentrations (Figure 2.2). For the 12 day experiment, the gill copper data did show a significant difference between species $\left(p=1.0 \times 10^{-4}, F=18.96, D F=2\right)$. 
Neither experiment showed a significant interaction $(p=0.265, D F=3$ and 0.328 , $\mathrm{DF}=3)$.

Post hoc testing for the 18 day experiment was done to further examine differences between groups exposed to different copper concentrations. Tukey's HSD revealed statistically significant differences in gill copper accumulation between the Low and High $\left(p=4.9 \times 10^{-6}\right)$ and the Medium and High copper treatment groups $(p=0.0003)$, but not between the Low and Medium copper groups $(p=0.067)$.

The gill copper accumulation for the 12 day experiment in relation to copper concentration was similar to that of the 18 day experiment, as both showed significant differences indicating a positive correlation between copper accumulation and copper exposure, although between different copper concentrations. Of the copper accumulation for gills and digestive tract, the 12 day exposure for the gills was the only example that showed a significant difference between the species. There were significant differences between Venerupis and Musculista, and Chione and Venerupis, with Venerupis consistently exhibiting a lower accumulation than either of the other two species. However, the lack of statistical significance between species for the gills in the 18 day experiment makes it difficult to draw firm conclusions about the relationship between copper exposure and gill accumulation. The 12 day experiment showed significant differences between Venerupis and Chione $(p=0.016)$ and between Venerupis and Musculista $(p=0.054)$ but not Musculista and Chione $(p=0.933)$. There were significant differences across species between the Low and Medium $(p=0.00012)$ and Low and High copper treatment groups $(p=0.004)$ but not between the Medium and High copper groups $(p=0.823)$. 


\subsubsection{Digestive Tract Tissue Copper}

Digestive tract tissue did not exhibit the same trends as whole body and gill tissue copper, and there was not a positive correlation between copper exposure and tissue copper. There were some exceptions to this pattern, however. Venerupis for the 18 day exposure showed a positive correlation, as did Chione in the 12 day experiment (Figure 2.1, Figure 2.2). There were no data for the High copper treatment for Musculista, but the Low and Medium groups showed an increase in copper accumulation as exposure increased. Digestive tract copper concentrations were normally distributed for the 18 day experiment but not for the 12 day experiment, and these data were log-transformed for analysis (Shapiro-Wilk test, $p=0.063$ and $p=0.574$ before and after log transformation, respectively). Thus, a factorial ANOVA could be used to test the independent factors of species and copper exposure against the response variable of tissue copper concentration.

After running a factorial ANOVA on the untransformed 18 day digestive tract copper data, there was a statistically significant difference between copper concentration treatments $(p=0.035, F=4.176, D F=2)$, but not between species $(p=$ $0.531, F=0.658, \mathrm{DF}=2$ ). Post hoc testing using a Tukey's HSD showed that there was significant difference between only the Low and Medium copper groups $(p=0.027)$. There were no significant differences in Low vs. High copper and Medium vs. High copper comparisons $(p=0.400$ and $p=0.376)$. There were no statistically significant differences between species for the 18 day experiment $(p=0.531, F=0.658, D F=2)$, nor was the interaction between species and copper concentration significant $(p=$ $0.072, F=2.652)$ 
The 12 day digestive tract copper data, after being log transformed, were normally distributed, and a factorial ANOVA was performed. Though there were no obvious trends from the graphs produced, the ANOVA did determine statistically significant differences between both the copper concentrations $\left(p=8.19 \times 10^{-5}, F=\right.$ $19.85, D F=2)$ and the interaction between species and copper concentration $(p=9.325$ $\left.x 10^{-5}, F=15.70, D F=2\right)$. Further examination of these differences using a Tukey's HSD post hoc test revealed that the significant differences were between the Low and Medium $\left(p=1.1 \times 10^{-4}\right)$ and Low and High copper treatments $(p=0.002)$ but not between the Medium and High copper treatments $(p=0.898)$. There was no significant difference between species $(p=0.63, F=0.477, D F=2)$.

After running the factorial ANOVA on the data, there was no statistical support for the trends seen in the graphs, as $p$-values were high across experiments for both independent factors. For species, statistical significance was not observed in either the 18 day $(p=0.89, F=0.122, D F=2)$ or 12 day $(p=0.858, F=0.161, D F=2)$ experiment. For copper exposure, the 18 day $(p=0.372, F=1.280, D F=2)$ and 12 day $(p=0.533, F=$ $0.783, \mathrm{DF}=2$ ) experiments also did not show statistically significant results.

In the 18 day experiment, the log transformed total gill copper showed significant differences between copper exposure groups. The 18 day digestive tract copper data did not require transformation to achieve a normal distribution and showed statistically significant differences between copper exposure groups as well. The $p$ values for gill copper were much lower than those for the digestive tract, suggesting stronger differences among treatments for gill copper. 
The 12 day digestive tract data showed similar patterns of significant difference between copper concentrations, but unlike all the other copper accumulation data, the 12 day digestive tract showed a statistically significant interaction between species and copper concentration.

\subsubsection{Survivorship}

Survivorship was broken down into two main comparisons: survivorship rates at various copper exposure levels compared within species, and survivorship at various copper levels compared between species. Initial examinations of the data revealed that the mortality rates differed between the two experiments. Despite having a longer exposure period, the 18 day exposure showed higher survivorship for all three species in the High copper tanks than the 12 day experiment (Figure 3.1A, Figure 3.1B). Musculista in the Medium copper tanks for the 18 day experiment also exhibited much higher survivorship than the corresponding tanks for the 12 day experiment. In most instances, copper concentration was negatively correlated with survival rates (Figure 3.1A and 3.1B). Survivorship was lowest in the High copper treatments for all species in both experiments. In the 12 day experiment, survival rate for Chione was slightly higher in the Medium vs. the Low copper tanks.

Across all species in both experiments, there was always a greater or comparable decrease in survivorship between the Medium and High copper treatments than between the Low and Medium copper treatments. The mean survivorship for the 18 day experiments fell by $6.6 \%, 0 \%$, and $13.3 \%$ between Low and Medium copper treatments for Chione, Musculista, and Venerupis, respectively (Appendix B, Table 3.1). 
Between the Medium and High copper treatments, survivorship numbers fell much more sharply, with decreases of $13.3 \%, 66.7 \%$, and $60.0 \%$ for Chione, Musculista, and Venerupis, respectively. For the 12 day experiment, a similar trend was observed. Survivorship percentages changed by $-0.67 \%, 40.0 \%$, and $20.0 \%$ between the Low and Medium copper treatments for Chione, Musculista, and Venerupis (Appendix B, Table 3.1). The Medium and High copper treatments showed survivorship differences of 53.3\%, 53.3\%, and 60.0\% (Appendix B, Table 3.1). The data show that survivorship did not decrease in a linear fashion as copper concentration increased, which suggests that mortality in relation to copper may be nonlinear.

A Shapiro-Wilk test for normality gave a $p$-value of $6.2 \times 10^{-4}$ for the survivorship data in the 18 day experiment and $3.2 \times 10^{-4}$ for the 12 day experiment. A Kruskal-Wallis test was used to analyze the dependent variable, the arcsine transformed survivorship rate, against two independent variables: species and copper exposure levels. 
Figure 3.1. Survivorship percentages over experiment duration. Survivorship percentage combined all members of individual species at a single exposure level across the three tanks dosed at that level. For $\mathrm{N}$ values, refer to Appendix B, Table 3.1. Error bars represent \pm one standard deviation. The $p$-values represent the statistically significant results of Kruskal-Wallis testing, as the data distribution was not normal.

A. 18 day exposure survivorship comparison between Chione californiensis, Venerupis philippinarum, and Musculista senhousia in the Low, Medium and High copper treatment groups.

B. 12 day exposure survivorship comparison between Chione californiensis, Venerupis philippinarum, and Musculista senhousia in the Low, Medium and High copper treatment groups.

C. Survivorship percentages for both exposure periods through 12 days with Chione californiensis, Venerupis philippinarum, and Musculista senhousia in the Low, Medium and High copper treatment groups. Survivorship data from the 18 day exposure were calculated after 12 days to facilitate combination with 12 day exposure data, as the data for corresponding days were combined to take the mean of both exposure periods. 
A

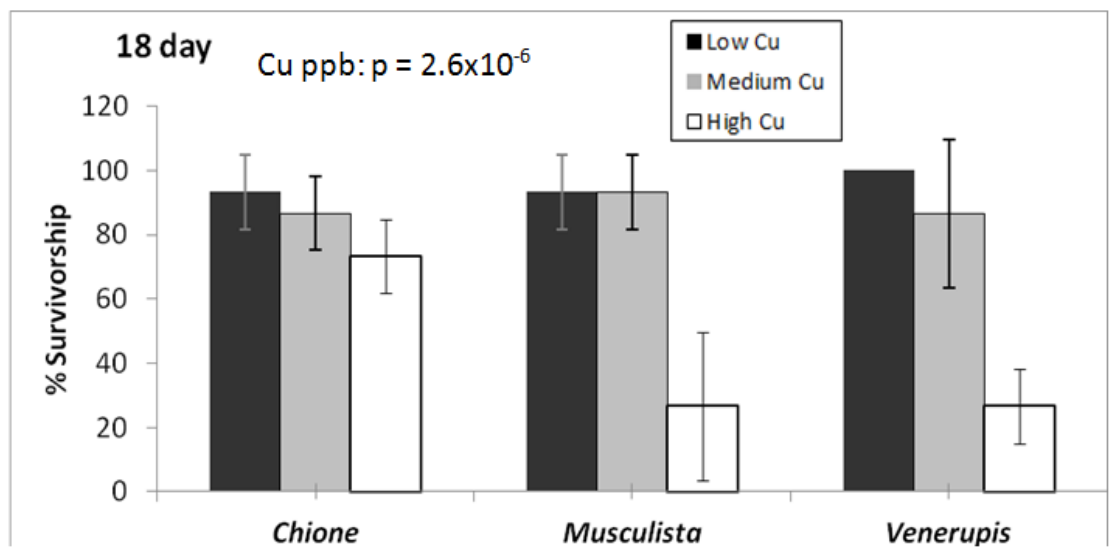

B

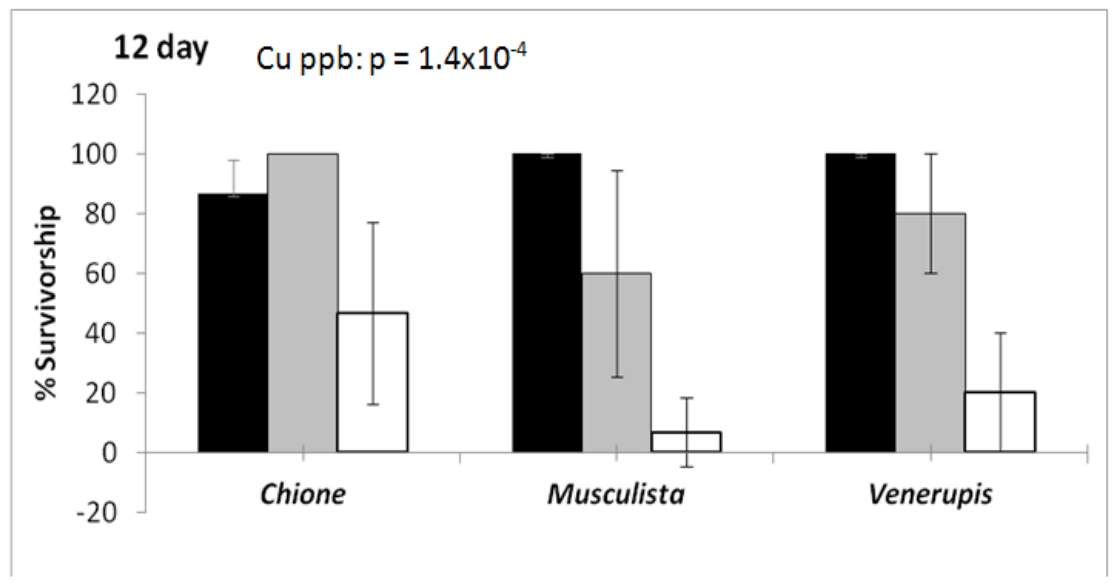

C

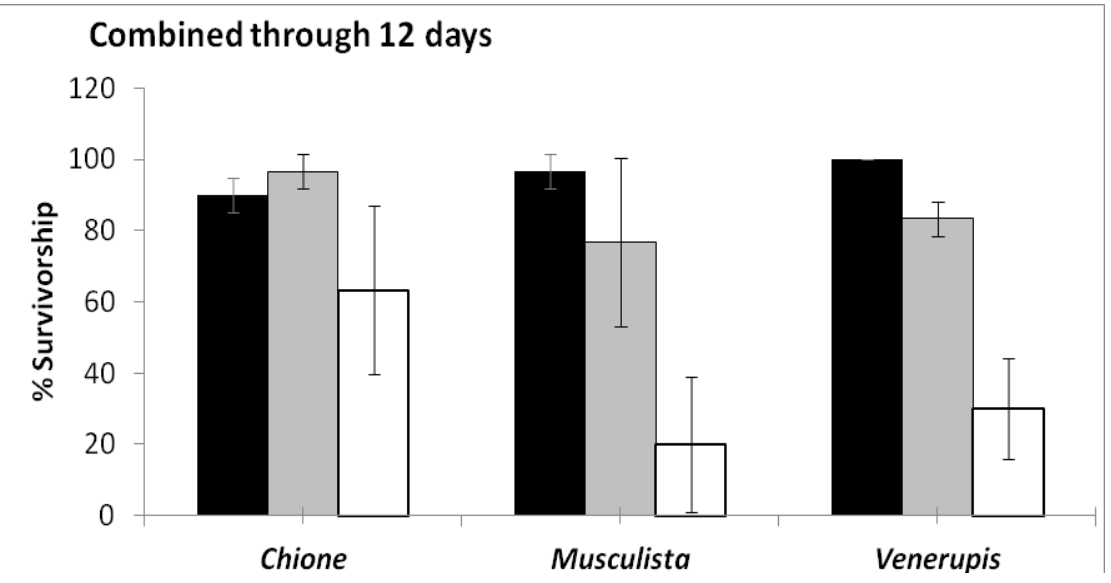


A statistically significant relationship was identified between copper exposure and survivorship in both the 18 day $\left(p=2.6 \times 10^{-6}, D F=2\right)$ and 12 day experiments $(p=$ $\left.1.4 \times 10^{-4}, \mathrm{DF}=2\right)$, and a Dunn test with Bonferroni correction showed significant differences between the High copper group and both the Low $(p=0.002$ and $p=1.0 \times 10$ ${ }^{4}$ ) and Medium copper groups ( $p=0.003$ and $p=0.009$ ) for both the 18 day and 12 day experiments, but there was no significant difference between the Low and Medium copper groups in either experiment $(p=0.691$ and $p=0.246)$. Survivorship differences also were observed between species. In both the 18 day and 12 day experiments, the survival rate of Chione californiensis was higher than that of the other two species in the High copper treatment. Chione had a survival percentage of $73.3 \%$ for the 18 day exposure and $46.7 \%$ for the 12 day exposure (Appendix B, Table 3.1, Figure 3.1A, and Figure 3.1B). Each of these survivorship percentages was more than $20 \%$ higher than the survivorship rates for either of the other two species,. However, across both experiments the species did not show significant differences between one another in survivorship ( $p=0.790$ and 0.597 , respectively). Thus, despite the apparent trends, there was no statistically significant difference between Chione and the other species.

When combining the 18 and 12 day survival rates, however, an ANOVA showed that there was a strong statistical correlation between species and survivorship in the High copper treatment, with a p-value of 0.009 based on the 18 day data, and a p-value of 0.024 based on data from the 12 day mark of the 18 day experiment. A Tukey's post hoc test showed that there was a statistically significant difference between Chione and Venerupis in the data with a p-value of 0.052 , but when the 18 day experiment was 
normalized, the $p$-value rose to 0.093 , showing no statistical difference between the two. However, there were statistical differences between Chione and Musculista in both the unaltered data set with a p-value of 0.0086 , and a p-value of 0.022 in the 12 day subsample of the 18 day data. This is perhaps the clearest representation of species difference in reaction to copper exposure in this study.

\subsubsection{Survivorship over time}

Survivorship was examined in several ways, one of which was plotting mortality of the bivalve species over time. As previously mentioned, mortality trends were different between the two exposure periods. For the Low and Medium copper treatments, there was some consistency across species. In tanks that exhibited elevated mortality, bivalve deaths generally began around day 8 (Figures 3.2, 3.3, 3.4, 3.5), with the exception of Musculista in the 12 day exposure, where there was a sharp increase in mortality by day 3 (Figures 3.4, 3.5).

For the High copper treatment, bivalve deaths typically began between days 3 and 5 (Figures 3.2, 3.4). Unlike the lower concentrations, which usually were associated with brief (one to two day) periods of elevated mortality rates and longer intervals with no mortality, the highest concentration group typically showed longer, more sustained periods of elevated mortality. For instance, the Venerupis High copper treatment (15 individuals) exhibited at least one mortality per day over a seven day period in the 18 day exposure experiment, and the Venerupis High copper treatment in the 12 day experiment exhibited mortality for five of the final six days of the 12 day exposure (Figures 3.2, 3.4). 
Figure 3.2. Survivorship percentage over time for 18 day exposure of all species at various estimated copper exposure levels. Data combined for total survivorship percentage across the three exposure tanks within each concentration. The vertical line at day 6 represents the day when $100 \%$ of the water was changed.

A. Daily survivorship for Chione californiensis, Venerupis philippinarum, and Musculista senhousia in the Low copper treatment group.

B. Daily survivorship for Chione californiensis, Venerupis philippinarum, and Musculista senhousia in the Medium copper treatment group.

C. Daily survivorship for Chione californiensis, Venerupis philippinarum, and Musculista senhousia in the High copper treatment group. 


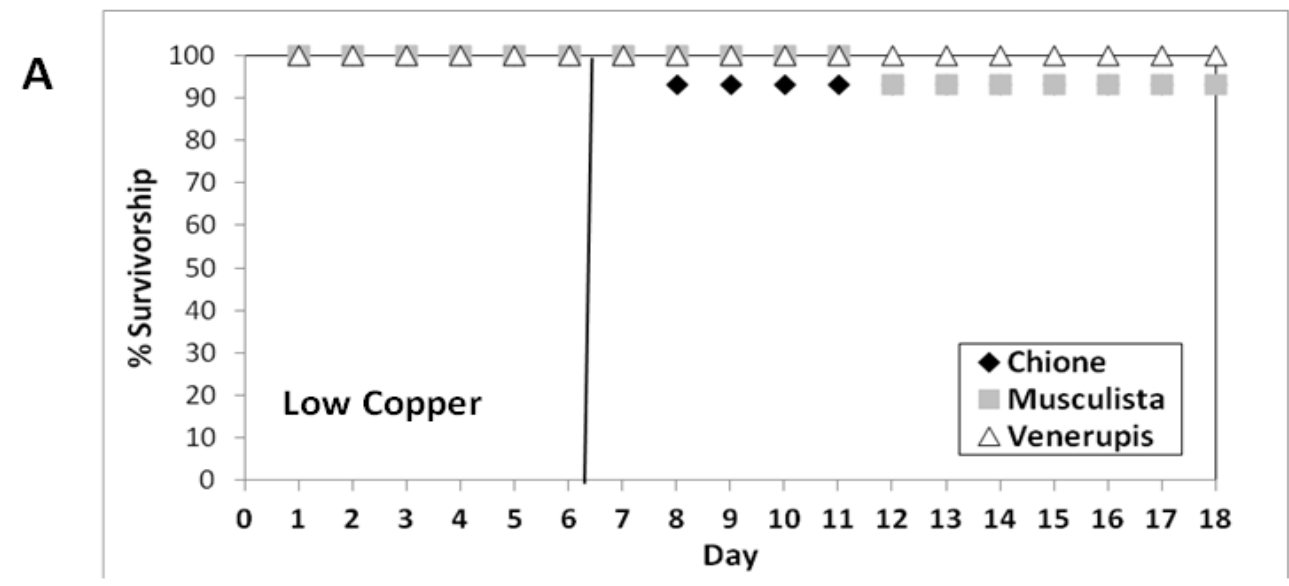

B

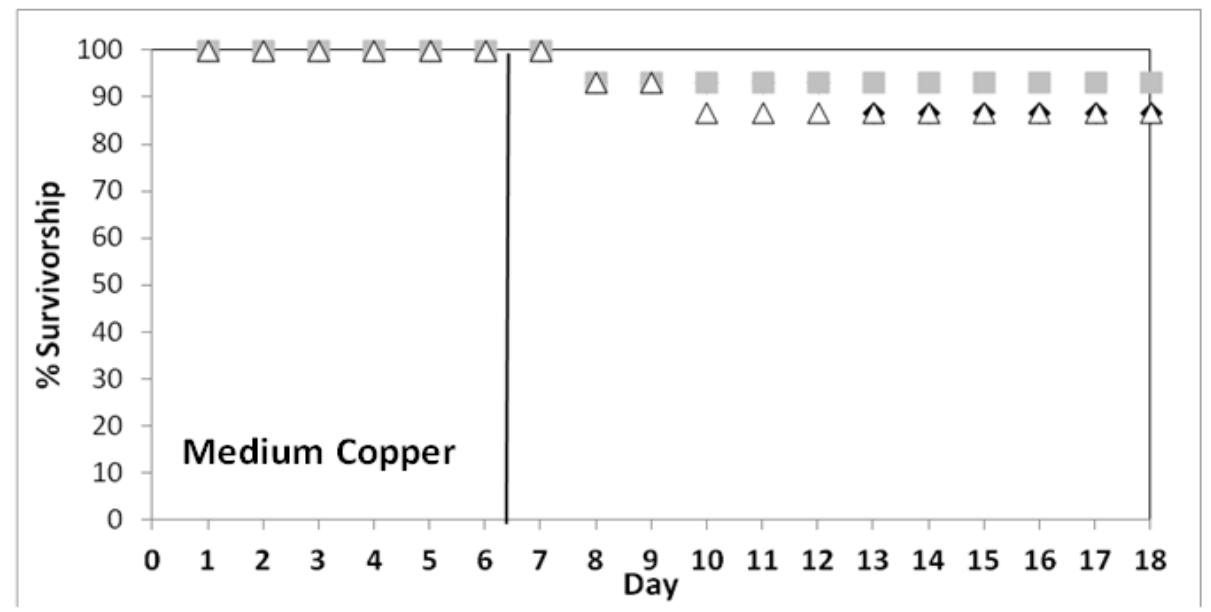

C

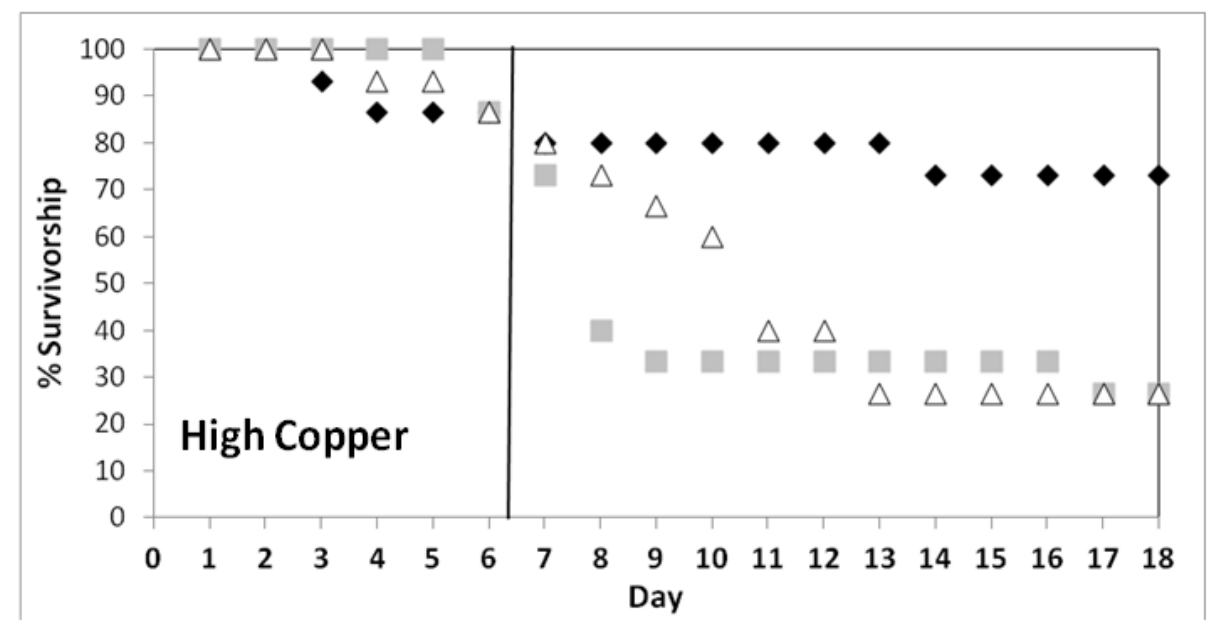


Figure 3.3. Survivorship percentage over time for 18 day exposure of all species at various estimated copper exposure levels. Data combined for total survivorship percentage across the three exposure tanks within each concentration. The vertical line at day 6 represents the day when $100 \%$ of the water was changed.

A. Daily survivorship for Chione californiensis in the Low, Medium and High copper treatment groups.

B. Daily survivorship for Venerupis philippinarum in the Low, Medium and High copper treatment groups.

C. Daily survivorship for Musculista senhousia in the Low, Medium and High copper treatment groups. 


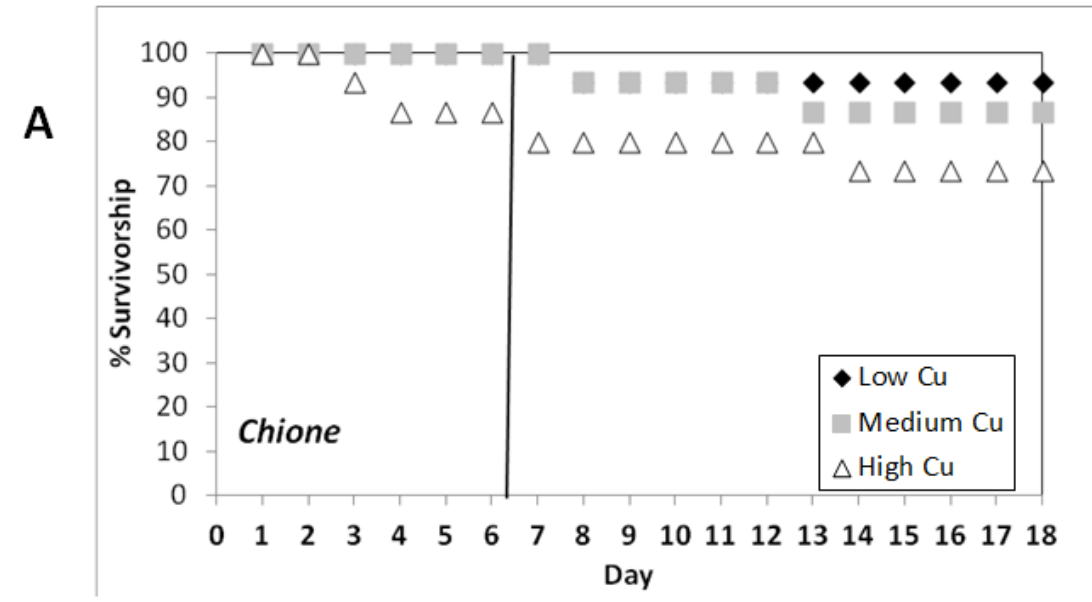

B
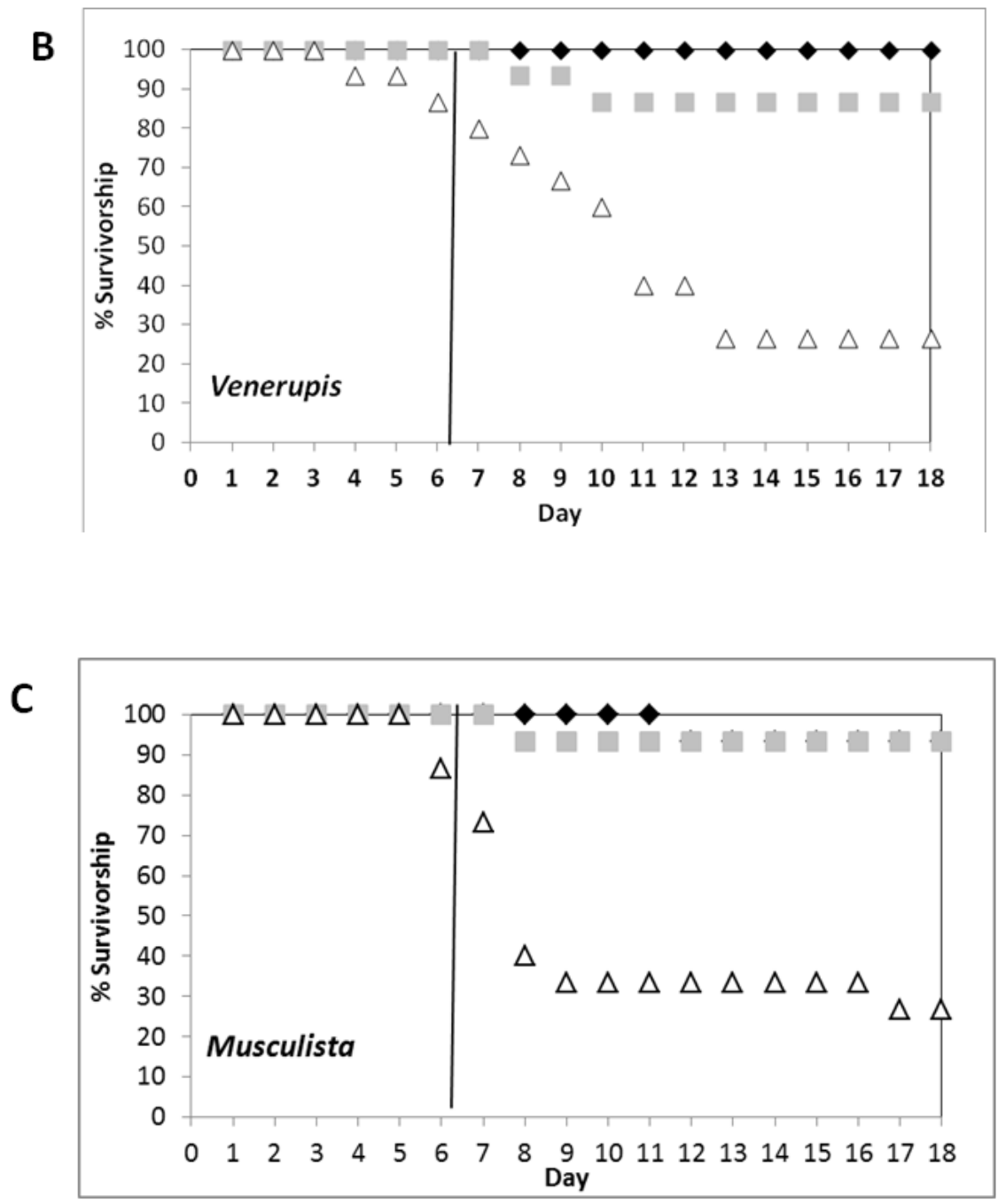
Figure 3.4. Survivorship percentage over time for 12 day exposure of all species at various estimated copper exposure levels. Data combined for total survivorship percentage across the three exposure tanks within each concentration. The vertical line at day 6 represents the day when $100 \%$ of the water was changed.

A. Daily survivorship for Chione californiensis, Venerupis philippinarum, and Musculista senhousia in the Low copper treatment group.

B. Daily survivorship for Chione californiensis, Venerupis philippinarum, and Musculista senhousia in the Medium copper treatment group.

C. Daily survivorship for Chione californiensis, Venerupis philippinarum, and Musculista senhousia in the High copper treatment group. 

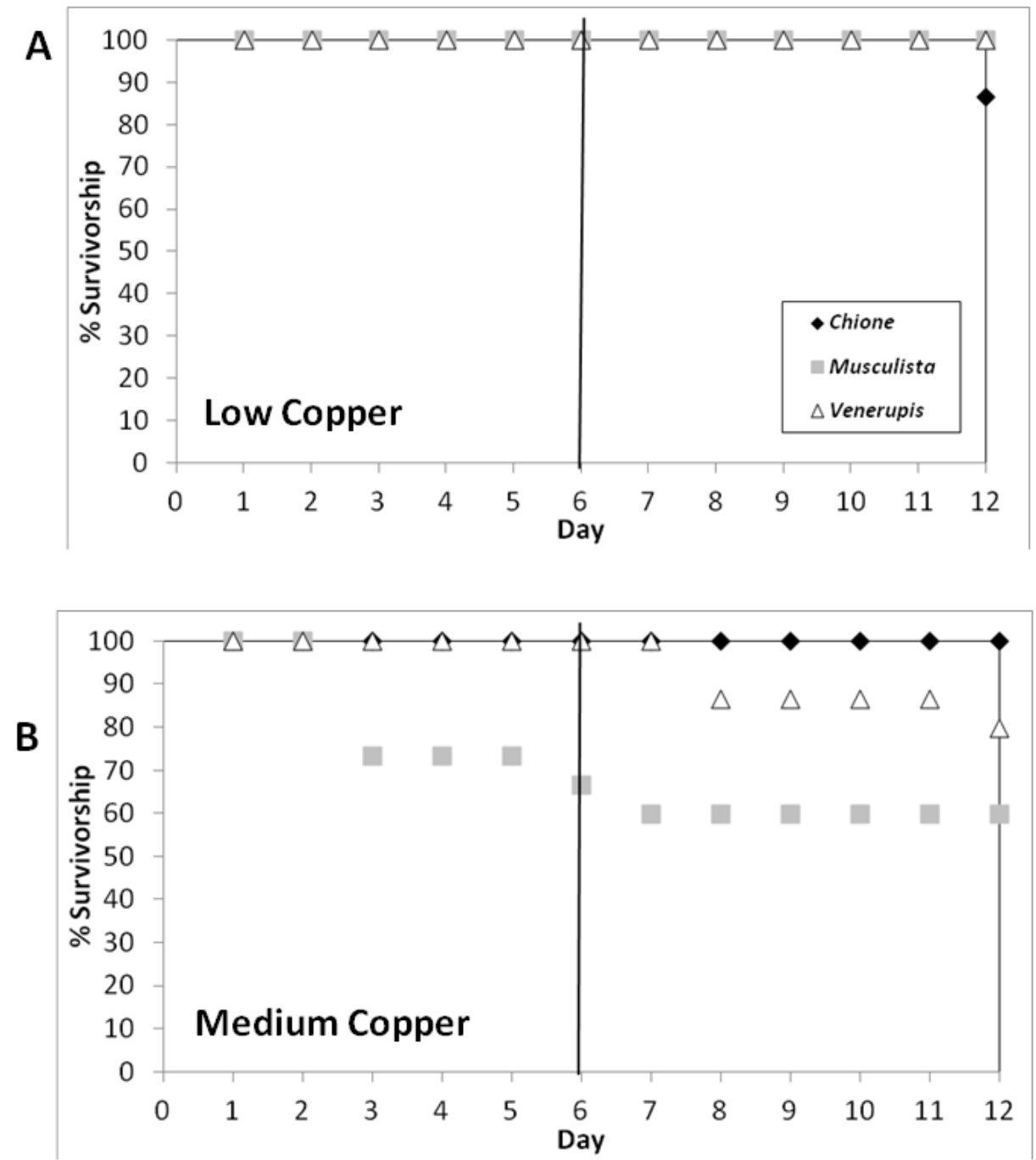

C

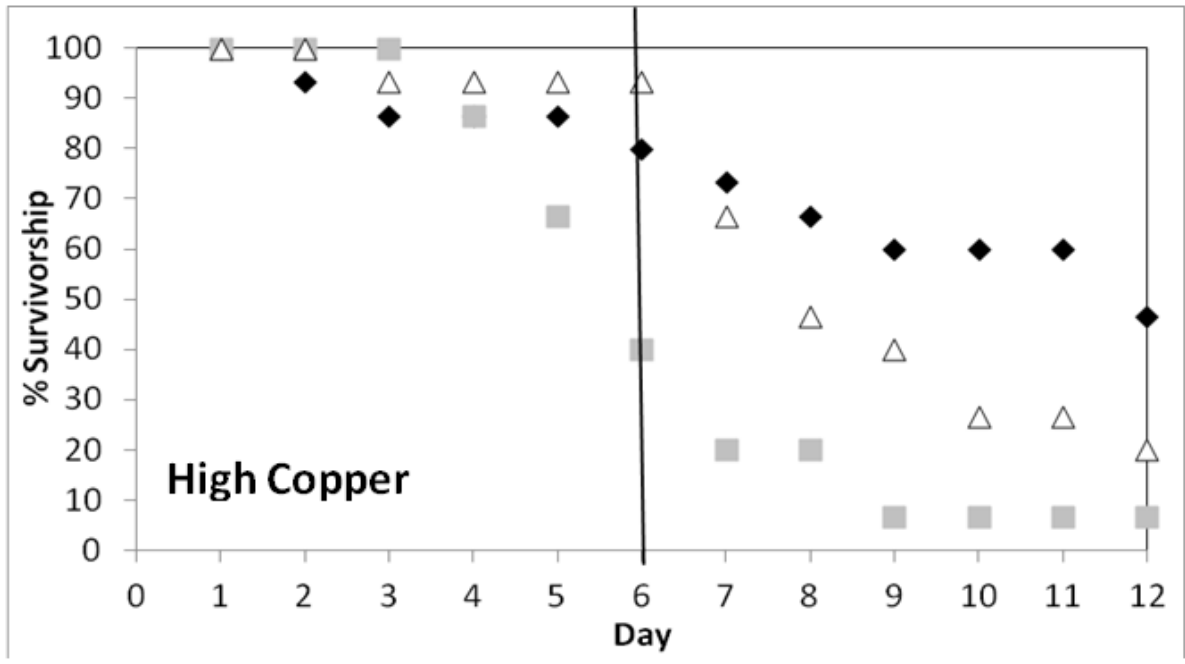


Figure 3.5. Survivorship percentage over time for 18 day exposure of all species at various estimated copper exposure levels. Data combined for total survivorship percentage across the three exposure tanks within each concentration. The vertical line at day 6 represents the day when $100 \%$ of the water was changed.

A. Daily survivorship for Chione californiensis in the Low, Medium and High copper treatment groups.

B. Daily survivorship for Venerupis philippinarum in the Low, Medium and High copper treatment groups.

C. Daily survivorship for Musculista senhousia in the Low, Medium and High copper treatment groups. 


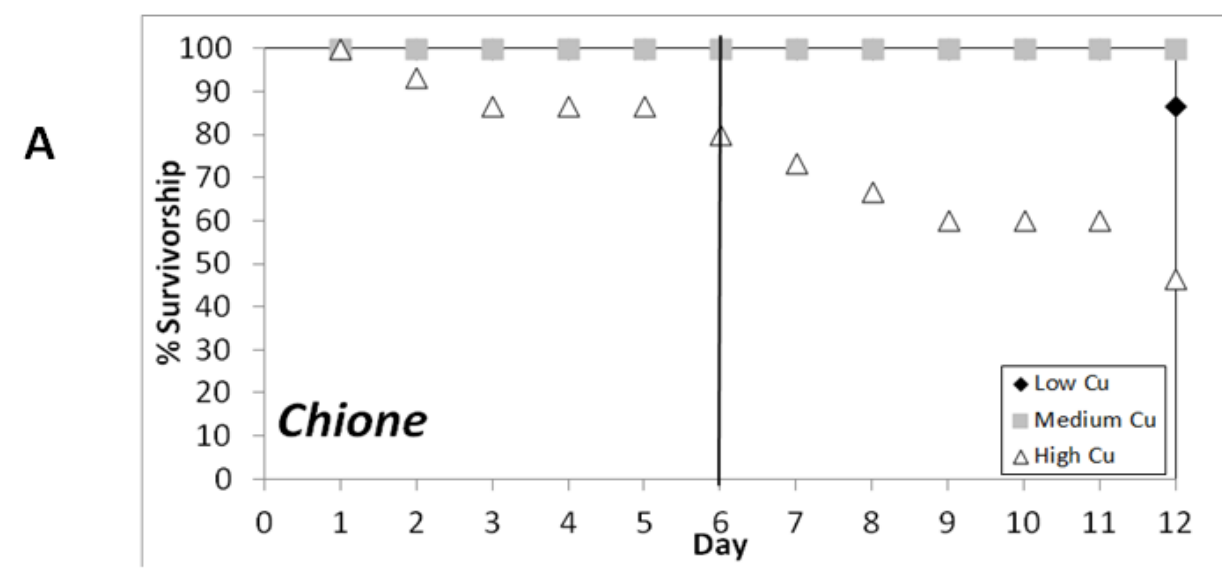

B

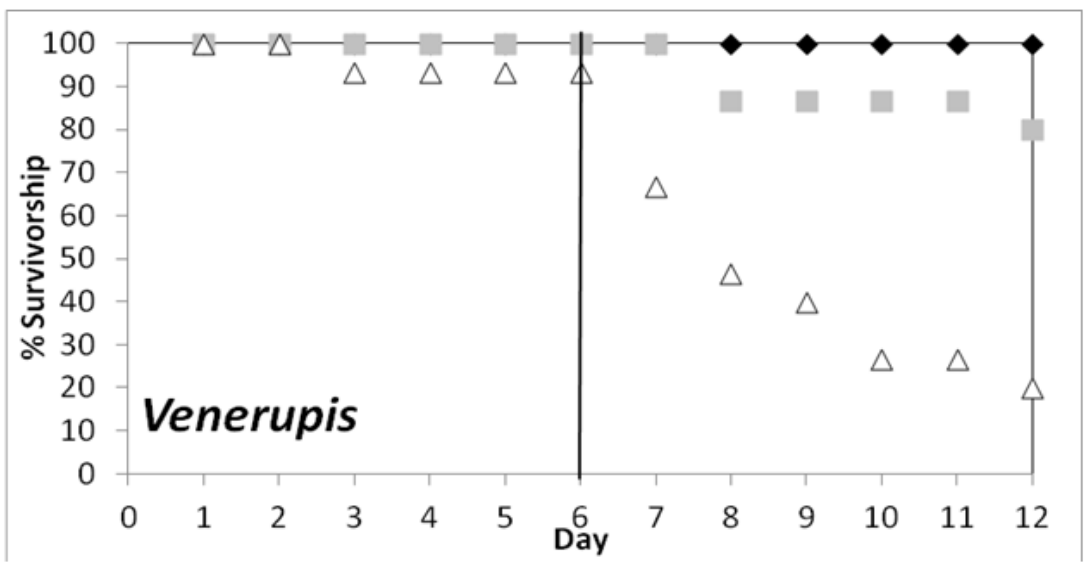

C

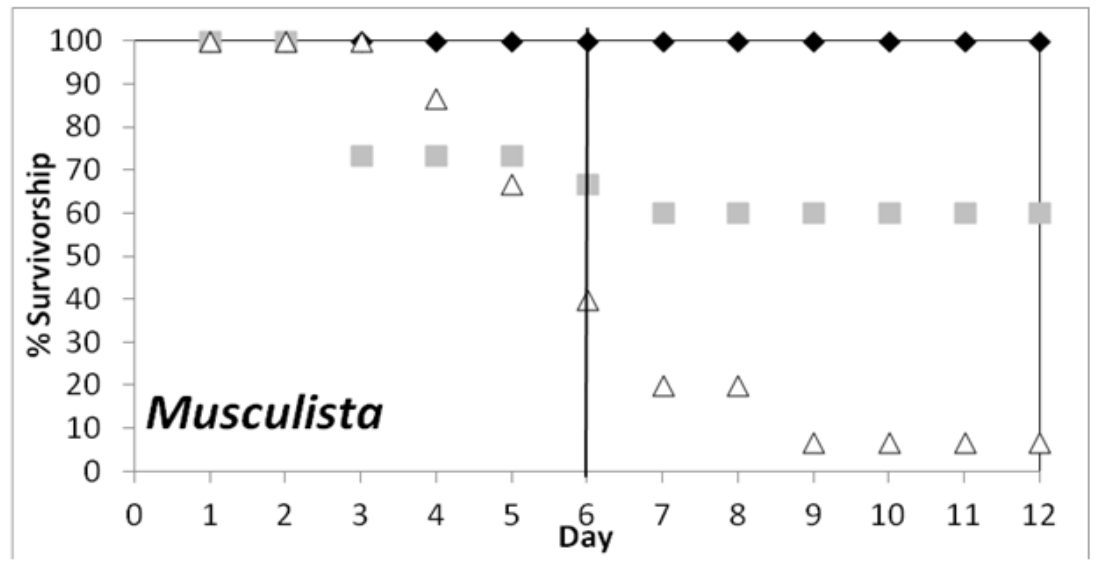




\subsubsection{Survivorship by tank}

To evaluate whether unusual mortality within specific tanks may have affected the results of the pooled survivorship estimates for these experiments, survivorship was examined on a tank by tank basis. In general, there appeared to be consistent trends in mortality across tanks (Figures 3.8, 3.9). The Low and Medium copper treatment tanks for both experiments showed mortalities of individuals as isolated events: a bivalve would die and be removed, followed by several days before the next mortality. By contrast, the High copper treatment tanks showed a consistent trend of multiple bivalves dying on the same day or within a day or two of one another.

One tank exhibited unusual survivorship data. Tank 5 (Medium copper) of the 12 day experiment showed an $80 \%$ die-off of Musculista by day 2 (Figure 3.9). This severe die-off did not occur in any of the other Medium copper tanks in the same experiment, nor those in the 18 day exposure. Only one other mortality occurred in the tank for the duration of the experiment.

Another observation was an increase in mortality following a water change in the Medium and High copper treatment tanks. This was observed in Musculista and Venerupis primarily, although there were also instances of Chione mortality immediately after water changes in the High copper tanks (Figures 3.8, 3.9). Copper concentrations immediately before the water change in most tanks were lower than initial copper concentrations on Day 0 (Appendix B, Table 1.1). 
Figure 3.6 Mean survivorship percentage of Chione californiensis, Venerupis philippinarum, and Musculista senhousia compared to mean copper per dry mass of whole body, gills and digestive tract for individuals in the 18 day exposure experiment. Individual bivalves were exposed to Low, Medium and High copper treatments and underwent a full water change on day 6 .

A. Survivorship percentage vs. whole body copper per dry mass.

B. Survivorship percentage vs. gill copper per dry mass.

C. Survivorship percentage vs. digestive tract copper per dry mass. 

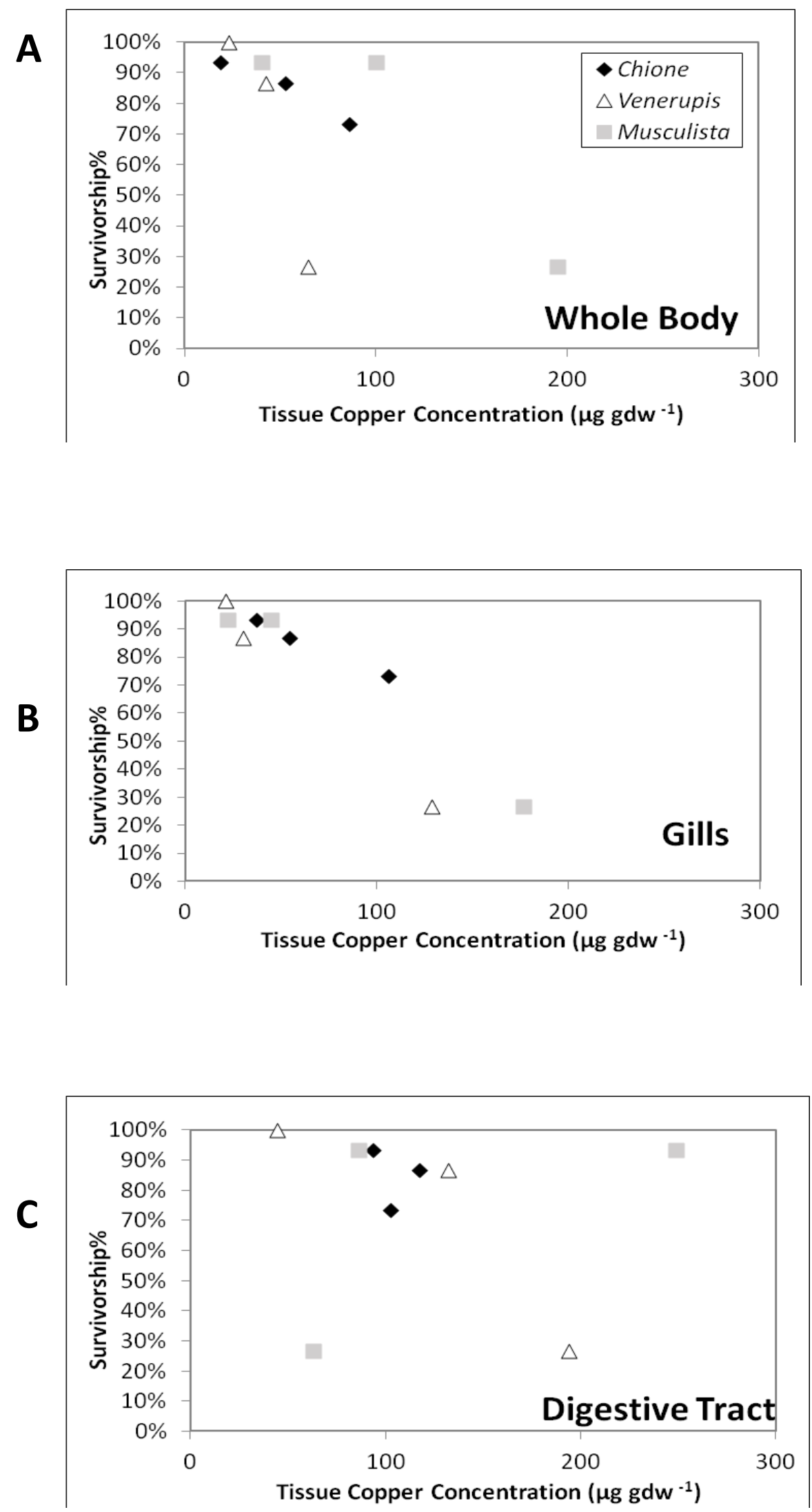
Figure 3.7 Mean survivorship percentage of Chione californiensis, Venerupis philippinarum, and Musculista senhousia compared to mean copper per dry mass of whole body, gills and digestive tract for individuals in the 12 day exposure experiment. Initial n value was 5 per tank, and survivorship percentage was calculated for each tank and then averaged to produce the mean survivorship percentage. Individual bivalves were exposed to Low, Medium and High copper treatments and underwent a full water change on day 6.

A. Survivorship percentage vs. whole body copper per dry mass.

B. Survivorship percentage vs. gill copper per dry mass.

C. Survivorship percentage vs. digestive tract copper per dry mass. 

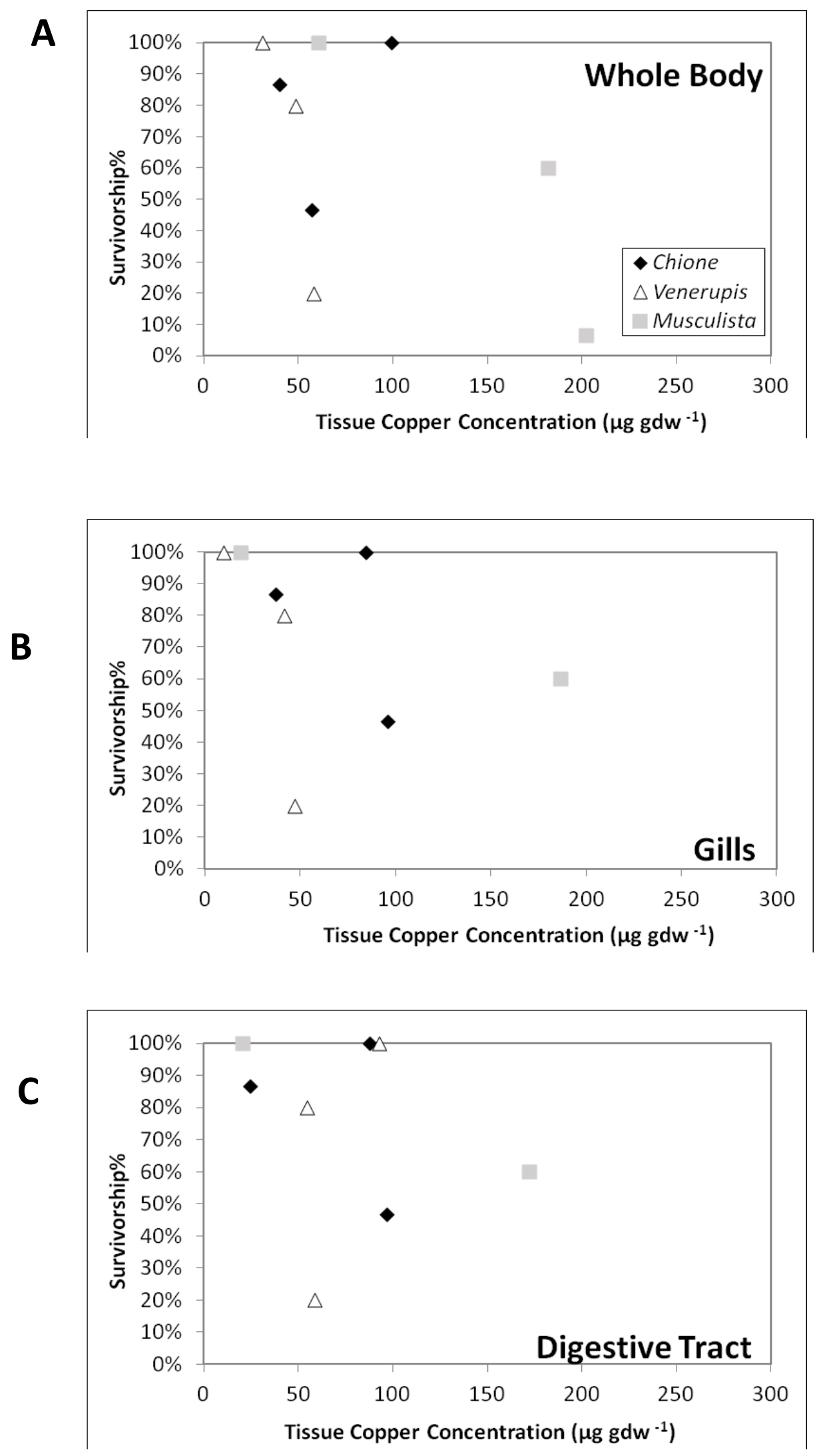
Figure 3.8 Survivorship percentages over time for each tank in the 18 day experiment, including all species at various estimated copper exposure levels. Tanks 1, 2, and 3 represent the Low copper treatment, 4, 5, and 6 represent the Medium copper treatment, and tanks 7, 8, and 9 contained specimens for the High copper treatment. Each tank underwent a complete water change on day 6 , indicated by the vertical line. 

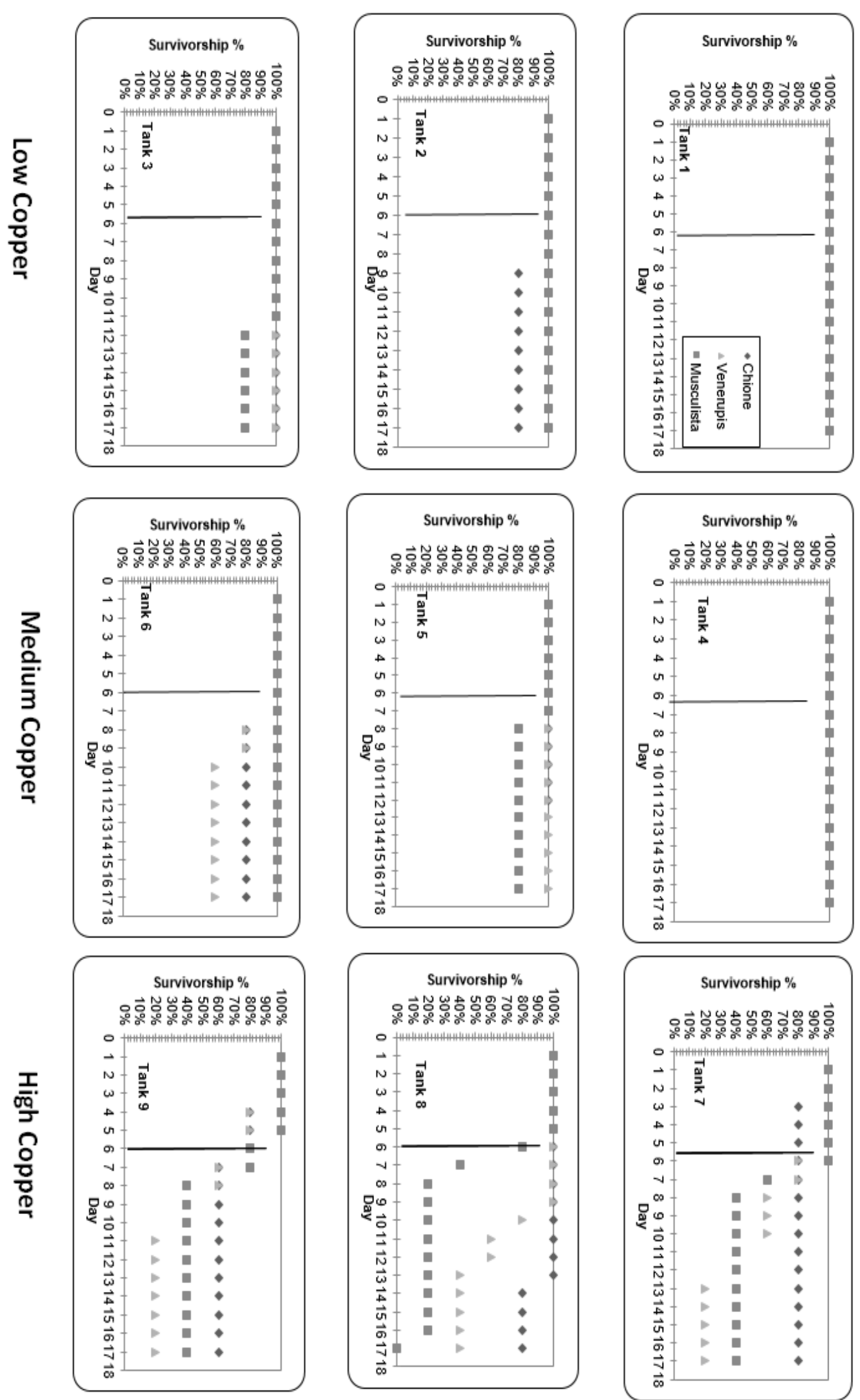
Figure 3.9. Survivorship percentage over time for each tank in the 12 day experiment, including all species at various estimated copper exposure levels. Tanks 1, 2, and 3 represent the Low copper treatment, 4, 5, and 6 represent the Medium copper treatment, and tanks 7, 8, and 9 contained specimens for the High copper treatment. Each tank underwent a complete water change on day 6 , indicated by the vertical line. 

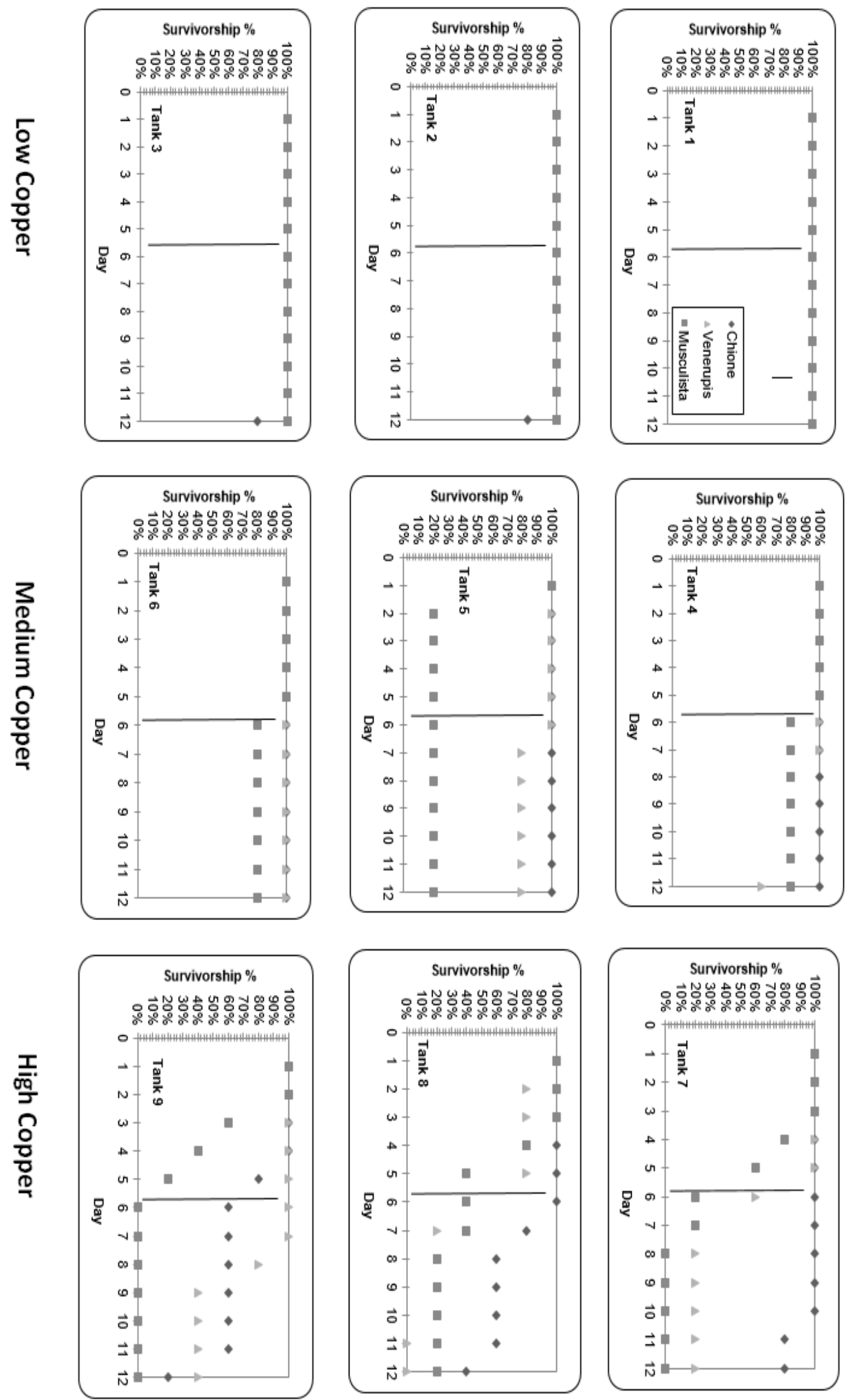


\subsubsection{Survivorship vs. Tissue Copper}

Survivorship was analyzed in relation to mean tissue copper accumulation for the whole body, gills, and digestive tract of individuals from each species (Appendix B, Tables 2.1, 3.1). Whole body and gill tissue copper accumulation showed generally negative trends across all three species for the 18 day experiments, and for Musculista in the 12 day exposure. Venerupis also showed a slight negative trend in survivorship for the 12 day exposure for whole body and gill tissue copper accumulation.

Survivorship data when plotted for the 18 day experiment visually showed a negative trend between the survivorship percentage and whole body copper concentration (Figure 3.6). However, closer examination of this trend showed no statistically significant relationship between survival percentage and whole body copper for the 18 day experiment. Regressions run for Chione $(p=0.079, F=65.0, D F=7$, $\left.R^{2}=0.985\right)$ and Venerupis $\left(p=0.073, F=76.22, D F=7, R^{2}=0.987\right)$ were fairly close to statistical significance, but Musculista $\left(p=0.251, F=5.787, D F=7, R^{2}=0.853\right.$ ) was not.

The trend in the 18 day experiment for whole body copper concentration was not observed in the 12 day experiment, as data within a species and exposure group in the 12 day experiment exhibited much higher variation (Figure 3.7). Statistical testing confirmed that there was no significant correlation between whole body copper and survival percentage for Chione ( $\mathrm{p}=0.546, \mathrm{~F}=0.749, \mathrm{DF}=7, \mathrm{R}^{2}=0.428$ ), Venerupis $\left(p=0.181, F=11.77, D F=7, R^{2}=0.922\right)$, and Musculista $(p=0.233, F=6.816, D F=7$, $\left.R^{2}=0.872\right)$.

The relationship between arcsine survivorship percentage and gill copper per dry mass for the 18 day experiments appeared to show a negative trend (Figure 3.6). 
However, linear regressions for each of the species [Chione $(p=0.108, F=33.9, D F=7$, $\left.R^{2}=0.971\right)$, Venerupis $\left(p=0.186, F=11.05, D F=7, R^{2}=0.917\right)$, Musculista $(p=0.088$, $\left.\left.F=51.18, D F=7, R^{2}=0.981\right)\right]$ did not show a statistically significant relationship between tissue copper and survival. Statistical significance was found in log-transformed gill copper per dry mass data for Chione $\left(p=0.0295, F=464.2, D F=7, R^{2}=0.998\right)$, but not Musculista $\left(p=0.220\right.$ and $\left.F=7.17, D F=7, R^{2}=0.885\right)$ or Venerupis $(p=0.115$, $\left.F=29.92, D F=7, R^{2}=0.967\right)$.

The trends observed in the 18 day experiment were not seen in the 12 day experiment (Figure 3.7). Statistical analysis of the 12 day data supported this perception, as neither Chione $\left(p=0.856, F=0.053, D F=7, R^{2}=0.050\right)$ nor Venerupis $\left(p=0.296, F=3.964, D F=7, R^{2}=0.799\right)$ showed a significant correlation between gill tissue copper and survival. There were not sufficient numbers of Musculista to test this relationship statistically.

Digestive tract copper accumulation only showed isolated trends with survivorship, with Venerupis showing a negative trend in the 18 day experiment, and Musculista showing a negative trend in the 12 day experiment for the Low and Medium copper treatments. No Musculista survived the High copper exposures in the 12 day experiment (Figures $3.6,3.7)$. For the 18 day experiment, there were no statistically significant relationships between survivorship and digestive tract copper accumulation for Chione $\left(p=0.831, F=0.0732, D F=7, R^{2}=0.069\right)$, Venerupis $(p=0.161, F=14.98$, $\left.D F=7, R^{2}=0.937\right)$ or Musculista $\left(p=0.594, F=0.548, D F=7, R^{2}=0.354\right)$. Similarly, the 12 day experiment also did not produce any significant results. Chione $(p=0.886$, 
$\left.F=0.032, D F=7, R^{2}=0.032\right)$ and Venerupis $\left(p=0.452, F=1.351, D F=7, R^{2}=0.575\right)$ both produced high $\mathrm{p}$ values, and Musculista did not have enough data to run a regression.

\subsubsection{Growth}

Growth measurements were converted to percentages based on the ratio between the final and initial width of each individual. Growth percentages were arcsine transformed for statistical testing. Means, medians, variances and standard deviations were examined based on growth percentage and overall growth (Appendix B, Table 4.1). Data were examined to compare copper concentration levels within and between species. Growth data for the 12 day experiment are presented here. Due to measurement errors, final widths in the 18 day experiment were shorter than initial widths; these data are not reported here.

\subsubsection{Growth in relation to copper exposure}

Very little difference was observed between growth in the Low copper treatment and the Medium and High copper treatments. This result may have stemmed from the short experimental period: all of the tested species showed a mean overall growth of less than $0.1 \mathrm{~mm}$ (Figure 4.1). The growth percentage showed a slight dip between Low and Medium copper treatments, but rose again for the High copper treatment in both Chione and Musculista, while Venerupis showed increases in growth for Medium and High copper treatments in comparison to the Low copper treatment (Figure 4.1). Statistical analysis of the data using a Shapiro-Wilk test indicated that the 
distribution of the arcsine transformed data was non-normal $\left(p=2.2 \times 10^{-16}\right)$. A KruskalWallis nonparametric test was used to analyze the growth data, and no significant differences were observed between the exposure groups in relation to growth $(p=0.652, F=0.857, D F=2)$, indicating no correlation between copper exposure and growth in this experiment.

\subsubsection{Growth in relation to species}

While there was no noticeable trend for growth in relation to copper concentration, there were some observed differences among species. In particular, Musculista exhibited a growth percentage more than double that of the next highest species, Chione (Figure 4.1). In terms of overall linear shell growth, Musculista and Chione were comparable, but the small initial size of Musculista led to a disparity in growth percentage that was statistically significant (Figure 4.1). A Kruskal-Wallis test was used to analyze the growth data, and there was a significant difference between the arcsine transformed growth percentages among species $\left(p=3.0 \times 10^{-5}, D F=2\right)$. Post hoc testing using a Dunn Test with Bonferroni correction revealed statistically significant differences between Musculista and the other two species ( $p=0.004$ for Chione and $p<0.001$ for Venerupis). There were no significant differences between Chione and Venerupis $(p=0.104)$. Examination of daily growth rate produced similar results among all three species (Appendix C), although Musculista showed the highest daily growth rate in all three copper exposures. A Shapiro-Wilk test showed that the distribution of the arcsine-transformed daily growth rate data was not normal $\left(p=2.60 \times 10^{-8}\right)$ and a 
Kruskal-Wallis test showed no significant differences among species $(p=0.421, D F=2)$ or copper concentrations ( $p=0.623, \mathrm{DF}=2)$.

\subsubsection{Growth in relation to tissue copper}

Growth percentage data from the 12 day exposure experiment were also plotted against tissue copper concentrations in the whole body, gills and digestive tract of selected individuals. Due to the small sample size of digested tissues and the fact that the growth percentages could not be individually linked to the digested samples, the means of the growth percentages were compared to mean tissue copper concentrations for each species.

Chione showed a decrease in growth percentage as whole body tissue copper increased, from a maximum growth percentage of $0.22 \%$ at tissue copper levels of 40.04 $\mu \mathrm{g} \mathrm{g}^{-1}$ to a low of $0.12 \%$ at tissue copper levels of $99.44 \mu \mathrm{g} \mathrm{g}^{-1}$ (Figure 4.2 , Figure 4.3 ). It should be noted that tissue copper levels did not match the expected trend based on exposure, with the highest tissue concentration occurring for Chione in the Medium copper treatment group. Overall, the trend was not significant for Chione, possibly due to a lack of samples for the High copper treatment as a result of too few surviving individuals.

The data for Musculista also were limited, although trends were visible in the relationship between growth and both gill and digestive copper concentrations. However, there are no gill copper data or digestive tract copper data for the High copper treatment group. Musculista showed a decrease from $0.51 \%$ growth at 18.53

$\mu \mathrm{g} \mathrm{g}^{-1}$ to $0.32 \%$ growth at $186.5 \mu \mathrm{g} \mathrm{g}^{-1}$ for gill copper, and a decrease from $0.51 \%$ at 20.22 $\mu \mathrm{g} \mathrm{g}^{-1}$ to $0.32 \%$ at $171.9 \mu \mathrm{g} \mathrm{g}^{-1}$ digestive tract copper. 
Figure 4.1. Measurements for growth percentage and overall growth for 12 day experiment. Error bars represent \pm one standard deviation. Refer to Appendix B, Table 4.1 for $\mathrm{N}$ values. The $\mathrm{p}$-values represent the statistically significant results of KruskalWallis testing, as the data distribution was not normal.

A.) Mean overall growth ( $\mathrm{mm}$ ) over a 12 day period for Chione californiensis, Venerupis philippinarum, and Musculista senhousia in Low, Medium and High copper treatments.

B.) Mean growth percentage over a 12 day period for Chione californiensis, Venerupis philippinarum, and Musculista senhousia in Low, Medium and High copper treatments. 


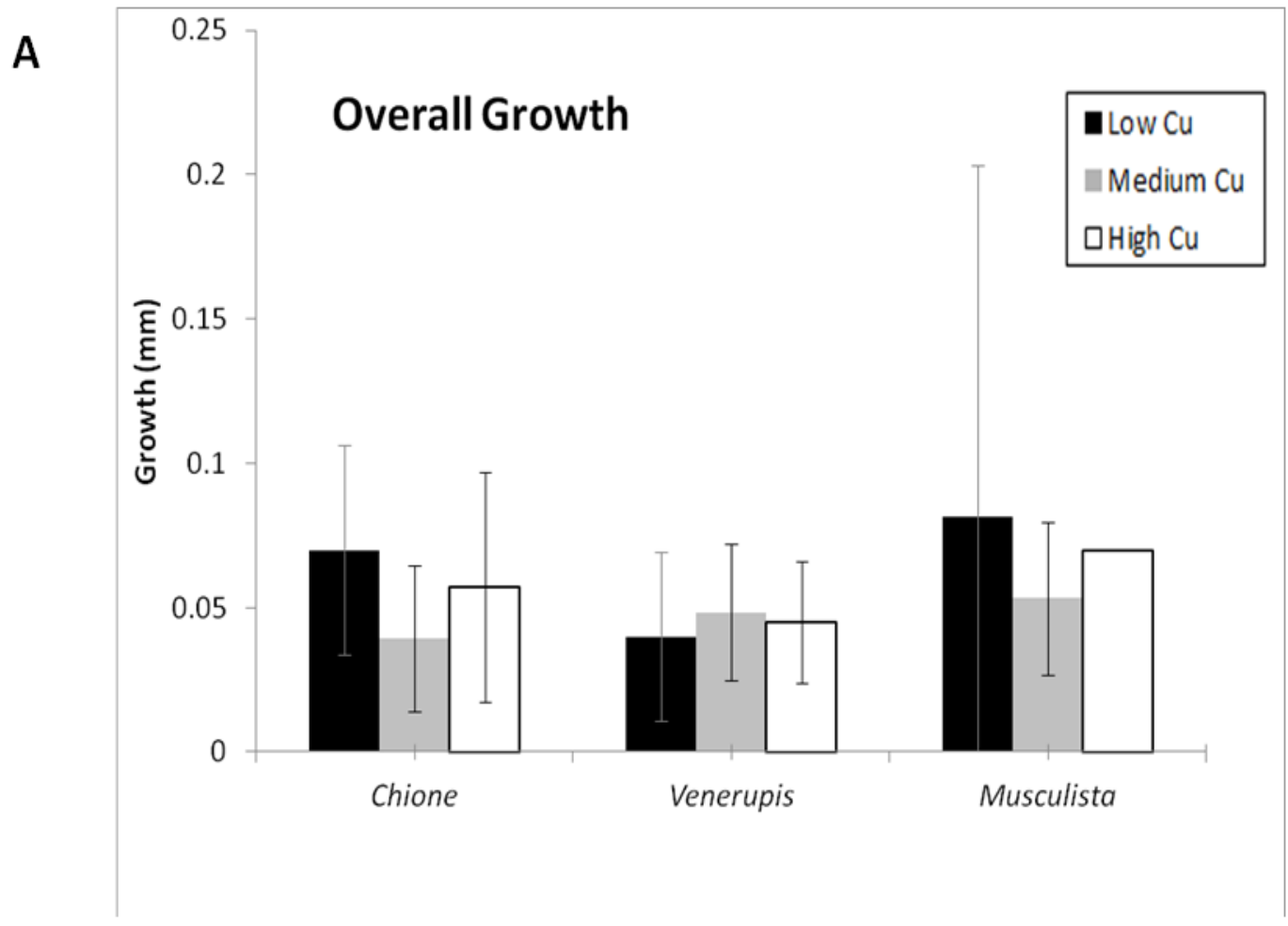

B

Growth Percentage

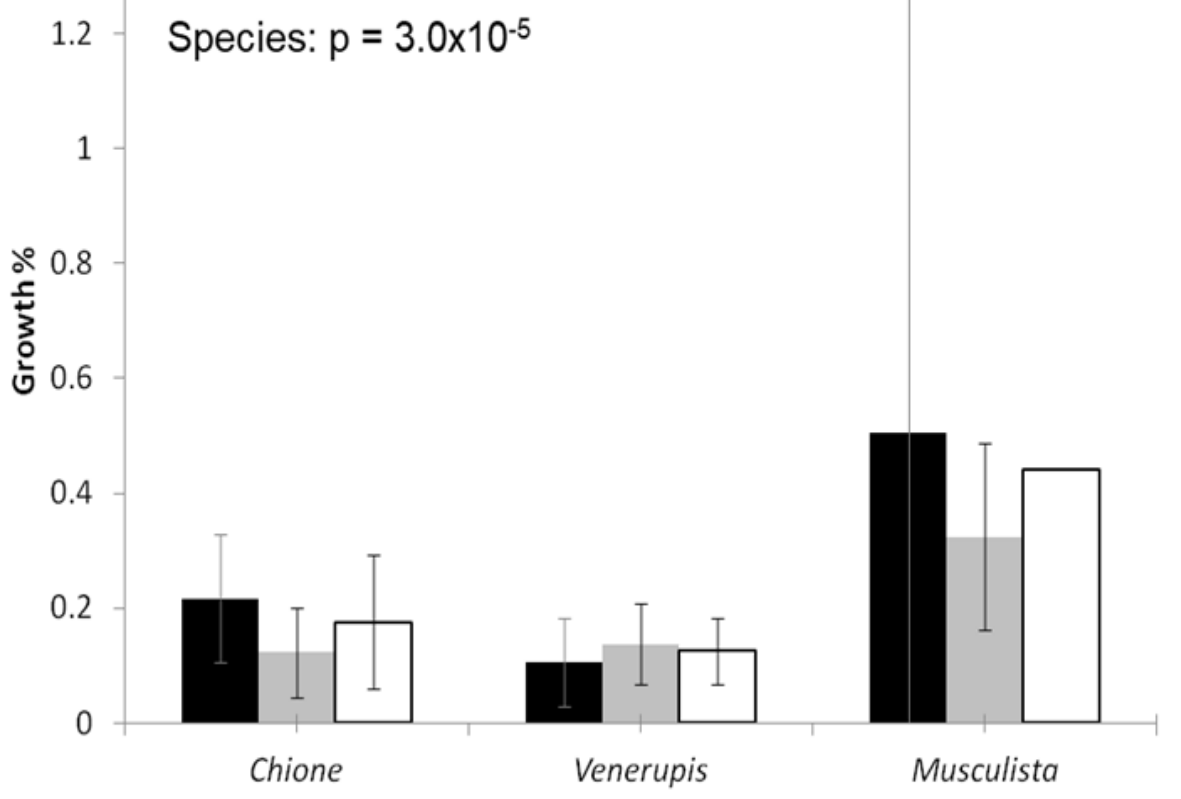


Figure 4.2. Mean growth percentage compared to mean copper per dry mass of whole body and dissected organs for specimens in the 12 day exposure experiment. Error bars represent \pm one standard deviation.
A.) Relationship between growth percentage and whole body copper per dry mass in $\mu \mathrm{g} \mathrm{g}^{-1}$ for Chione californiensis, Venerupis philippinarum, and Musculista senhousia.

B.) Relationship between growth percentage and gill copper per dry mass in $\mu \mathrm{g} \mathrm{g}^{-1}$ for Chione californiensis, Venerupis philippinarum, and Musculista senhousia.

C.) Relationship between growth percentage and digestive tract copper per dry mass in $\mu \mathrm{g} \mathrm{g}^{-1}$ for Chione californiensis, Venerupis philippinarum, and Musculista senhousia. 
A

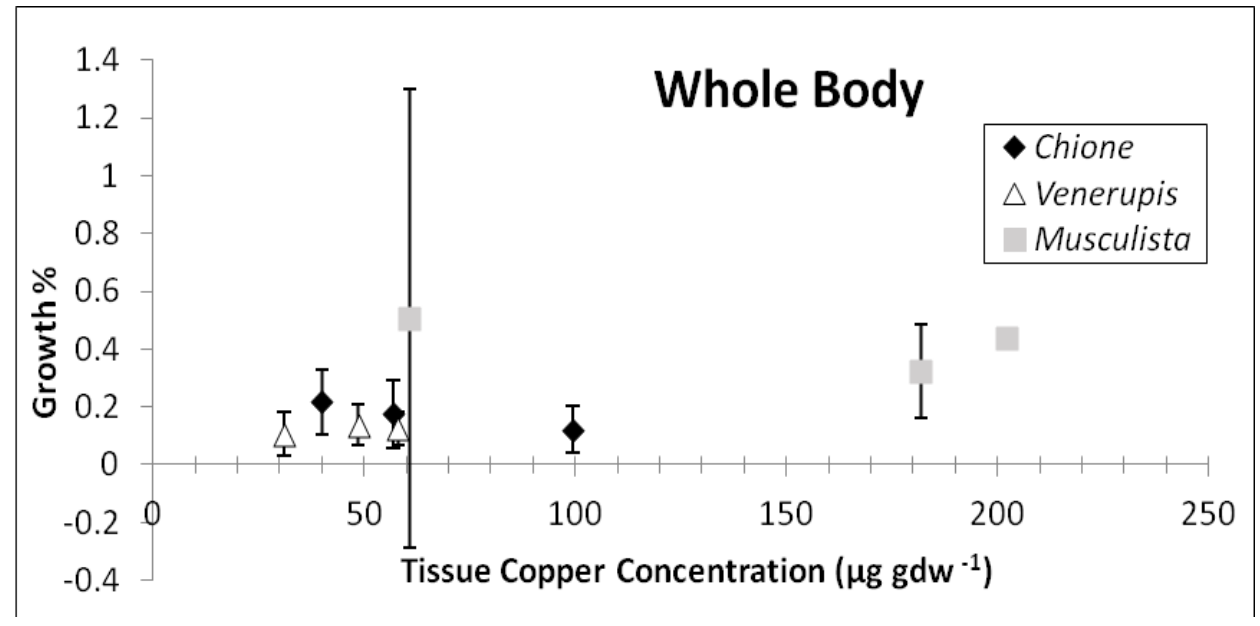

B
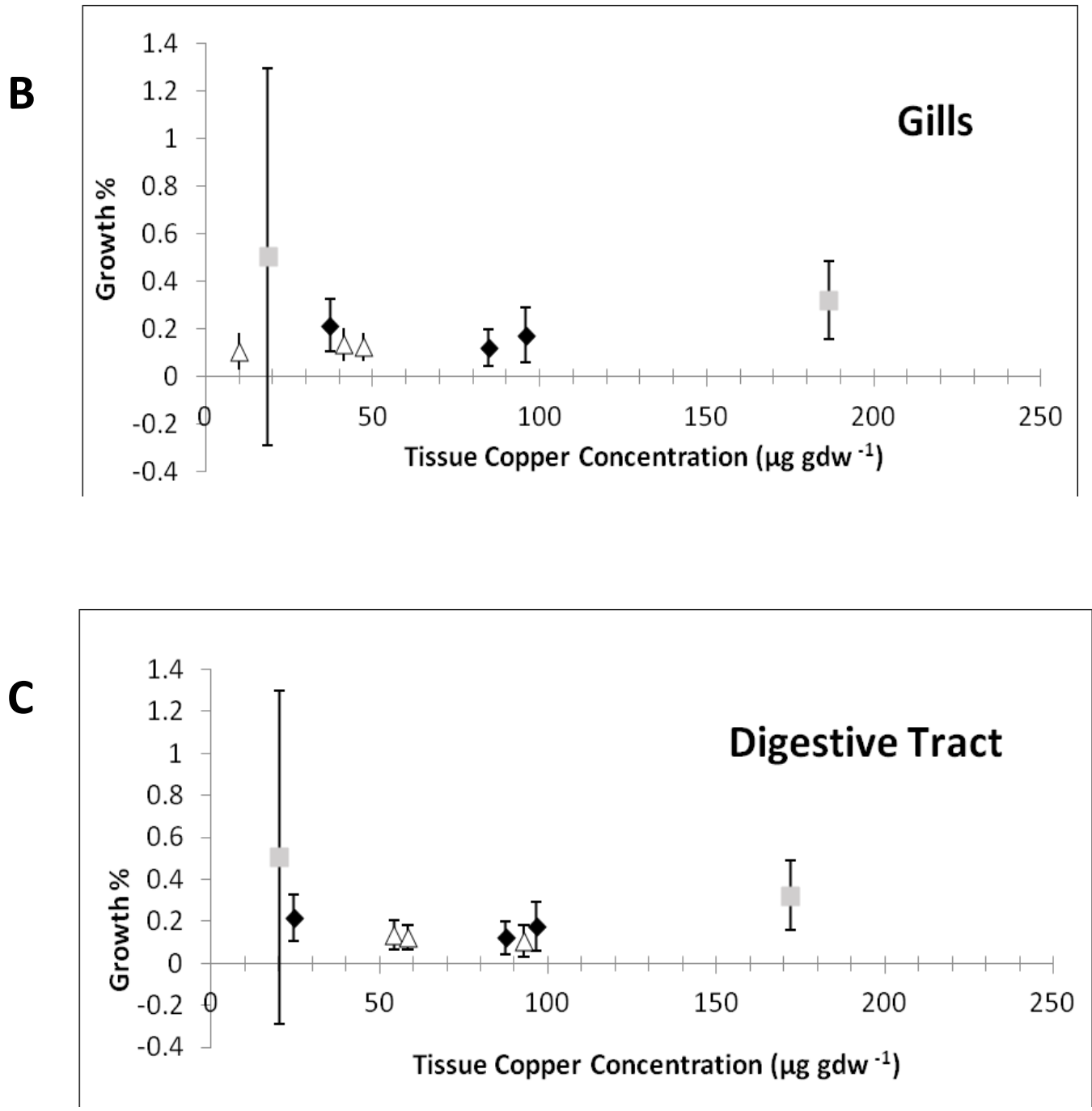
Figure 4.3. Relationship between growth percentage and mean copper per dry mass of whole body for specimens in the 12 day exposure experiment. Each data point represents growth data and whole body copper concentration for a single individual. Legend refers to species and copper concentration (e.g. Chione Medium represents the data for Chione in the Medium copper treatment group). 


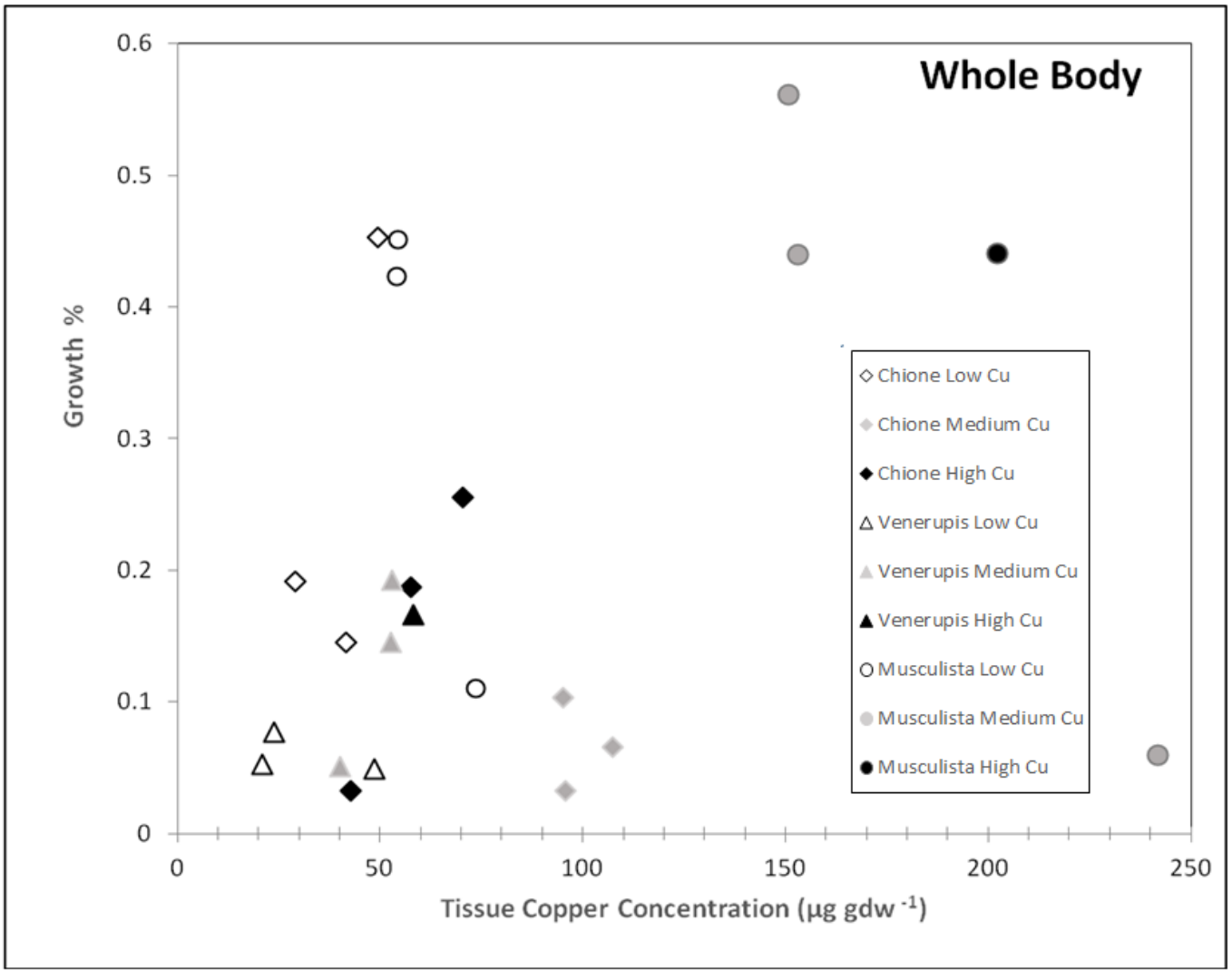


Examination of growth and whole body tissue copper concentration revealed no significant relationship between the two measures for all three species tested (Chione $p=0.117, F=29.19, D F=7, R^{2}=0.967$; Venerupis $p=0.209, F=8.59, D F=7, R^{2}=0.896$; Musculista $\mathrm{p}=0.930, \mathrm{~F}=0.012, \mathrm{DF}=7, \mathrm{R}^{2}=0.012$ ). Gill copper accumulation also did not show any statistical significance (Chione $p=0.494, F=1.036, D F=7, R^{2}=0.509$; Venerupis $\left.\mathrm{p}=0.209, \mathrm{~F}=8.59, \mathrm{DF}=7, \mathrm{R}^{2}=0.896\right)$, and similarly for digestive tract copper concentration (Chione $\mathrm{p}=0.464, \mathrm{~F}=1.255, \mathrm{DF}=7, \mathrm{R}^{2}=0.557$; Venerupis $\mathrm{p}=0.053$, $\left.F=141.8, D F=7, R^{2}=0.993\right)$, though Venerupis was close to statistical significance. There were not enough data for Musculista to perform a regression for either gill or digestive tract copper concentration.

\subsubsection{Mucus production}

Mucus production was variable within the various exposure groups. Individuals of the same species within the same exposure groups showed drastically different levels of mucus production; by contrast, body size as reflected in tissue dry mass was much more consistent. This situation led to a large standard deviation within the mucus/dry mass ratios for each group, since the variation in mucus production level was high (Appendix B, Table 5.1). The large standard deviation within mucus/dry mass ratios was particularly true for the 18 day exposure.

In addition to this high variation, different trends were observed between the two exposure periods as well (Appendix B, Table 5.1). For the 18 day exposure, all three species exhibited the lowest mucus production at the Medium copper treatment, and the highest mucus/dry mass ratios in the High copper treatment group (Figure 5.1). 
Figure 5.1. Mucus/Dry Mass ratio for Chione californiensis, Venerupis philippinarum, and Musculista senhousia. Ratios were calculated by dividing the dried mass of mucus collected over a 30 minute period by the dried whole body mass of the specimen from which the mucus was collected. Error bars represent \pm one standard deviation. $\mathrm{N}$ values are reported in Appendix B, Table 5.1. The p-values represent the statistically significant results of Kruskal-Wallis testing, as the data distribution was not normal.

A. 18 day exposure experiment Mucus/Dry Mass ratios for all species in the Low, Medium and High copper treatment groups, collected at the end of an 18 day exposure period.

B. 12 day exposure experiment Mucus/Dry Mass ratios for all species in the Low, Medium and High copper treatment groups, collected at the end of a 12 day exposure period. 
A

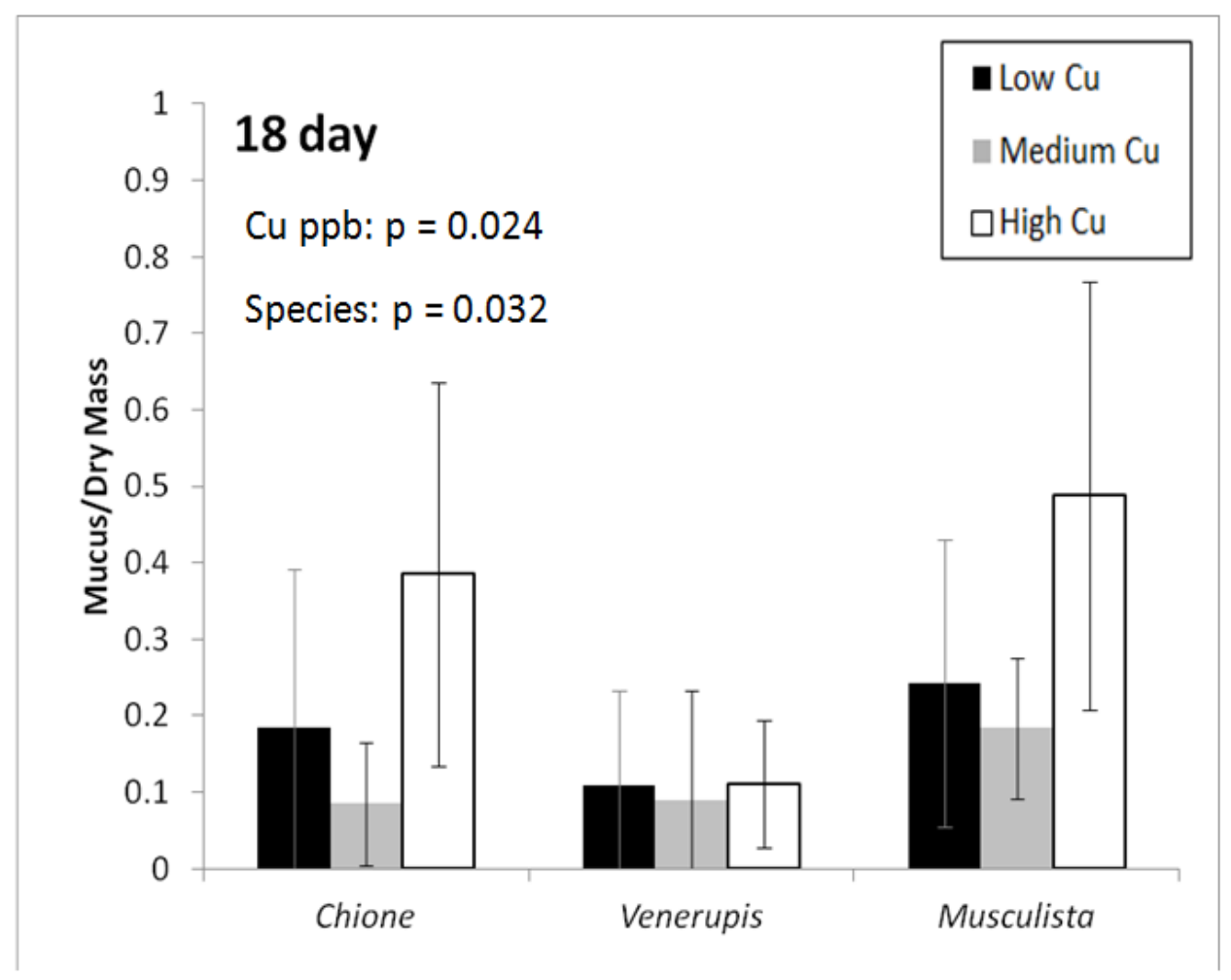

B

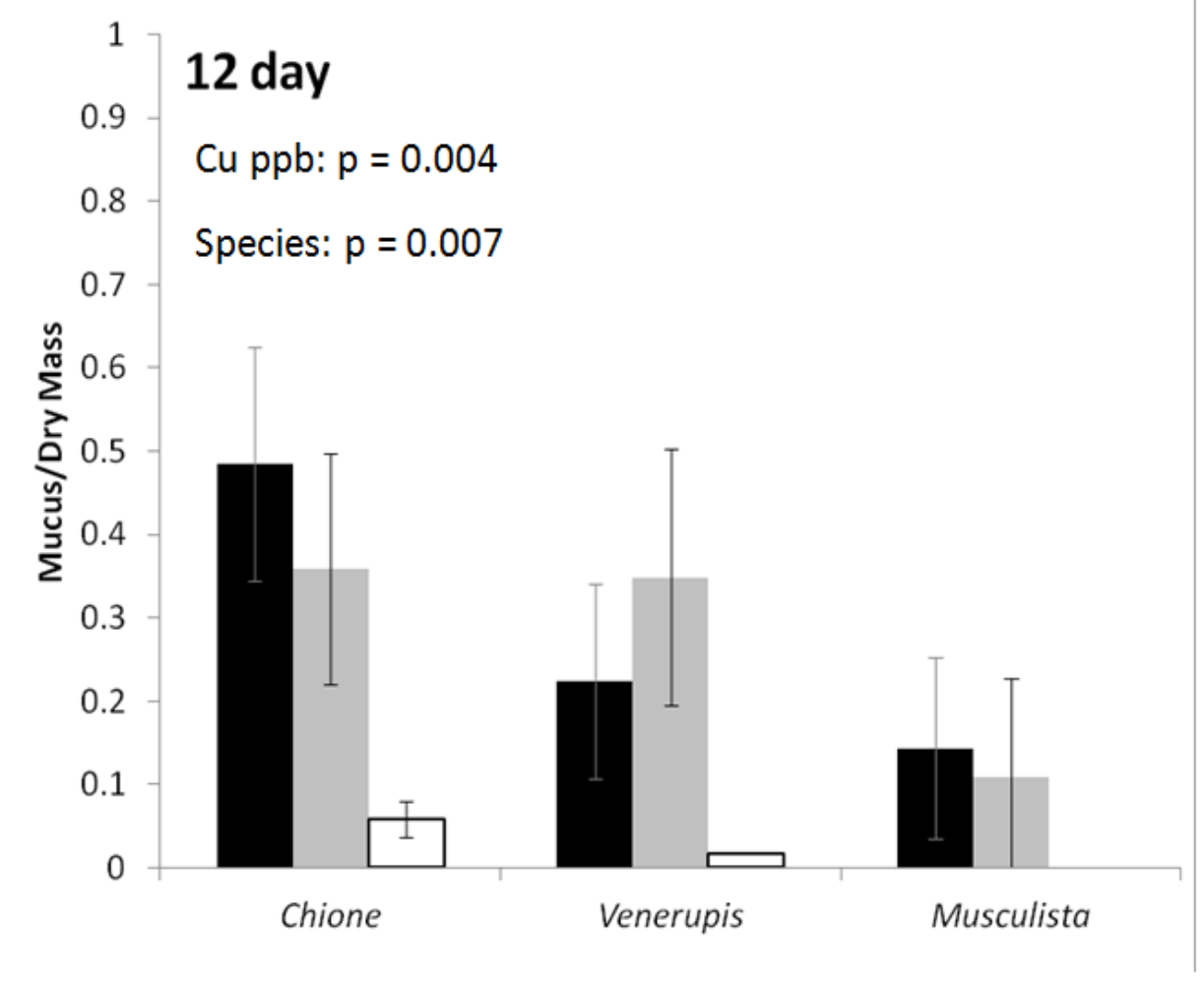


The 12 day exposure, however, showed very different results. Mucus production decreased as copper levels increased for Chione and Musculista, and was lowest for Venerupis in the High copper treatment group (Figure 5.1). This is contrary to the data from the 18 day experiment, which showed the highest mucus production levels in the High copper treatment for all species. It should be noted that the data, particularly for the Venerupis and Musculista are more limited for the 12 day experiment than the 18 day experiment, especially in the High copper treatment, where there was only one survivor each for Musculista and Venerupis, in comparison to two survivors for each species in the 18 day experiment.

Mucus production didn't show any consistent trends within or among species, or between experiments. Mucus/dry mass ratios were generally higher in the 12 day experiment for the Low and Medium copper treatment groups but lower for the High copper treatment group compared to the 18 day experiment (Figure 5.1).

Statistical analysis of mucus production to dry weight ratios was performed separately for the 18 day and 12 day experiments. The data were arcsine transformed and analyzed for normality. The Shapiro-Wilk test indicated that the distributions in both experiments were not normal $\left(p=5.77 \times 10^{-6}\right.$ and 0.021 for the 18 and 12 day experiments, respectively).

A Kruskal-Wallis test on mucus production to dry weight ratios for the 18 day experiment showed significant $p$-values for both species and copper concentration ( $p=0.032, D F=2$ and $p=0.024, D F=2$, respectively). Venerupis exhibited the lowest mucus/dry mass ratio, while Musculista showed the highest ratio (Figure 5.1). A post hoc Dunn test with Bonferroni correction showed that Venerupis and Musculista were 
statistically significantly different in mucus/dry mass $(p=0.010)$, while there was no significant difference between Chione and Musculista or Venerupis $(p=0.406$ and $p=0.126$, respectively). Mucus production to dry mass ratios in the High copper treatment were significantly higher than the in both the Low and Medium copper treatments across species ( $p=0.016$ and $p=0.046$, respectively). No significant difference was observed between the Low and Medium treatments $(p=0.900)$.

The 12 day experiment also produced statistically significant results, but trends were different compared to the 18 day experiment. Kruskal-Wallis tests for the 12 day experiment produced significant $p$-values for the difference between species $(p=0.007$, $\mathrm{DF}=2)$ and copper concentrations $(p=0.004, \mathrm{DF}=2)$. This time, post hoc Dunn tests with Bonferroni corrections revealed statistically significant differences between Chione and Musculista $(p=0.003)$ but not between Venerupis and Musculista $(p=0.053)$ or Chione and Venerupis $(p=0.515)$. There also were significant differences between the High copper treatment group and both the Low and Medium copper treatment groups $(p=0.003$ and $p=0.002)$, but not between the Low and Medium copper treatment groups $(p=1.000)$.

Whereas Musculista produced the highest mucus/dry mass ratios in the 18 day exposure, the mucus/dry mass ratio for Musculista in the 12 day experiment were the lowest among the three species. In terms of copper exposures, the High copper treatments showed significantly lower mucus/dry mass ratios, unlike bivalves exposed to this copper concentration in the 18 day experiment. 


\subsubsection{Mucus production in relation to tissue copper}

Mucus production patterns were examined at the individual and treatment levels. Individuals that were tested for mucus production were then digested for whole body copper tissue analysis. Thus, mucus production could be examined in relation to copper tissue concentrations for individual animals. Mucus production also was examined by grouping individuals within a treatment and comparing mean mucus production rates with mean tissue copper concentrations.

In the 18 day experiment, mean pooled mucus/dry mass ratios seemed to increase with increasing whole body copper concentration for Chione and Musculista, although each showed a decrease at the Medium copper exposure (Figure 5.2). A linear regression was used to examine if there was a statistical relationship between mucus/dry mass ratio and whole body copper accumulation. No significant relationship was evident for Chione $\left(p=0.534, F=0.801, D F=7, R^{2}=0.447\right)$, Venerupis $(p=0.717$, $\left.F=0.228, D F=7, R^{2}=0.186\right)$, or Musculista $\left(p=0.330, F=3.063, D F=7, R^{2}=0.754\right)$.

In the 12 day experiment, mean mucus/dry mass ratios did not show any apparent trends in relation to whole body copper concentration (Figure 5.3). Similarly to the 18 day experiment, linear regressions showed no indication of a significant relationship between transformed mucus/dry mass ratio and whole body tissue copper per dry mass for any of the species (Chione $\mathrm{p}=0.956, \mathrm{~F}=0.004, \mathrm{DF}=7, \mathrm{R}^{2}=0.004$; Venerupis $\mathrm{p}=0.631, \mathrm{~F}=0.428, \mathrm{DF}=7, \mathrm{R}^{2}=0.300$; Musculista $\mathrm{p}=0.482, \mathrm{~F}=1.121$, $\mathrm{DF}=7, \mathrm{R}^{2}=0.529$ ).

Mucus/dry mass ratio was also compared with copper accumulation for both gill tissue and digestive tract tissue. However, gill and digestive tract tissue were taken from 
specimens not used in mucus collection. Thus, gill and digestive tract copper concentrations were pooled by species and treatment and tested against the same mucus/dry mass ratios as whole body copper. There were no mucus/dry mass ratios for the specimens from which gill and digestive tract tissue were collected.

Mucus/dry mass ratio appeared to increase with increasing gill copper concentration in the 18 day experiment for Chione and Musculista, but exhibited a similar dip in the Medium copper treatment to that seen for whole body copper (Figure 5.1). A linear regression revealed no statistically significant relationship between mucus/dry mass ratio and gill copper accumulation for any of the species (Chione $p=0.347, F=2.714, D F=7, R^{2}=0.731 ;$ Venerupis $p=0.458, F=1.303, D F=7, R^{2}=0.036$; Musculista $\left.\mathrm{p}=0.168, \mathrm{~F}=13.68, \mathrm{DF}=7, \mathrm{R}^{2}=0.932\right)$.

The 12 day experiment did not show any apparent trends between gill copper accumulation and mucus/dry mass ratios. Linear regressions supported this observation (Chione $\mathrm{p}=0.357, \mathrm{~F}=2.527, \mathrm{DF}=7, \mathrm{R}^{2}=0.716$; Venerupis $\mathrm{p}=0.747, \mathrm{~F}=0.177, \mathrm{DF}=7$, $\left.R^{2}=0.150\right)$. There were too few surviving Musculista to perform a regression.

The digestive tract data for both experiments showed no apparent trends. Linear regressions to examine potential relationships between mucus/dry mass ratio and digestive tract copper concentration for the 18 day experiment showed no significant results (Chione $\mathrm{p}=0.713, \mathrm{~F}=0.234, \mathrm{DF}=7, \mathrm{R}^{2}=0.189$; Venerupis $\mathrm{p}=0.805$, $F=0.100, D F=7, R^{2}=0.091 ;$ Musculista $\left.p=0.515, F=0.912, D F=7, R^{2}=0.477\right)$.

The 12 day experiment digestive tract data also did not show any apparent relationship between mucus/dry mass ratio and digestive tract copper per dry mass for any of the species. Linear regressions supported this observation (Chione $p=0.388$, 
$F=2.054, D F=7, R^{2}=0.673 ;$ Venerupis $p=0.788, F=0.024, D F=7, R^{2}=0.023$ ). Too few Musculista survived the experimental period, and linear regressions could not be performed.

Mucus/dry mass ratios for individual bivalves were compared to whole body copper concentrations for the same individuals. Unlike the pooled mean ratios described above, data from both experiments did not show a strong correlation between mucus/body mass ratio and whole body copper concentration (Figure 5.4). A linear regression for both experiments did not show a significant relationship between whole body copper accumulation and mucus/dry mass ratio for the larger data set (Chione $\mathrm{p}=0.241, \mathrm{~F}=1.645, \mathrm{DF}=7^{\prime}$ Venerupis $\mathrm{p}=0.317, \mathrm{~F}=1.192, \mathrm{DF}=7$; Musculista $p=0.606, F=0.302, D F=7)$. The 12 day experiment similarly showed no statistically significant relationship between whole body copper accumulation and mucus/dry mass ratio, nor were there any apparent trends (Chione $\mathrm{p}=0.868, \mathrm{~F}=0.030, \mathrm{DF}=7$; Venerupis $p=0.683, F=0.187, D F=7 ;$ Musculista $p=0.501, F=0.526, D F=7)$. This larger data set indicates that mucus/dry mass ratios do not show significant correlations with whole body copper accumulation, and supports the results of linear regressions on the smaller data set of mean mucus/dry mass ratio and mean whole body copper accumulation. 
Figure 5.2. Mean mucus/dry mass ratio compared to mean copper per dry mass of whole body and dissected organs for specimens in the 18 day exposure experiment. Error bars represent \pm one standard deviation. Refer to Appendix B, Table 5.1 for $\mathrm{N}$ values

A.) Whole body tissue

B.) Gill tissue

C.) Digestive tract tissue 

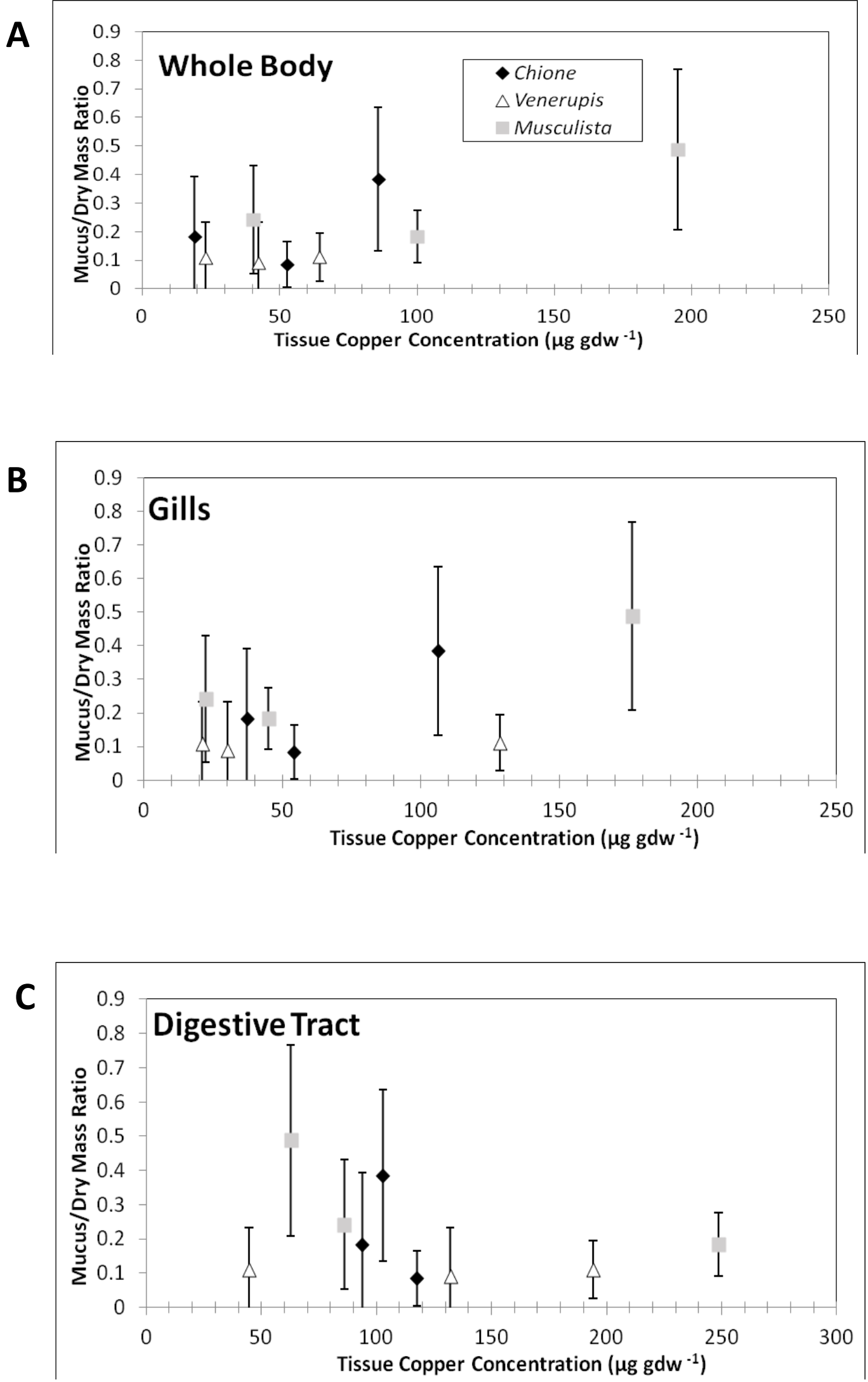
Figure 5.3. Mean mucus/dry mass ratio compared to mean copper per dry mass of whole body and dissected organs for specimens in the 12 day exposure experiment Mean mucus/dry mass ratio of Chione californiensis, Venerupis philippinarum, and Musculista senhousia compared to mean copper per dry mass for specimens in the 12 day exposure experiment. Error bars represent \pm one standard deviation. Refer to Appendix $\mathrm{B}$, Table 5.1 for $\mathrm{N}$ values.
A.) Whole body tissue
B.) Gill tissue
C.) Digestive tract tissue 


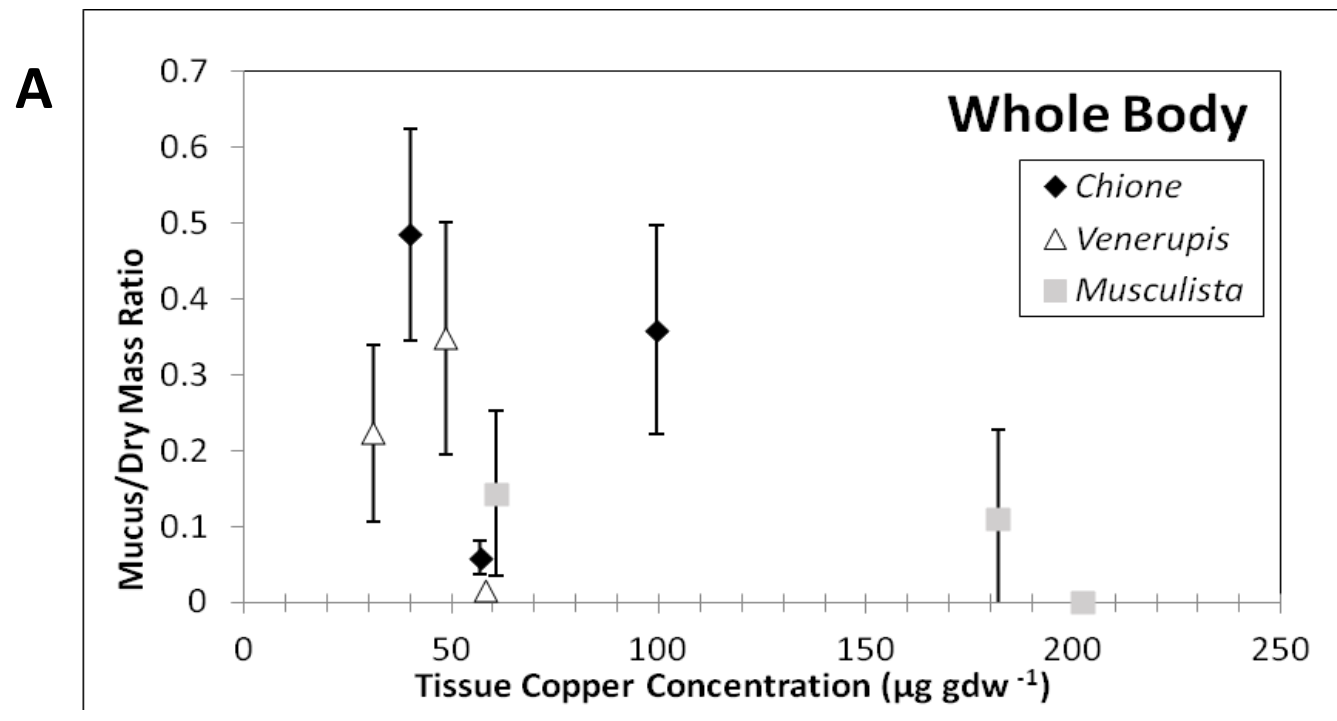

B

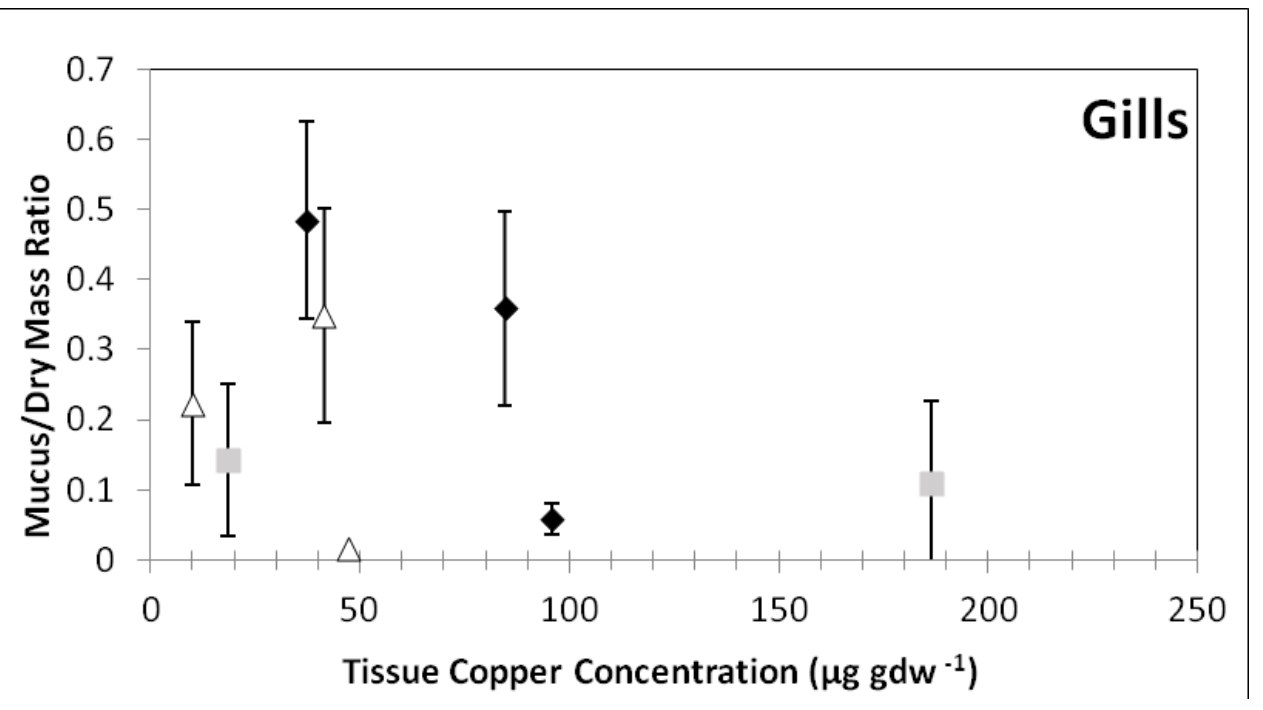

C

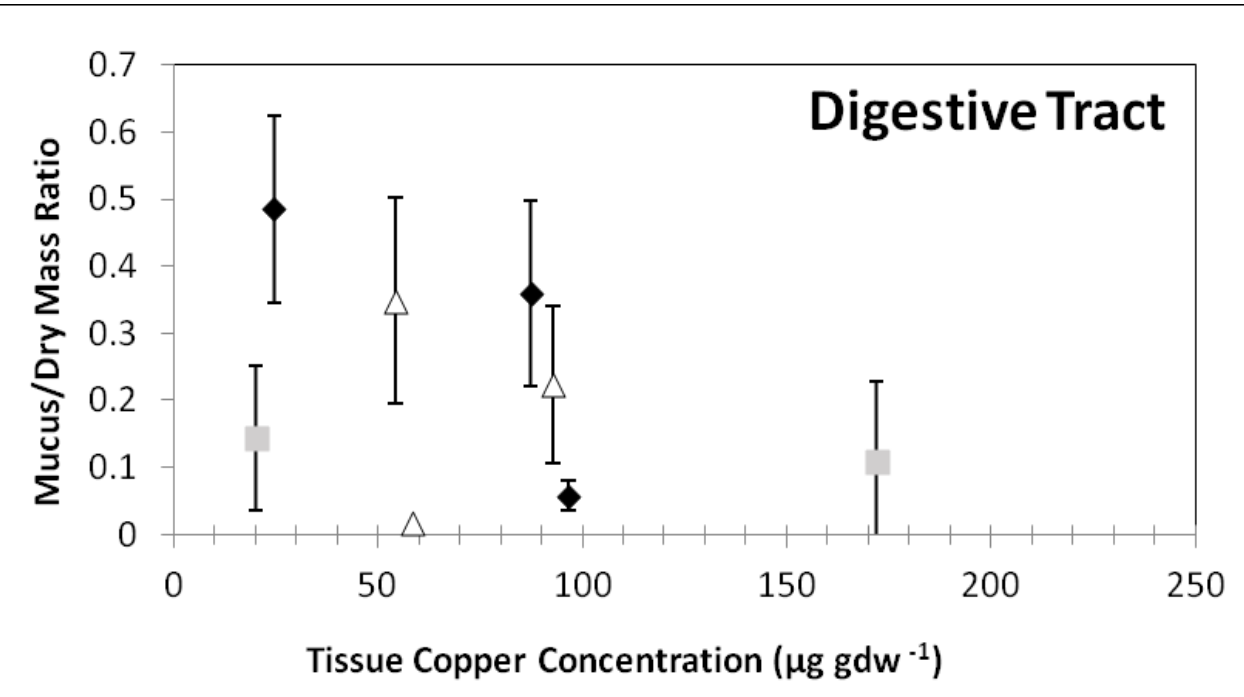


Figure 5.4. Mucus/dry mass ratio compared to copper per dry mass of whole body for the 18 day and the 12 day exposure experiments. Relationship between mucus/dry mass ratio of Chione californiensis, Venerupis philippinarum, and Musculista senhousia and copper per dry mass of whole body tissue. Each point represents an individual animal.

A.) 18 day experiment

B.) 12 day experiment 


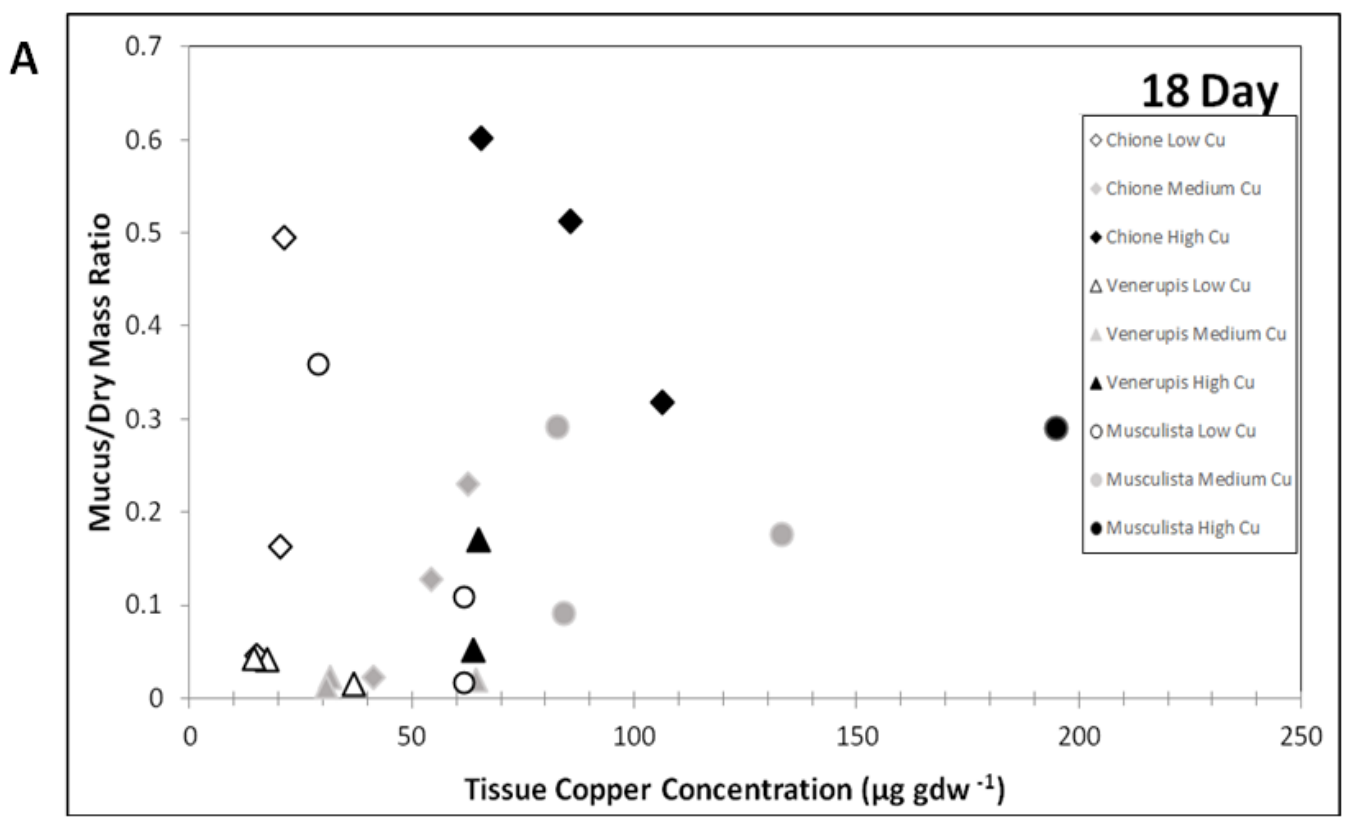

B

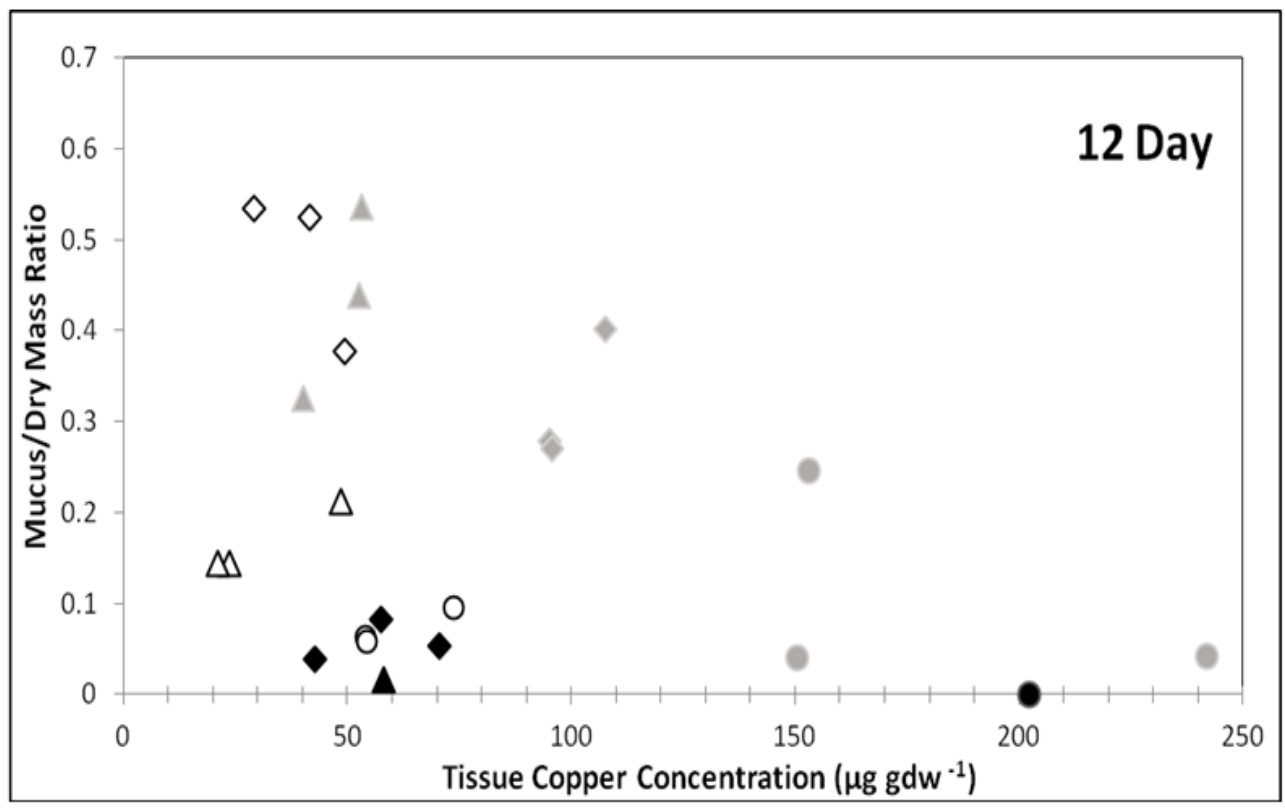




\subsubsection{Algae Depletion}

Algae depletion data were analyzed in three ways: measured depletion rates; depletion rates in Low, Medium and High copper treatment groups, with simultaneous results from an empty test chamber (control) subtracted; and depletion rates in Medium and High copper treatment groups, with simultaneous depletion rates in the Low copper treatment group subtracted.

\subsubsection{Algae Depletion - Measured Rates}

Musculista exhibited the lowest measured depletion rates among the three species across both experiments and the majority of the exposure groups, often approaching depletion rates close to the control (Appendix B, Table 6.1). Rates for Musculista were similar to those of the control group across both experiments, in most treatment groups.

Most of the other trends observed in the measured algae depletion data are not exhibited across both experiments or require the elimination of outliers. In some treatments, measured cell depletion rates increased over time: Venerupis and Chione in the Medium copper treatment groups during the 18 day experiment and Venerupis in the High copper treatment group when ignoring Day 0 as an outlier (Figure 6.1A). However, these same trends were not observed in the 12 day experiment. Chione also showed some slight trends in the Low copper treatment groups in both experiments, with a slight positive correlation with time in the 18 day experiment and a slight negative correlation in the 12 day experiment (Figures 6.1A, 6.1B). 


\subsubsection{Algae Depletion Rates - Control Subtracted}

In the pilot study, the control showed some algal depletion, and to correct for this effect, the control depletion rates were subtracted from measured depletion rates. In the control-subtracted data, there was an apparent increase over time in cell depletion rates for Venerupis philippinarum in both the Medium and High copper treatments (Figures 6.1, 6.2). However, this trend was not clearly observed in the 12 day experiment (Figures 6.3, 6.4). The Low copper treatment group with Chione showed trends for both experiments; however, the trends were reversed: the 18 day experiment showed a decrease in algae depletion over time, while the 12 day experiment showed an increase. Though there were some similarities in the data observed between the two experiments, these similarities were not consistent over species or copper concentration and did not constitute a sustained trend. Most similarities between the two experiments generally did not show any sustained trends (Figures 6.1, 6.2, 6.3, 6.4).

\subsubsection{Algae Depletion Rates - Low Copper Treatment Subtracted}

To examine the effects of elevated copper concentrations on bivalve feeding rates, algae depletion rates for bivalves in the Low copper treatment group for each species were subtracted from rates for Medium and High copper treatment groups for the same species. Unlike the subtraction of depletion rates from the control chambers, subtracting depletion rates for the Low copper treatment groups had a noticeable impact on the calculated depletion rates. Some trends were especially evident, such as the significant positive correlation $(p=0.005)$ between depletion and time seen in the Medium copper treatment of Venerupis in the 18 day exposure (Figures 6.5B, 6.6B). A 
similar correlation was observed in the High copper treatment group of Chione $(p=$ 0.054 ) in the 12 day exposure (Figures 6.7A, 6.8A). Similar trends were not apparent in the other species or treatment groups. 
Figure 6.1. Control subtraction representation of mean algae depletion rates for 18 day experiment by species. Mean total algae depletion rates with control subtracted for Chione californiensis, Venerupis philippinarum, and Musculista senhousia over 18 days in Low, Medium, and High copper treatments. Algae depletion rates in control chambers were subtracted from the depletion rates measured in chambers containing bivalves. Error bars represent \pm one standard deviation
A.) Chione californiensis
B.) Venerupis philippinarum
C.) Musculista senhousia 
A

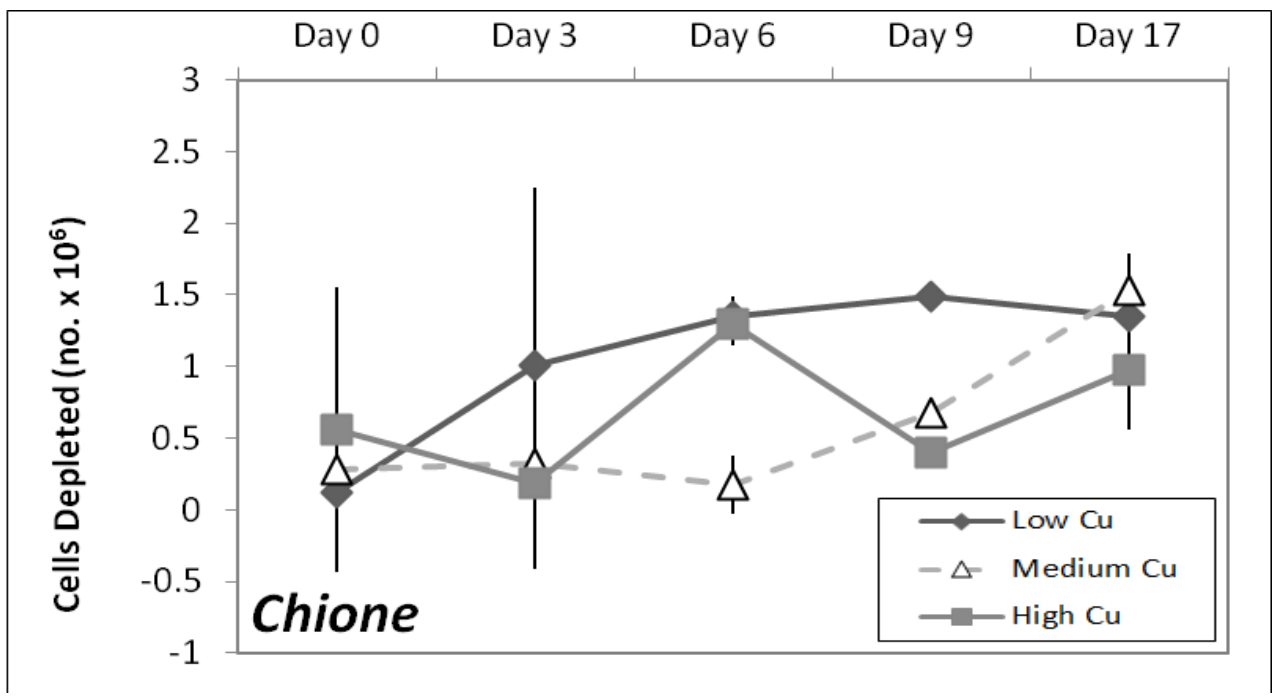

B
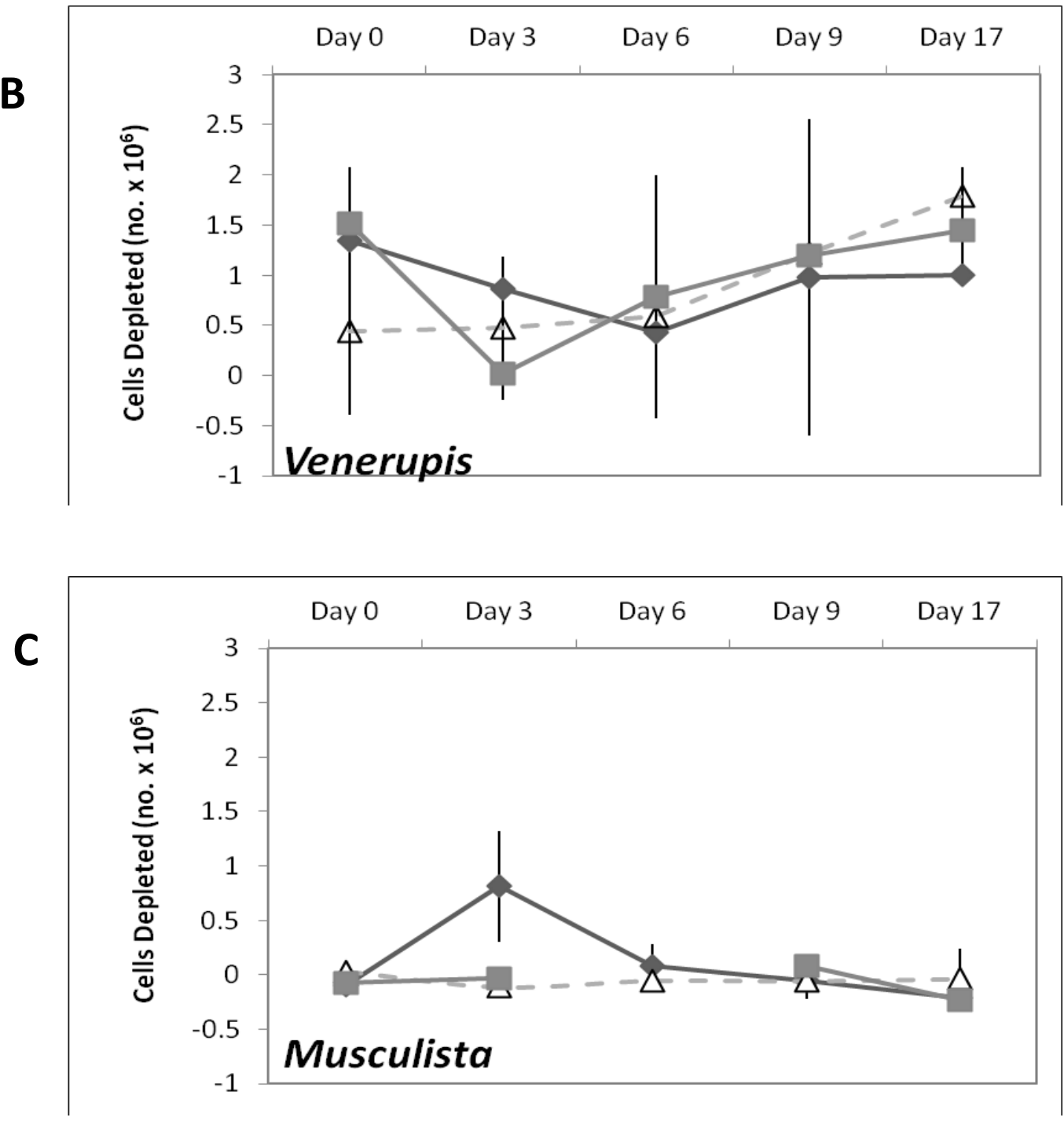
Figure 6.2. Control subtraction representation of mean algae depletion rates for 18 day experiment by concentration. Mean total algae depletion rates with control subtracted for Chione californiensis, Venerupis philippinarum, and Musculista senhousia over 18 days in Low, Medium, and High copper treatments. Algae depletion rates in control chambers were subtracted from the depletion rates measured in chambers containing bivalves. Error bars represent \pm one standard deviation.
A.) Low copper treatment

B.) Medium copper treatment

C.) High copper treatment 
A

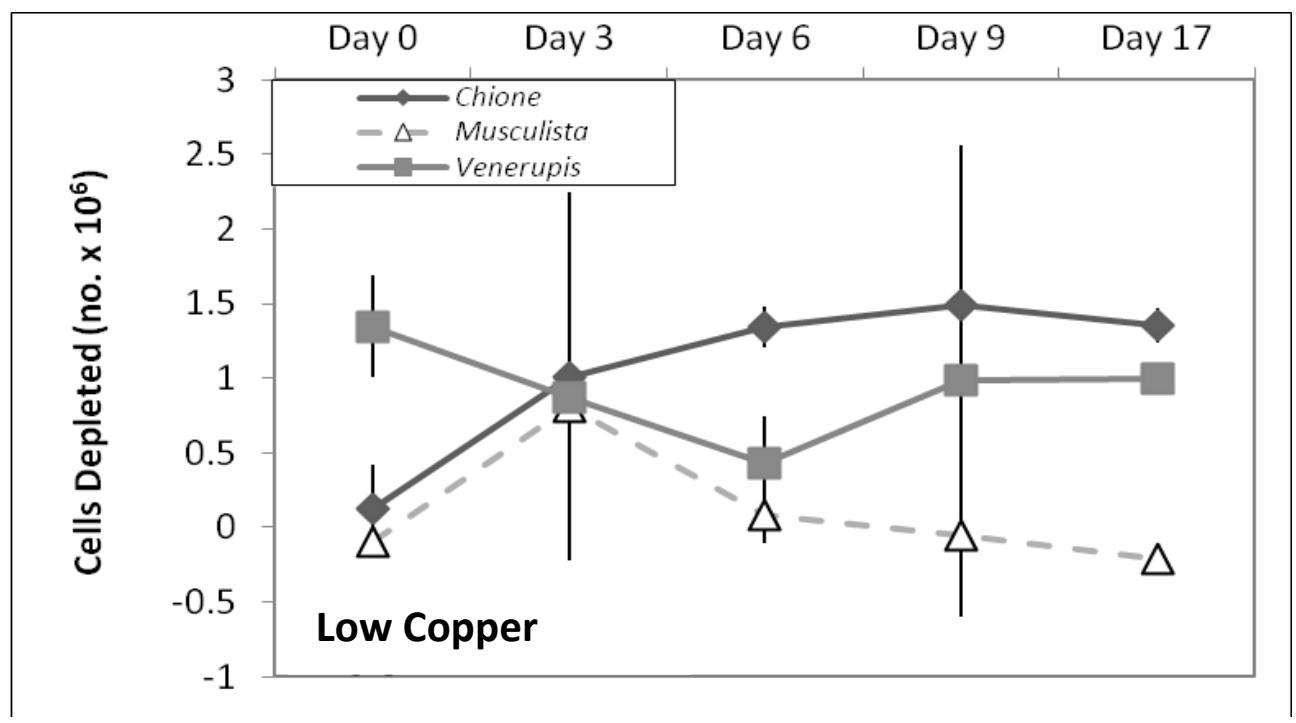

B

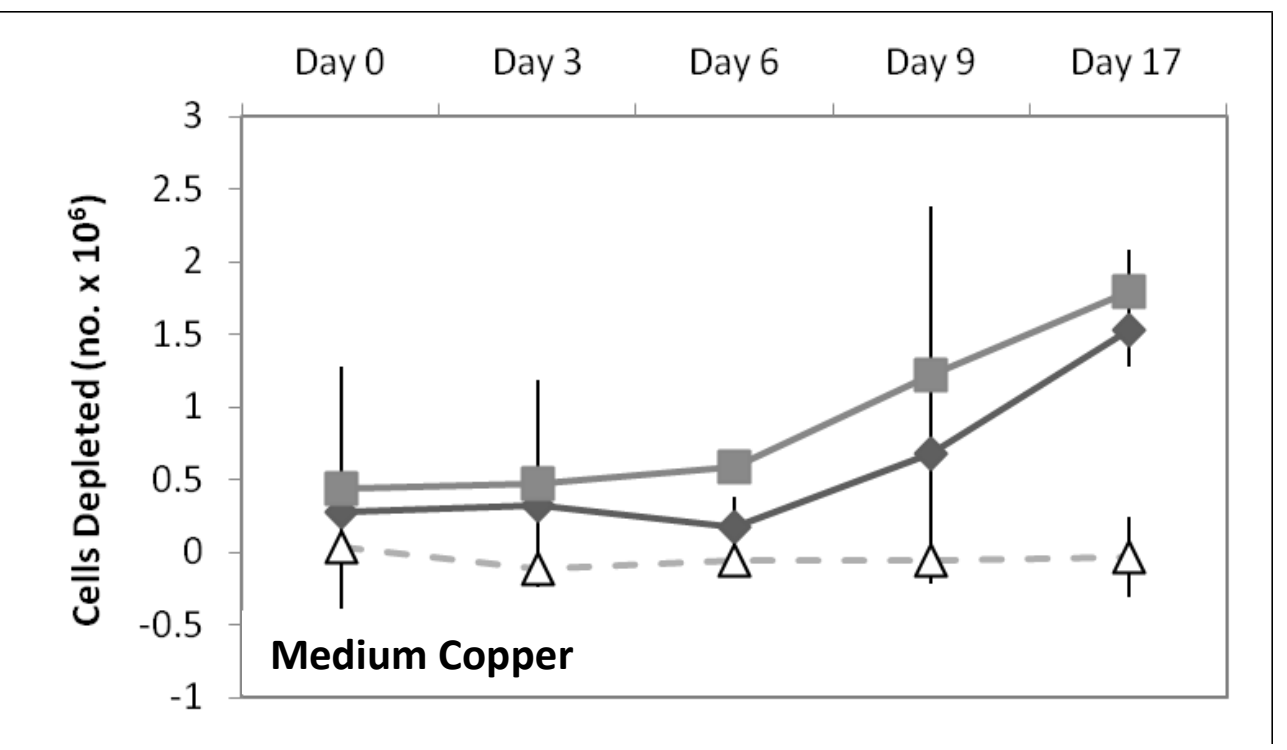

C

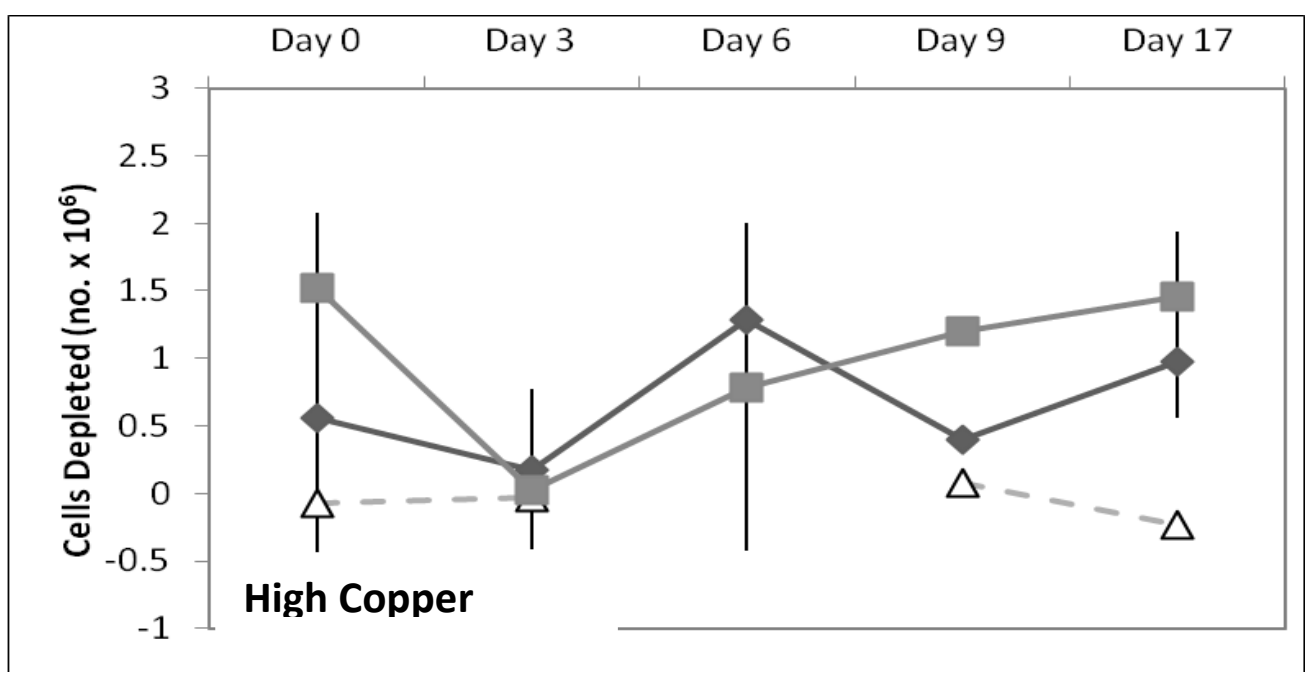


Figure 6.3. Control subtraction representation of mean algae depletion rates for 12 day experiment by species. Mean total algae depletion rates with control subtracted for Chione californiensis, Venerupis philippinarum, and Musculista senhousia over 12 days in Low, Medium, and High copper treatments. Algae depletion rates in control chambers were subtracted from the depletion rates measured in chambers containing bivalves. Error bars represent \pm one standard deviation.

A.) Chione californiensis

B.) Venerupis philippinarum

C.) Musculista senhousia 


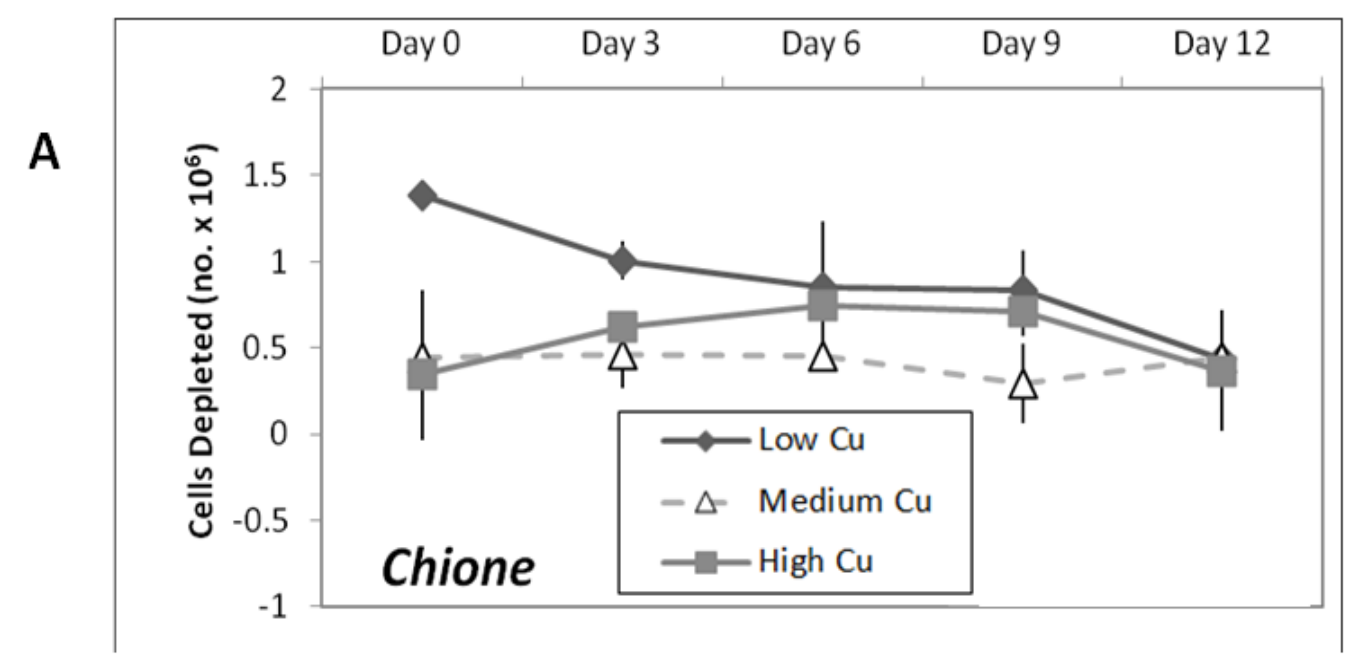

B
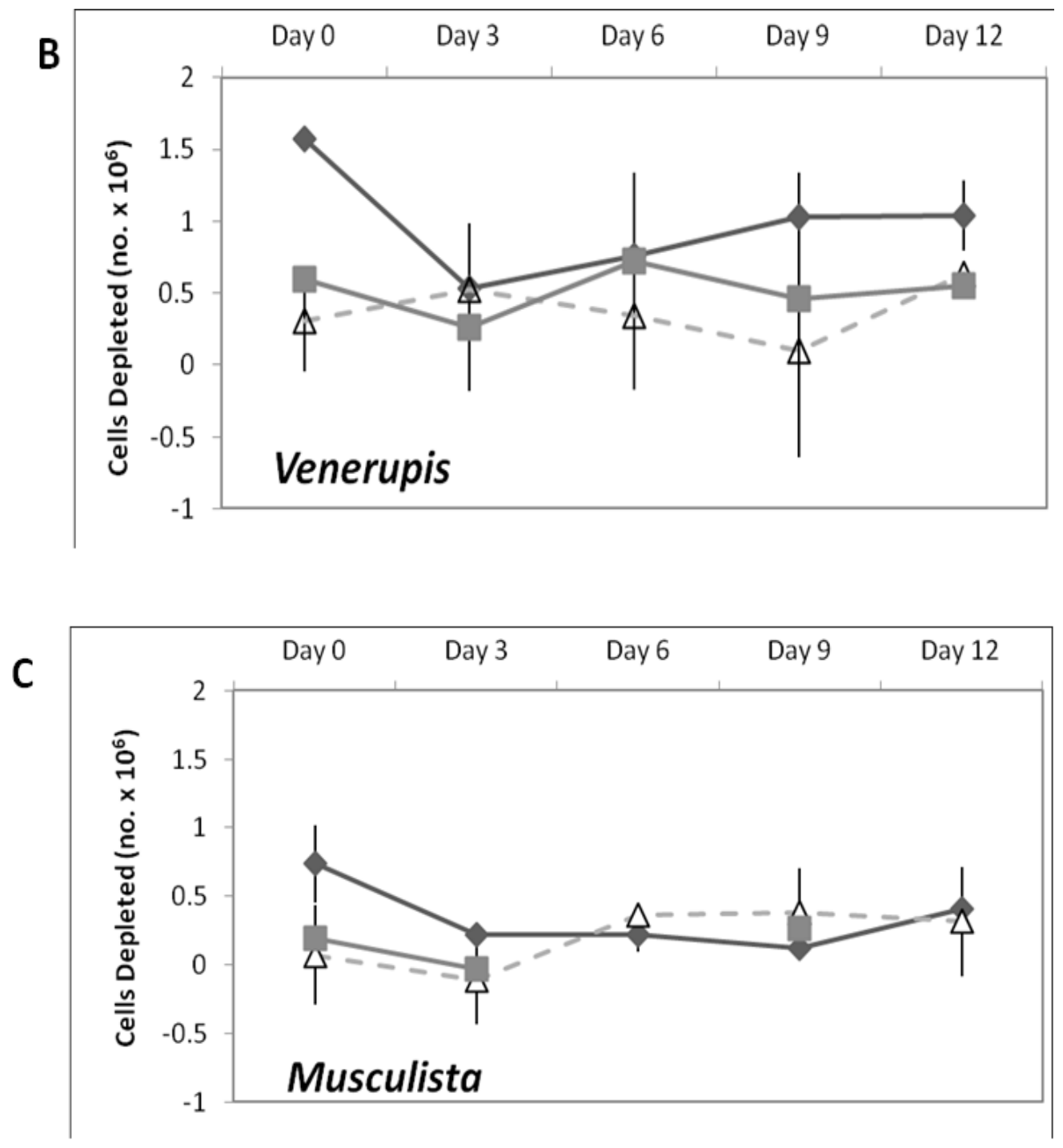
Figure 6.4. Control subtraction representation of mean algae depletion rates for 12 day experiment by concentration. Mean total algae depletion rates with control subtracted for Chione californiensis, Venerupis philippinarum, and Musculista senhousia over 12 days in Low, Medium, and High copper treatments. Algae depletion rates in control chambers were subtracted from the depletion rates measured in chambers containing bivalves. Error bars represent \pm one standard deviation.
A.) Low copper treatment
B.) Medium copper treatment
C.) High copper treatment 


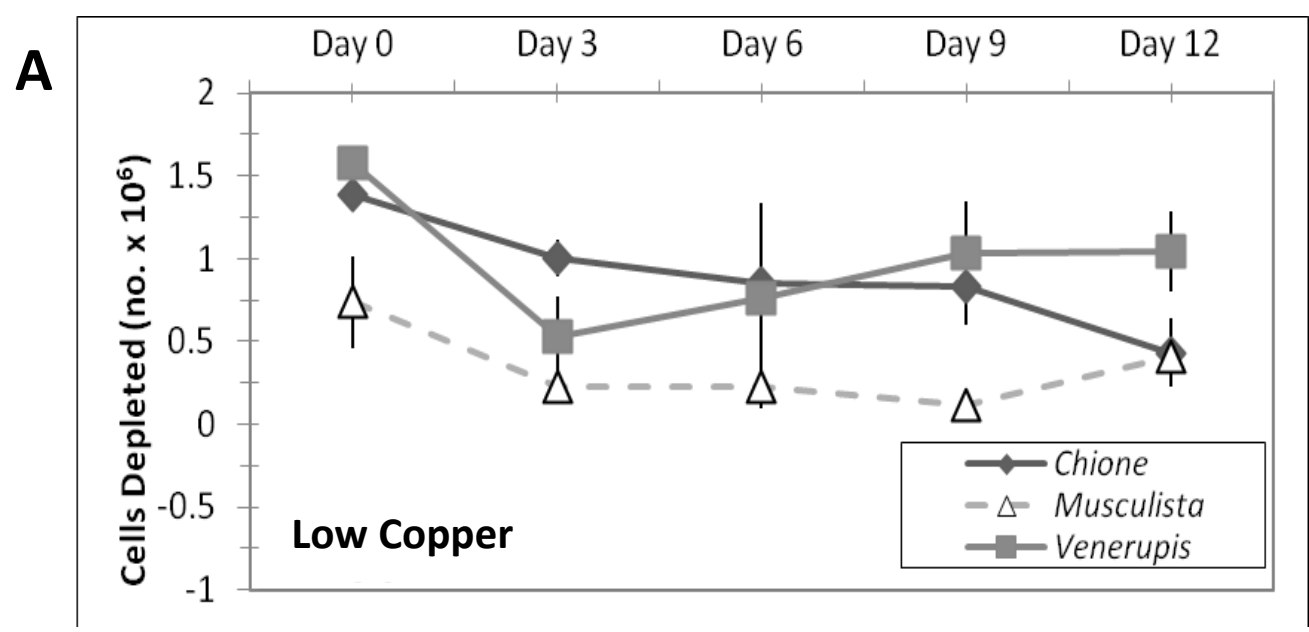

B
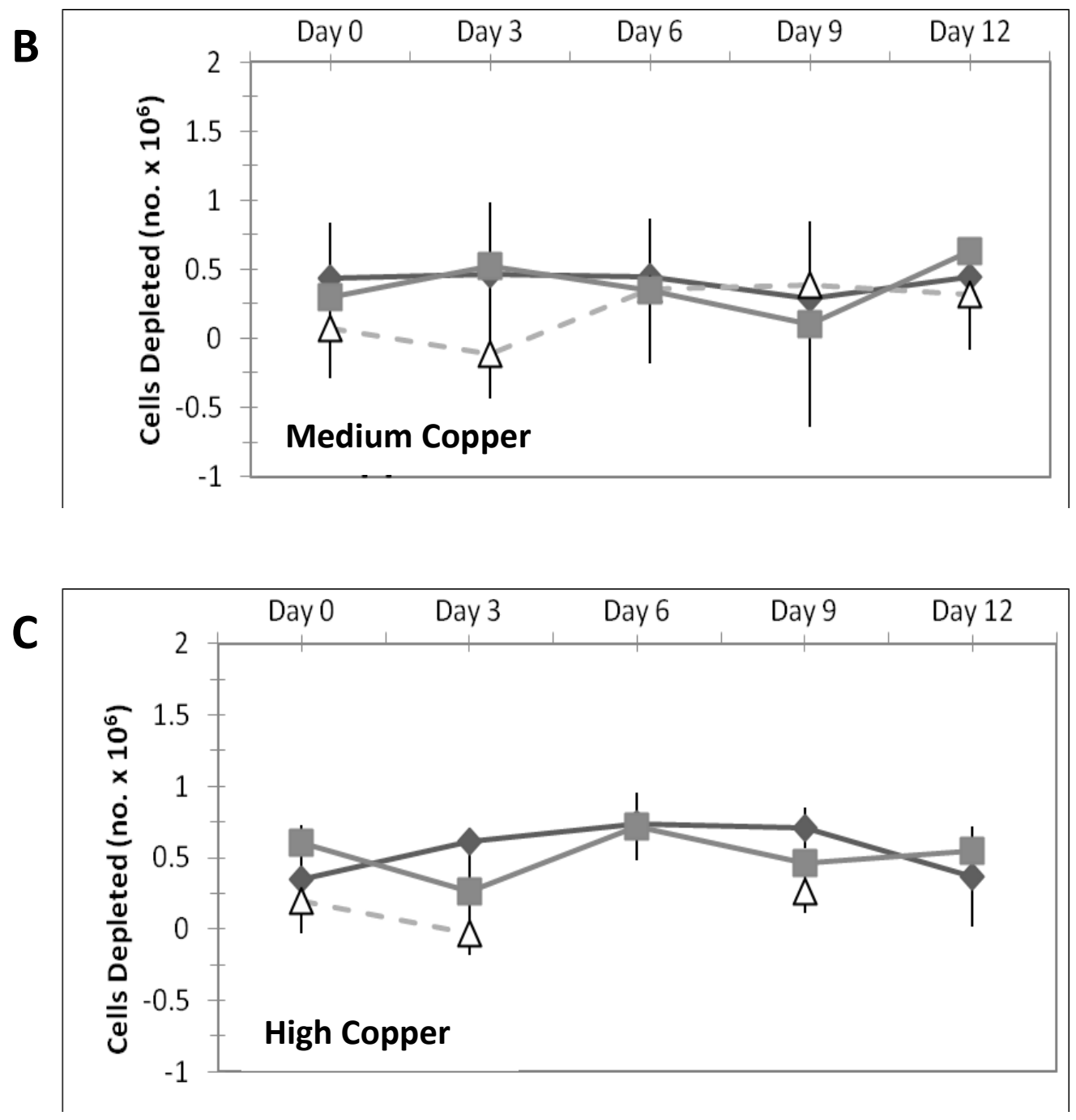
Figure 6.5. Low copper treatment subtraction representation of mean algae depletion rates for 18 day experiment by concentration. Mean total algae depletion rates with low copper treatment subtracted for Chione californiensis, Venerupis philippinarum, and Musculista senhousia over 18 days in Medium and High copper treatments. Error bars represent \pm one standard deviation.

A.) Medium copper treatment

B.) High copper treatment 


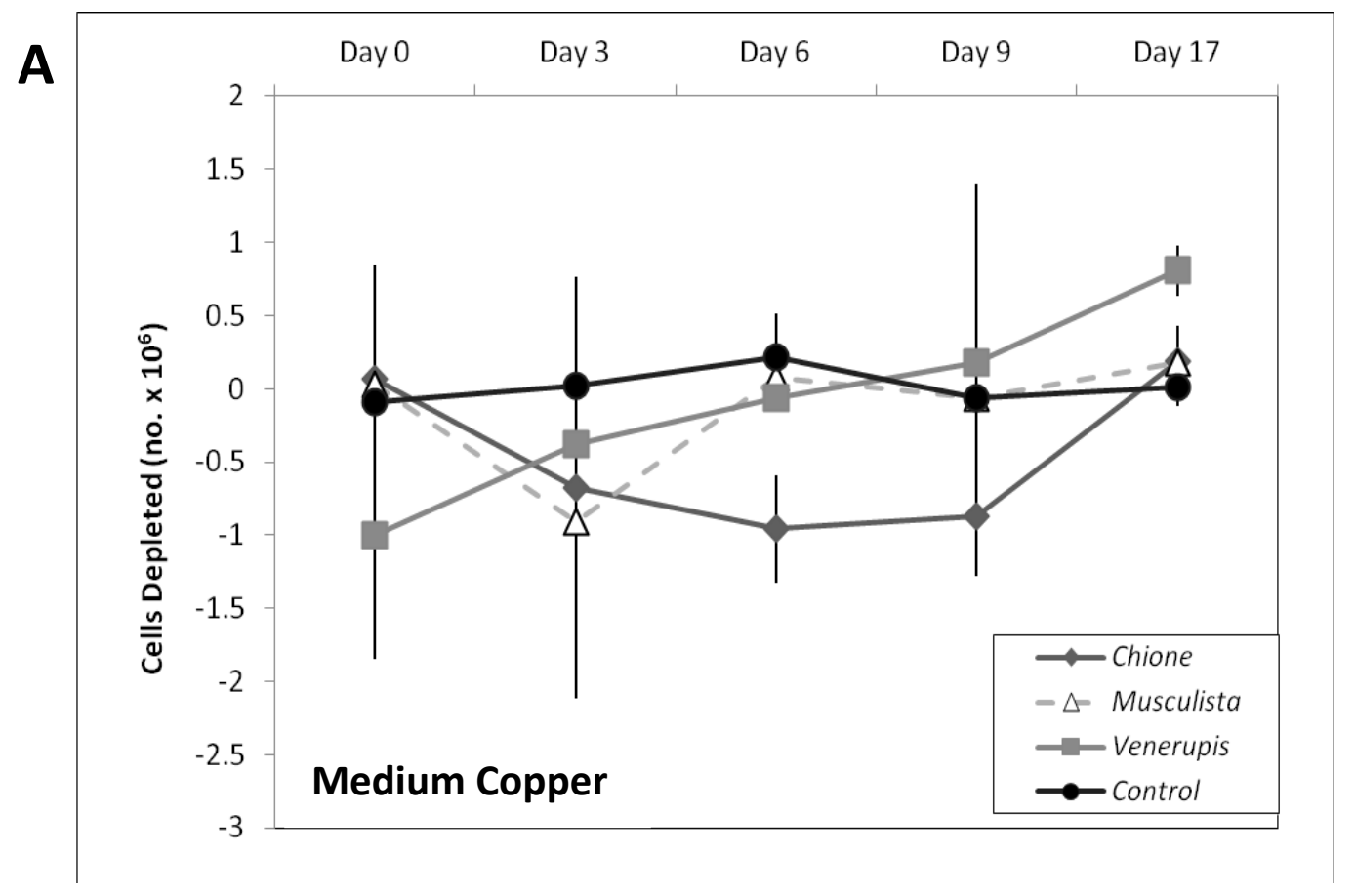

B

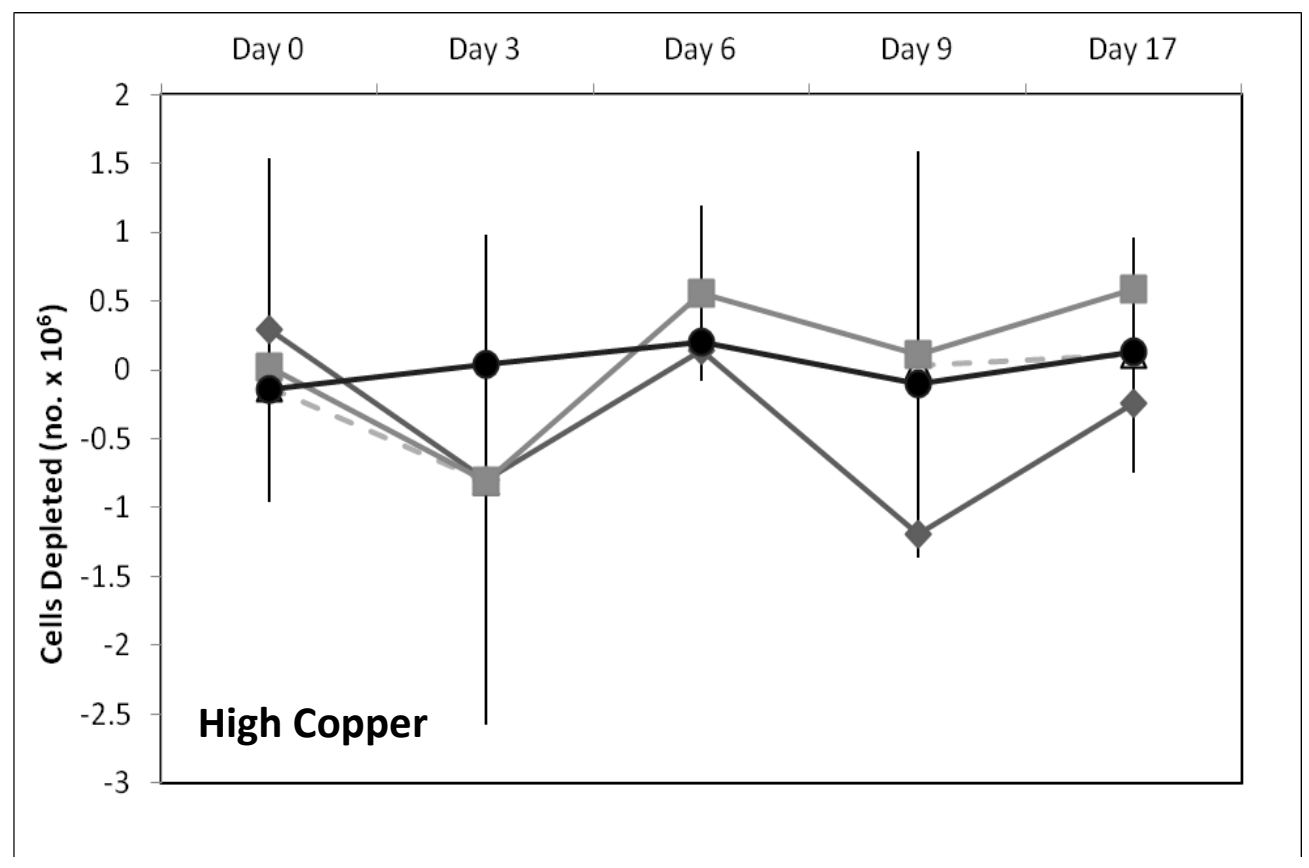


Figure 6.6. Low copper treatment subtraction representation of mean algae depletion rates for 18 day experiment by species. Mean total algae depletion rates with low copper treatment subtracted for Chione californiensis, Venerupis philippinarum, and Musculista senhousia over 18 days in Medium and High copper treatments. Error bars represent \pm one standard deviation.
A.) Chione californiensis

B.) Venerupis philippinarum

C.) Musculista senhousia 
A

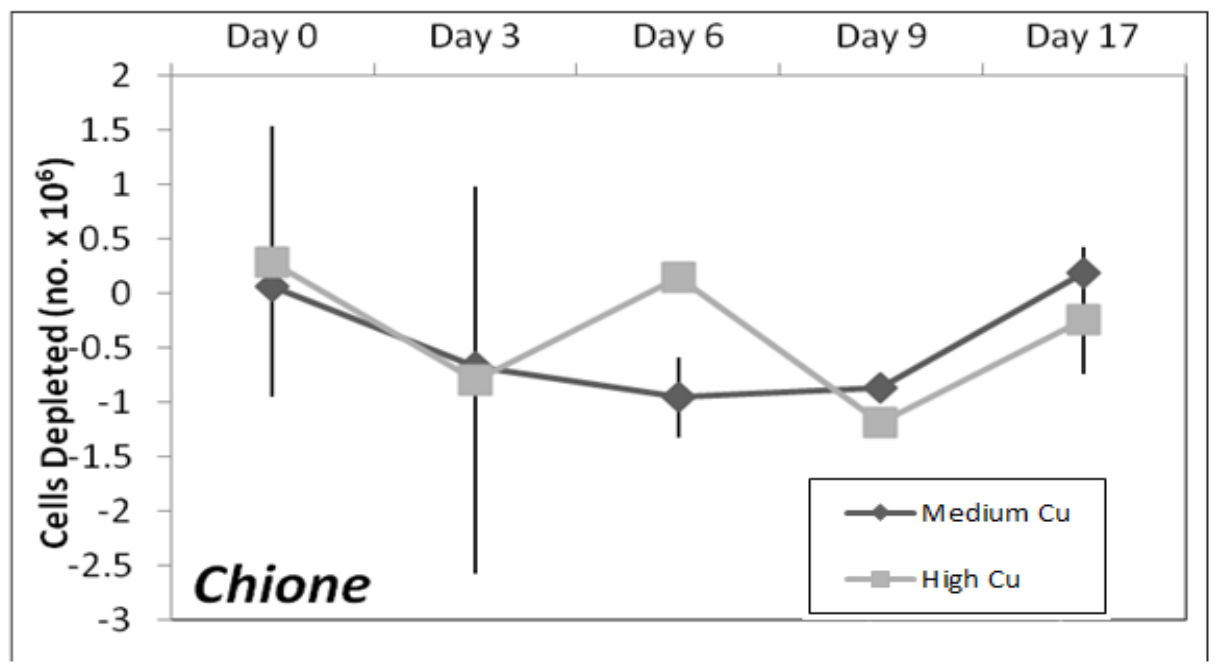

B

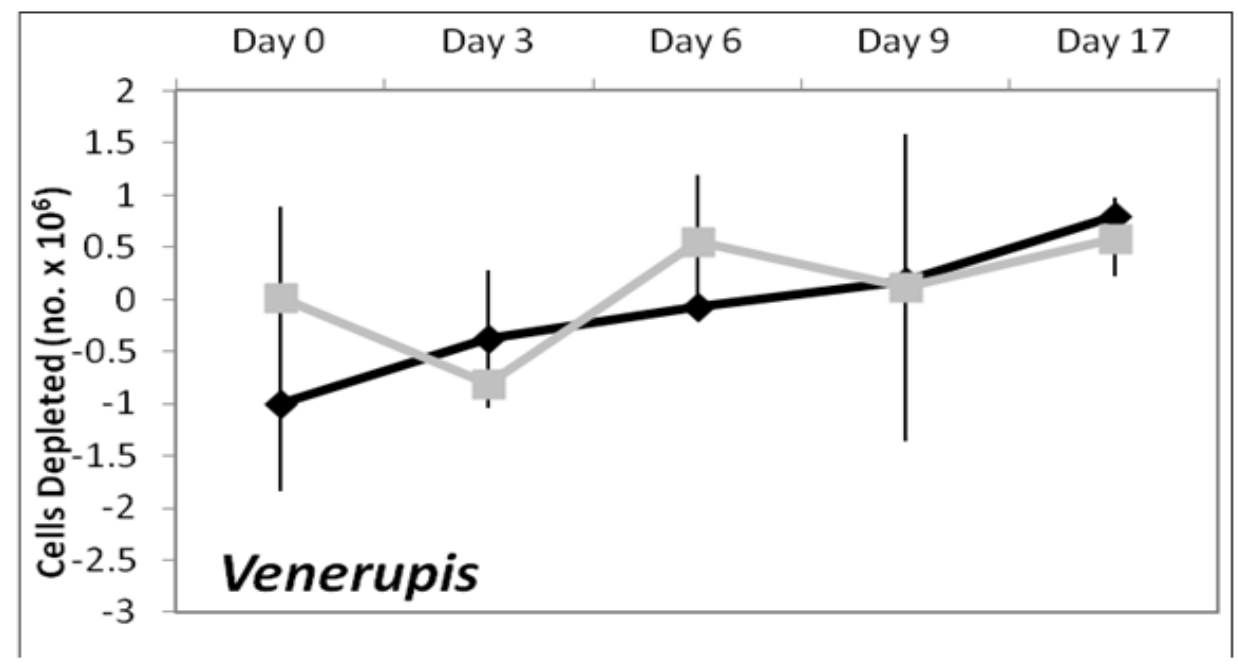

C

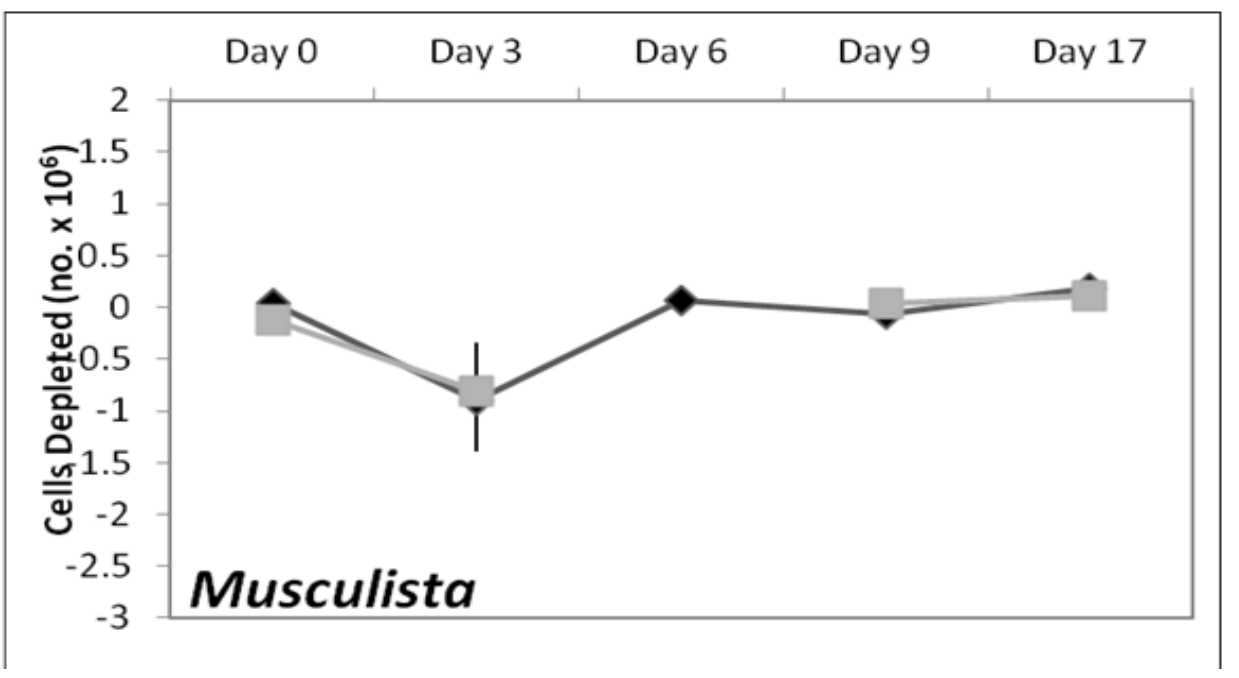


Figure 6.7. Low copper treatment subtraction representation of mean algae depletion rates for 12 day experiment by concentration. Mean total algae depletion rates with low copper treatment subtracted for Chione californiensis, Venerupis philippinarum, and Musculista senhousia over 12 days in Medium and High copper treatments. Error bars represent \pm one standard deviation

A.) Medium copper treatment

B.) High copper treatment 


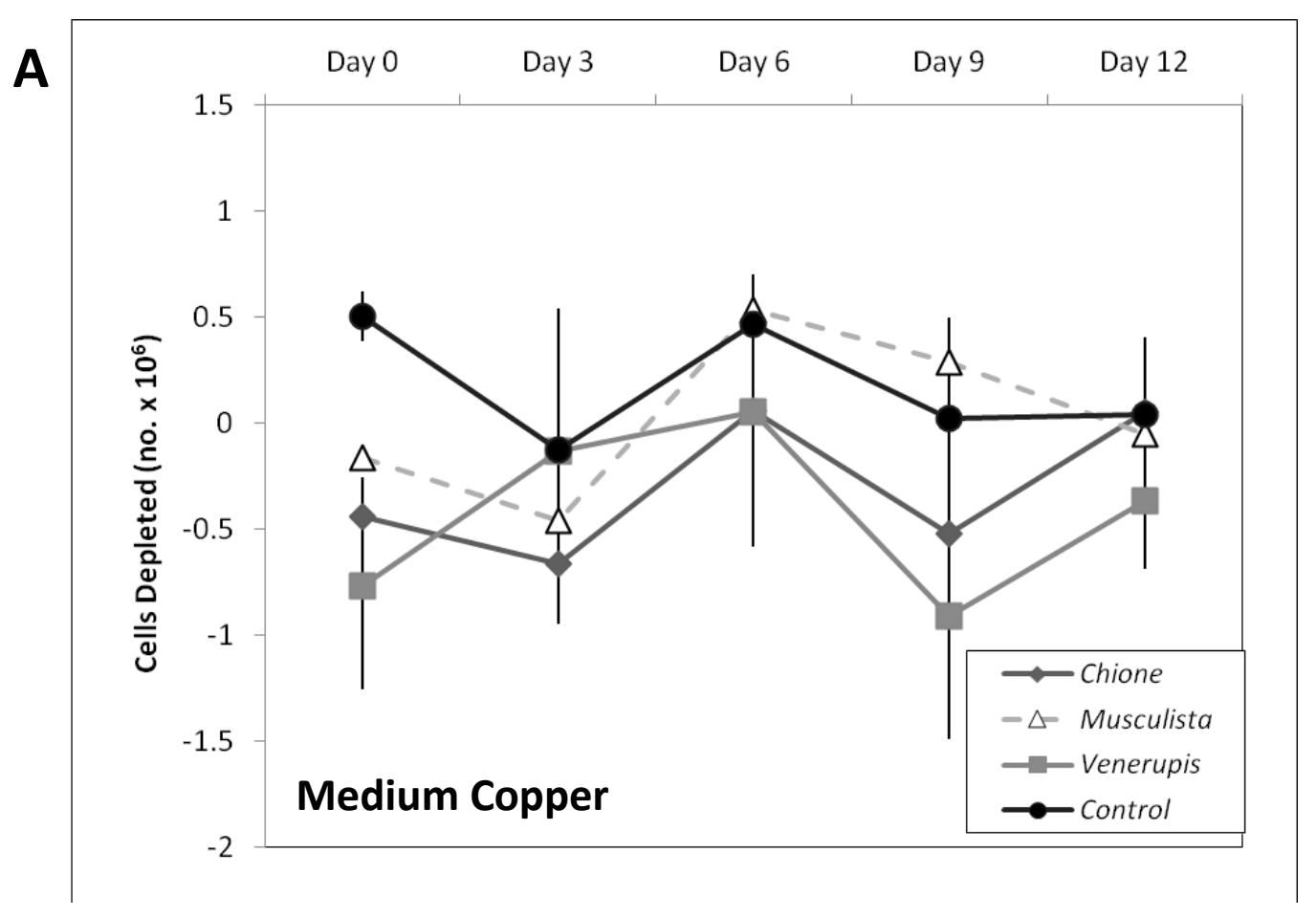

B

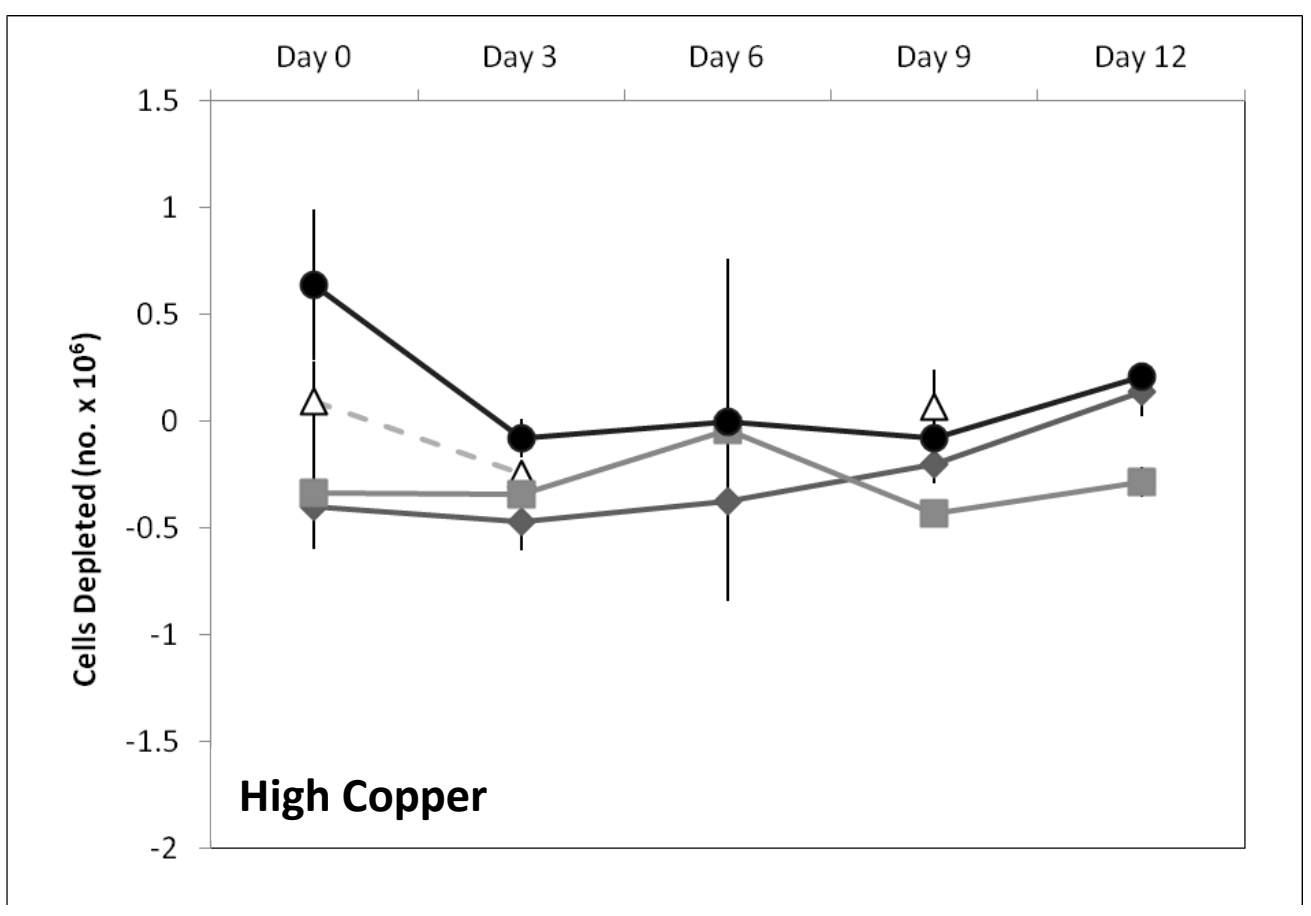


Figure 6.8. Low copper treatment subtraction representation of mean algae depletion rates for 12 day experiment by species. Mean total algae depletion rates with low copper treatment subtracted for Chione californiensis, Venerupis philippinarum, and Musculista senhousia over 12 days in Medium and High copper treatments. Error bars represent \pm one standard deviation.

A.) Chione californiensis

B.) Venerupis philippinarum

C.) Musculista senhousia 
A

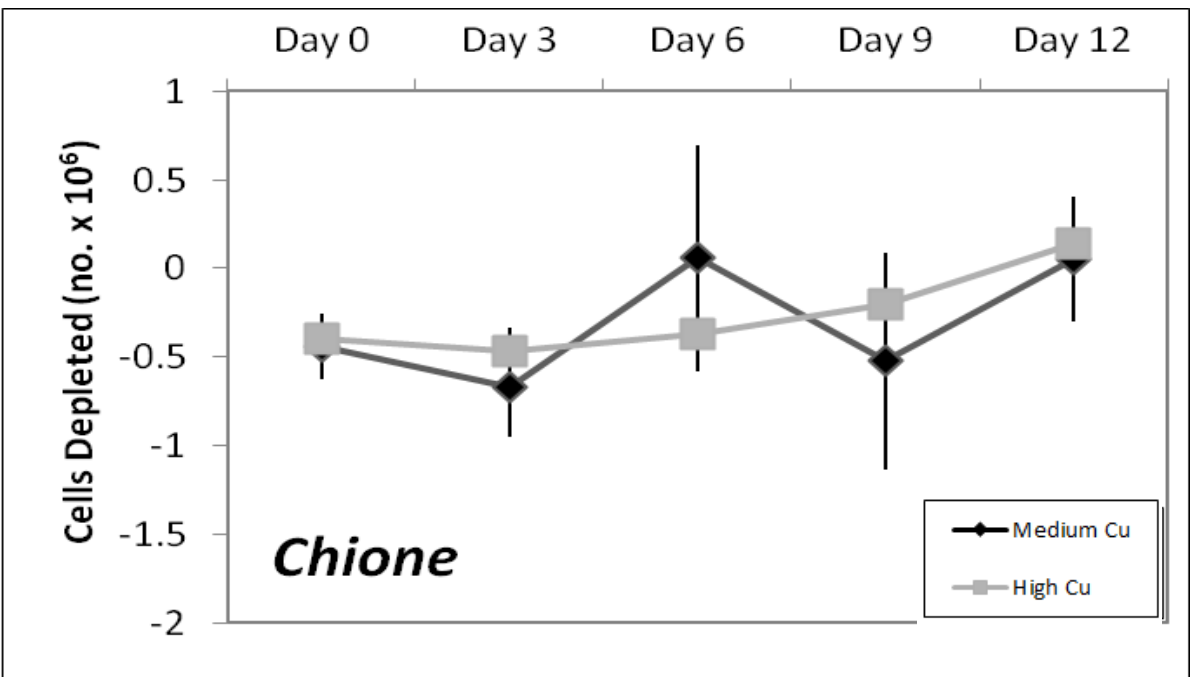

B

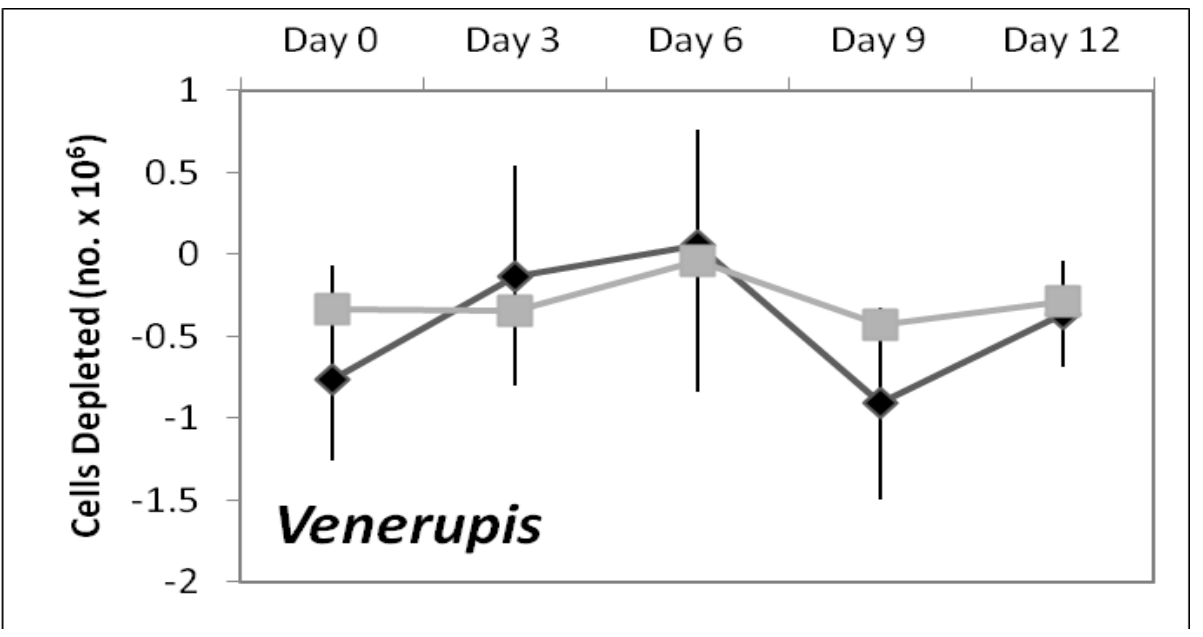

C

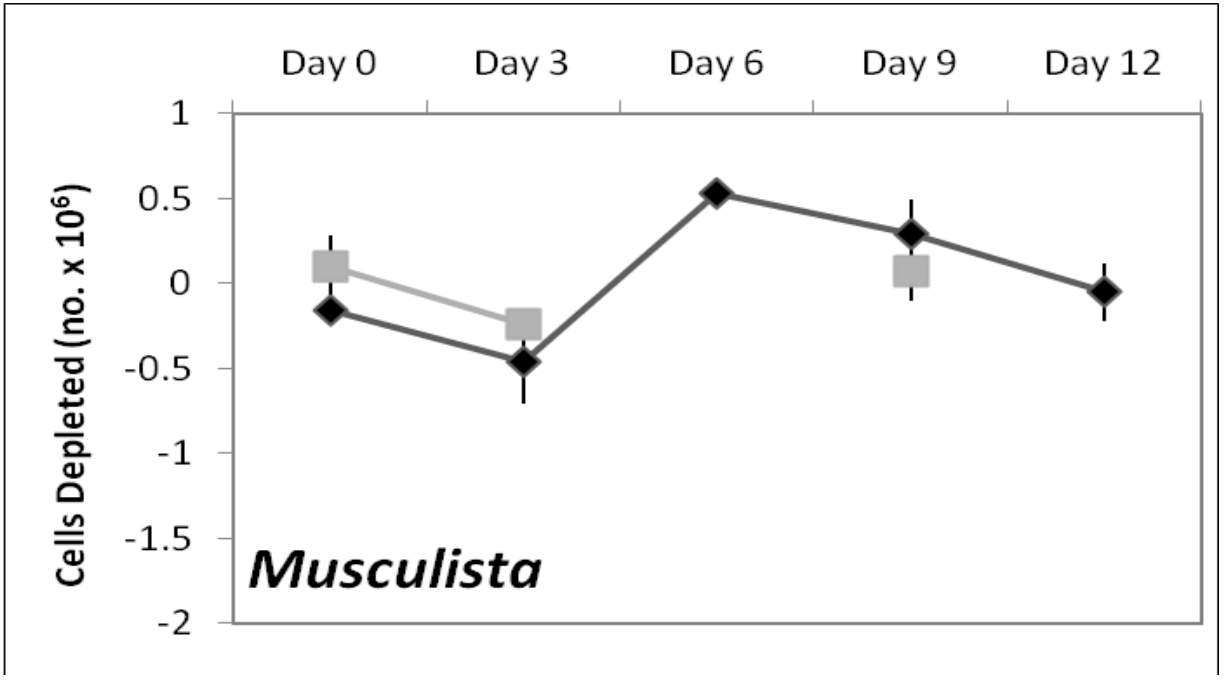




\subsection{Discussion}

The focus and inspiration for this study were derived largely from a combination of two issues that could have widespread consequences, both in the present and the future. The first issue is that of copper pollution, a problem that is particularly pertinent to San Diego and other coastal communities. Moving forward, the issue of copper in coastal ecosystems is likely only to increase in severity, as there are several sources of ongoing copper input such as runoff from roads, and more importantly, antifouling paint from boats (Lewis 1995). Copper is an important element in antifouling paints, and it stands to reason that unless there is a change in the method of antifouling, copper will continue to leach into coastal ecosystems through bays and harbors (Lewis 1995).

In a survey of Mission Bay, dissolved copper concentrations as high as $26.6 \mathrm{ppb}$ were measured (Kaufmann et al. 2004), and in 1996 copper levels in San Diego Bay were as high as $23.5 \mathrm{ppb}$, both similar to the Medium copper treatment used in this experiment (Zirino et al. 1996). In contrast, in 1998-1999, Rivera-Duarte and Zirino took water samples from different locations around the San Diego area, including sampling locations from the coastal waters near Scripps Institution of Oceanography (SIO), and in Mission Bay and San Diego Bay. Dissolved copper concentrations ranged from $0.3 \mathrm{ppb}$ at $\mathrm{SIO}$ to $9.2 \mathrm{ppb}$ at Shelter Island, with a mean concentration of $3.3 \mathrm{ppb}$ (Rivera-Duarte and Zirino 2004). Thus, this experiment can provide a relevant benchmark to indicate some of the biological effects of copper concentrations that have been measured and could be reached in the future, with the $50 \mathrm{ppb}$ concentration being used as a high-level indicator of potential future copper concentrations and of concentrations in more 
contaminated environments. In this sense, parameters for this experiment are realistic in their relation to copper levels found in coastal environments, with the Low copper treatment representing the contemporary low copper concentrations in San Diego's coastal waters and bays, the Medium copper treatment representing the highest environmental concentrations measured in the San Diego area in recent years, and the High copper treatment representing an environment with heavy copper contamination. The study was designed to take a closer look at the effects of these copper levels because of the very real potential for these species to face contamination of a similar nature, both now and in the future.

A pilot study for this experiment was used to determine the target copper exposure for the High exposure group, and indicated that $50 \mathrm{ppb}$ showed the highest mortality while still having specimens from all three species survive for sublethal factor testing (Table 8.1). Other studies have used similar concentration ranges to examine the effects of copper on bioassay organisms. Al-Subiai et al. (2011) used a range of 0 to 56 ppb in their study of copper effects on Mytilus edulis, and Vosloo et al. (2012) used 0 to $50 \mathrm{ppb}$ copper concentrations for their study of the effect of copper on Perna perna. Thus, the range of copper concentrations used in this experiment was not unusual in comparison to the ranges used in previous studies that examined the effects of copper on bivalve species.

The second aspect of this study touches on a problem that is plaguing ecosystems throughout the world: the proliferation of non-indigenous species and their spread into new territories (Piola et al. 2009). Two of the three species used in this experiment were non-indigenous, and have been shown to have a direct impact on 
native species in the areas they have invaded (Crooks 1998a). Previous studies in Mission Bay have shown a steep drop in population density of native Chione species following the proliferation of the invasive Musculista senhousia (Crooks 1998a). Knowing the negative impact of non-indigenous species on native populations already, this study examined whether elevated copper levels would alter the competition between native and non-indigenous species. Piola et al. (2008) showed the ability of some non-indigenous bryozoan species to be more resistant to copper than native species, giving them an advantage in copper contaminated environments. Crooks et al. (2011) reported that, across several different taxa exposed to copper, native species average species richness on settlement plates dropped in a statistically significant manner, exhibiting an overall $40 \%$ decline. Non-indigenous species average species richness, however, did not show a significant change. These results indicate that some non-indigenous species have a competitive advantage in surviving in copper contaminated environments, and helped inspire the non-indigenous/native species comparison in this study.

\subsubsection{Water Quality}

Measured concentrations of dissolved copper did not match the expected concentrations of 0,25 , and $50 \mathrm{ppb}$ (Figure 1.2, Figure 1.3). A similar study involving the dosing of tanks with dissolved copper produced similar variation, with the greatest variation between theoretical and measured copper occurring at higher concentrations. Al-Subiai et al. (2011) measured differences as high as $25 \%$ between expected and measured copper concentrations in the exposure solutions that they used. Their copper 
concentrations ranged from the control group of $0 \mathrm{ppb}$ to a maximum exposure of 100 ppb. They observed that the actual copper concentrations varied more as predicted values increased and suggested that this result might have been the result of copper precipitation at higher concentrations. Similarly to Al-Subiai et al. (2011), higher copper concentrations were among the most variable in this experiment as well. However, in this experiment, copper concentrations in the Low copper treatment were higher than those found by Al-Subiai et al. (2011), which may be the result of differences between the background copper concentrations in the sources of filtered seawater.

Contamination, precipitation, adsorption, natural variation and human error are possible reasons for the variation among tanks. As there were several steps in the creation and administration of the stock solution, there are several areas where the discrepancy in copper could have occurred. Slight variations in the amount of copper sulfate pentahydrate used to generate the stock solution could have had an amplified effect, as a slight variation between the generated stock solutions when dosing $20 \mathrm{~L}$ could lead to larger changes and result in the variation observed in copper levels.

With regard to the Low copper treatment, there are natural levels of copper in seawater, which often vary among locations (Lewis, 1995). Thus, natural seawater would not be expected to be entirely free of dissolved copper. Al-Subiai et al. (2011) measured background copper concentrations of filtered seawater in their experiments as approximately $1.46 \mathrm{ppb}$ but referred to the control group as "0 ppb." In comparison, the mean copper concentration of the seawater in the Low copper treatment in this study was higher, at $6.50 \pm 1.11 \mathrm{ppb}$. Similarly, even in Ventura point, the area of Mission Bay considered to have high circulation and flushing to the ocean, 
measurements of as high as $16.0 \mathrm{ppb}$ were observed (Kaufmann et al. 2004), indicating that even areas not expected to be associated with high levels of copper concentration can exhibit background copper levels. Other time points at the same location in Mission Bay yielded copper concentrations of $6.86 \mathrm{ppb}$ and $7.86 \mathrm{ppb}$ (Kaufmann et al., 2004), which were more in line with the background copper measured in this experiment.

For the Medium and High copper treatments, the measured copper level turned out to be less than expected. Studies involving copper in seawater have speculated that lower measured vs. calculated copper concentrations could be the result of copper precipitation or adsorption to surfaces (Nadella et al. 2009, Al Subiai et al. 2011). In this study, attempts were made to mitigate the precipitation of copper from the water by using acrylic tanks and plastic beakers (Nadella et al. 2009). Organic material in seawater also can result in a removal of copper from the water (Zirino et al. 1998, Strom et al. 2011), and the production of organic matter by the bivalves may have affected copper concentrations in the exposure tanks.

Variation also was observed between the two experiments. In general, the 12 day treatment groups had higher copper concentrations than their counterparts in the 18 day experiment (Figures 1.3, 1.4). For the Low and Medium copper treatment groups, this difference was not statistically significant. However, a t-test indicated that there was a statistically significant difference $(p=0.0249)$ between the copper concentrations in the High copper groups in the 18 day (mean $=40.73 \mathrm{ppb}$ ) and 12 day (mean $=47.53$ ) experiments. The ramifications of this difference will be discussed in the survivorship section. An ANOVA comparing the measured copper in the three treatment groups showed statistically significant differences, with post hoc testing 
showing significant differences between all pairs of treatment groups. Therefore the principles behind the experiment and the desire to examine differences between copper concentrations are still supported, even if the calculated and measured copper concentrations were different.

Differences between predicted and measured copper concentrations are important to note, as in others using similar methods (Anandraj et al. 2002, Vosloo et al. 2012). Anandraj et al. (2002) addressed this difference by adding copper to the experimental tanks each day of the study and reporting a range of $44-48 \mathrm{ppb}$ rather than a single concentration. Vosloo et al. (2012) had an exposure time of only 24 hours and addressed the discrepancy in calculated vs. measured copper levels by measuring both an initial copper concentration and a copper concentration at the time of water change and comparing both to the calculated copper concentrations.

\subsubsection{Copper accumulation}

Whole body soft tissue analysis is the primary measurement for copper accumulation when using tissue copper as a sublethal marker for the impact of copper on a bivalve, with a number of examples of significant differences between copper exposure groups and control groups showing that tissue copper increases with increases in copper exposure (Grout and Levings 2001, Turner et al. 2009, Al-Subiai et al. 2011, Vosloo et al. 2012). The data from this study mostly matched the results from these other studies. Perhaps the most interesting result was the significant difference between Musculista and Chione in whole body copper, which, when coupled with the higher mortality levels of Musculista, contradicts the established research of non- 
indigenous species having a competitive advantage over native species in contaminated environments (Lee and Chown 2007, Piola and Johnston 2008).

Whole body copper in the brown mussel Perna perna over a period of 24 days at measured concentrations of 44-48 ppb showed an increase over time (Anandraj et al. 2002). At day 16, the time point most comparable to the duration of this study, mean whole body copper concentration was $23 \mu \mathrm{g} \mathrm{g}^{-1}$. In this study, whole body copper concentrations in bivalves from the High copper group (measured at $44 \mathrm{ppb}$ ) in the 18 day experiment were $85.83 \mu \mathrm{g} \mathrm{g}^{-1}$ for Chione, $64.46 \mu \mathrm{g} \mathrm{g}^{-1}$ for Venerupis, and $194.87 \mu \mathrm{g} \mathrm{g}$ ${ }^{1}$ for Musculista. For the 12 day experiment, mean whole body copper concentrations were $56.93 \mu \mathrm{g} \mathrm{g}^{-1}, 58.06 \mu \mathrm{g} \mathrm{g}^{-1}$, and $202.20 \mu \mathrm{g} \mathrm{g}^{-1}$ for Chione, Venerupis, and Musculista, respectively. This result could indicate that Chione, Venerupis, and Musculista accumulate copper more quickly and to higher concentrations than Perna perna. Anandraj et al. (2002) noted that mortality at $50 \mathrm{ppb}$ was negligible for their experiment, though they did not provide mortality data. It is possible that Perna perna is better able to exclude or remove copper from its body than the species in this study, or there may be other factors, such as the fact that Perna perna is known to be an invasive species and, similarly to other non-indigenous species, may be better able to tolerate a copper-contaminated environment (Hicks et al. 2001).

A study on copper accumulation in Ruditapes philippinarum showed that copper accumulated linearly over time, but with higher accumulation rates during the first 48 hours (Sobral and Widdows 1997). If the three species in this study follow a similar pattern of increasing copper accumulation with time, then the bivalves in the 18 day experiment should have accumulated more copper than those in the 12 day 
experiment; however, this was not always the case. Copper accumulation in the two exposure groups usually showed similar concentrations at the conclusions of the two experiments. The 18 day experiment sometimes led to higher copper levels, but there were multiple instances of bivalve species accumulating more copper in 12 days than in 18 days. For example, whole body copper accumulation by Chione in the Medium copper treatment group was $52.85 \mu \mathrm{g} \mathrm{g}^{-1}$ (18 day) vs. $99.44 \mu \mathrm{g} \mathrm{g}^{-1}$ (12 day) (Appendix B, Table 2.1).

In this study, copper concentrations were examined separately in tissue from the gills and digestive tract. Across experiments, gill copper and digestive tract copper showed significant differences between exposure concentrations, as higher exposures were significantly higher than those of lower exposures. The $p$-values for the gill tissue copper were much lower than those of the digestive tract copper, which may indicate there is a stronger correlation between gill copper and exposure concentration.

Gill copper is accumulated through direct contact with water that passes over the gills during respiration (Gregory et al. 2002), whereas copper accumulation in the digestive tract is more likely to be affected by copper concentrations in the bivalve's food source and processes affecting retention of copper (Strom et al. 2011). Exchange surfaces such as gill tissue have been shown to suffer damage from exposure to dissolved copper and other harmful metals (Gregory et al. 2002, Anandraj et al. 2002, Al-Subiai et al. 2011).

Examination of organ tissue is not common in the published literature; most studies report whole body soft tissue analysis (e.g., Vosloo et al. 2012). However, AlSubiai et al. (2011) examined gill tissue from copper-exposed Mytilus edulis and 
observed a significant difference in copper accumulation between controls and exposure levels up to $56 \mathrm{ppb}$ after a 5 day exposure period. Examination of the gill structure showed deterioration that correlated with levels of copper exposure. However, Turner et al. (2009) examined copper concentrations in the gills and digestive tract of Mytilus edulis after exposure for 16 hours to copper-concentrated paint flakes and found that, while both were statistically significantly higher than in the control, the gills were much lower in copper concentration (mean: $8 \mu \mathrm{g} \mathrm{g}^{-1}$ ) than the digestive tract (mean: $62 \mu \mathrm{g} \mathrm{g}^{-1}$ ).

Turner et al. (2009) found that copper concentrations were highest in the digestive tract and lowest in the gills of Mytilus edulis over the course of 16 hour exposures. In this study, copper accumulation was shown to be similar or slightly higher in the digestive tract in comparison to the gills, but didn't exhibit a pattern of increasing accumulation with increasing exposure levels, as was the case for the whole body and gill tissues (Figures 2.1, 2.2). It should be noted that Al-Subiai et al. (2011) also observed an increase in copper concentration in the digestive tract, but concentrations in the gills were significantly higher at the two highest copper concentrations ( 32 and $56 \mu \mathrm{g} \mathrm{L}^{-1}$ ), but not at lower concentrations. It is possible that some bivalves are able to eliminate copper from the digestive tract through defecation over time, while copper that accumulates in the gills is bound to the tissue and unable to be expelled. Turner et al. (2009) theorized that the digestive tract was able to return copper to the water column through remobilization during the process of digestion, with some being removed via feces. If these processes take place, it could explain why there was a consistent accumulation trend in the gill tissue but not in the digestive tract tissue, particularly in the higher concentration exposures and in the 18 day experiment. 


\subsubsection{Survivorship}

Survivorship results were analyzed by copper exposure level and by species. Studies have clearly indicated that, as copper exposure levels increased, survivorship of the test subjects typically decreased (Al-Subiai et al. 2011, Vosloo et al. 2012). The results from this study were no different. Across all species, there was a significant negative correlation between copper concentration and the survivorship of bivalves (Appendix B, Table 3.1, Figure 3.1).

There was an observable difference between survival rates in the 18 and 12 day experiments. Overall, specimens in the 18 day experiment had higher survival rates than those in the 12 day experiment, in spite of a longer exposure period. One possible explanation for this result comes from measured copper concentrations, which were lower, significantly so for the High copper treatment groups, in the 18 day experiment than the 12 day experiment (Figure 1.4A). With this difference in mind, the significant difference between the initial copper concentrations for the High copper treatment groups in the 18 day and 12 day experiments should be addressed. In general, in the High copper groups, there was a higher survival percentage among all species in the 18 day study. Mean survivorship rates in the 18 day experiment for Chione, Venerupis and Musculista were 73.3\%, 26.7\%, and 26.7\%, respectively, generally higher than in the 12 day experiment (46.7\%, 20.0\% and $67.0 \%$, respectively). However, these differences were not statistically significantly different for any of the three species for either experiment ( $p=0.790$ and 0.597 , respectively).

The survivorship results show that mortality increased substantially between the Medium and High copper treatments, with Chione showing a decrease in 
survivorship of $13.3 \%$ for the 18 day experiment and $53.3 \%$ for the 12 day experiment (Figure 3.1, Table 3.1). Similar decreases were observed for Venerupis (60\% decrease in survivorship for both experiments) and Musculista (a decrease of $66.7 \%$ for the 18 day experiment and 53.3\% for the 12 day experiment) (Figure 3.1, Table 3.1). This result suggests the existence of a critical concentration beyond which mortality levels would be expected to increase sharply. This "threshold" concentration is likely to vary among bivalve species, as has been reported in past studies (Sunila 1981, Sunila and Lindstrom 1985, Widdows and Johnson 1988, Grout and Levings 2001). It is reasonable to conclude that such thresholds exist for the three species studied here, and the survivorship trends suggest that threshold concentrations are between the exposure levels for the Medium and High copper treatment groups (23.1-44.1 ppb).

Though in a different context, support for the notion of a threshold was observed by Grout and Levings (2001). In their examination of the effects of copper on survival of $M$. edulis, they observed that survival decreased at a linear rate between $0 \mu \mathrm{g}$ $\mathrm{g}^{-1}$ and about $35 \mu \mathrm{g} \mathrm{g}{ }^{-1} \mathrm{dry}$ weight tissue copper, then dropped severely from $\sim 80 \%$ to $\sim 20 \%$ at $40 \mu \mathrm{g} \mathrm{g}^{-1}$, following what they referred to as a threshold model. The idea of a critical threshold was also observed by Widdows and Johnson (1988), who reported a mortality threshold at $59 \mu \mathrm{g} \mathrm{g}^{-1}$ for M. edulis. A threshold was also observed in survival of M. edulis in copper contaminated water by Sunila (1981), with a sharp drop in survival at approximately $60 \mathrm{ppb}$. The mechanism responsible for this mortality threshold may be related to tissue accumulation of copper (e.g., Widdows and Johnson 1988).

Survivorship data from this study were examined for potential differences between native and non-indigenous species. Although the native Chione species 
appeared to have a much higher survival rate than the non-indigenous Venerupis and Musculista, particularly in the High copper treatment groups, this observation was not supported statistically in a Kruskal-Wallis test for each experiment $(p=0.790$ for the 18 day experiment, and $p=0.597$ for the 12 day experiment).

An examination of the relationship between the species within the individual exposure groups revealed some statistical significance. When examining only the High copper treatment groups for the 18 day experiment, there was a statistically significant difference shown in a Kruskal-Wallis test between Chione and the other two species $(p=$ 0.009). In order to further examine potential relationships between survivorship and species and to increase sample size, the High copper treatment groups from the 18 day and 12 day experiments were combined and differences in survivorship among species were tested. Survivorship data from the 18 day experiment were evaluated at the 12 day mark to provide a comparable time point with the 12 day experiment. There was a statistically significant difference between the species in the High copper treatment $(p=$ 0.0224), with Chione displaying significantly higher survivorship than Venerupis or Musculista. This result suggests that the native species in this study was more tolerant of elevated copper concentrations than the two non-indigenous species. A similar examination of Medium copper values using a one-way ANOVA showed there was no significant difference between species for either experiment $(p=0.949$ and 0.079 , respectively).

In this study, the native species Chione californiensis exhibited higher survivorship in the High copper treatment than the non-indigenous species tested in this study. While the differences weren't evident in the Low and Medium copper treatment 
groups, in the High copper treatment group, Chione had a much higher survivorship than Musculista or Venerupis, (60\% vs. $20 \%$ and $30 \%$, respectively). These findings are contrary to expectations based on published data (Piola and Johnston 2008, 2009, Piola et al. 2009, Crooks et al. 2011). In direct comparisons between control groups and groups exposed to copper from antifouling paint, the presence of copper may give nonindigenous species a competitive advantage over native species (Piola and Johnston 2008, 2009, Piola et al. 2009). Crooks et al. (2011) showed that aquatic copper pollution tended to increase the success of invasive species. In general, it appears that the presence of copper may benefit non-indigenous species and harm populations of native species, as the introduction of copper reduced the total native species richness on settlement plates by more than $40 \%$ (Crooks et al. 2011). One study on invasive bryozoan species indicated that copper tolerance may be heritable, suggesting that, at least in some instances, invasive species have adapted to better tolerate copper (McKenzie et al. 2011). It should not be overlooked that Chione californiensis was the most common native bivalve species encountered when sampling in Mission Bay, and that Mission Bay has been shown to have, at least in some areas, fairly high levels of copper contamination (Kaufmann et al. 2004). While the ability to survive under coppercontaminated conditions may not be the only reason that Chione californiensis was collected in high numbers in Mission Bay, the presence of this species in relatively large numbers in a contaminated environment suggests some level of copper tolerance or resistance.

A future study would have to go further, and look at the effects of copper on juveniles and larvae to see if Chione truly would have a competitive advantage in a copper-contaminated environment. Another future study could examine the factors 
driving the relatively high abundance of Chione californiensis in Mission Bay. It may be that its abundance is a result of being more tolerant of copper and other anthropogenic pollutants than other native species, but this would need to be tested against other native species and survivorship variables. This situation may also be true to some extent for Musculista and Venerupis, which were the non-indigenous species most frequently encountered when collecting bivalves for this study. Expanding the scope of this study to examine a wider variety of native and nonindigenous bivalve species would also better characterize the relationship between copper toxicity and resistances among native and nonindigenous species of bivalves.

An interesting point to consider is the effect of body size on sensitivity to environmental contaminants such as copper. In one study, body size was shown to have a positive correlation with copper accumulation; however the authors indicate that the increase of copper accumulation was likely due in part to the age of the animal, as larger specimens tend to be older and are potentially exposed to environmental contamination longer than smaller, younger specimens (Ahn et al. 2001). Thus, it seems possible that body size is an important factor that contributes to the difference in survivorship between Chione and Musculista, which have a large size disparity. Venerupis is a much better comparison for Chione in terms of body both size and perhaps physiology, as they are both clams, whereas Musculista is a mussel and exhibits both faster growth rates and different behavior, including living in groups and binding to surfaces using byssal threads (Crooks 1998a). There was an ANOVA of the 18 day and 12 day data combined that indicated a statistically significant difference in species survivorship $(p=0.009)$ and a Tukey's post hoc test showing a statistically significant difference in survivorship between Chione and Venerupis $(p=0.052)$ In this case, the 
basis for Chione being better able to survive may not have been linked to body size. Venerupis tends to be larger than Chione. In this study, across experiments the mean shell width of Chione was $33.52 \mathrm{~mm}$, while the mean shell width of collected Venerupis specimens was $38.92 \mathrm{~mm}$. As a result, it is unlikely that body size was the main factor for Chione having a higher survival percentage than the other two species. In addition, Venerupis and Musculista, in spite of their larger size disparity, displayed similar survival percentages throughout the study.

Survivorship was also examined over time to evaluate possible causes of mortality in relation to particular events during each experiment.

The Low copper treatment tanks never had mortalities higher than one per species per day, and those mortalities were not clustered in time or in specific tanks. Also, the mortalities occurred more than halfway through the experiment and several days after the water change, indicating that shock from either beginning the experiment or the water change was not a likely cause of mortality.

For the Medium copper treatment tanks in both experiments, there were mortalities either on the day of the water change or within one day after water change in 5 of the 6 tanks. In each of these tanks, one or two individuals died, never more than one per species (Figures 3.8, 3.9). Assuming that copper levels in each tank had decreased over time, the water change, which introduced new copper into the system, presumably increased copper concentrations, perhaps overburdening some of the bivalves.

The effects of the water change were both more severe and less apparent for the High copper treatment tanks, as the higher concentration of copper led to fairly 
consistent mortality prior to the water change. Unlike the mortality data from the Low and Medium copper treatment groups, the High copper mortality data weren't consistent across experiments. In the 12 day exposure, there was relatively high mortality prior to the water change and no noticeable drop in survival immediately afterward (Figures 3.8, 3.9).

In the 18 day experiment, there was little mortality prior to the water change and a decrease in survival across all species immediately after the water change (Figure 3.8). This sudden drop-off suggests that the water change may have affected bivalve mortality. Among species, Musculista died in larger numbers and had more instances of multiple deaths within the same day than the other two species. It is possible that the individual Musculista used in this experiment were particularly sensitive to copper. Also, since the Musculista were placed in a hydroponics basket that floated and physically isolated them from the other species, they may have been exposed to higher localized copper concentrations compared to individuals of the other two species, located elsewhere in the tank.

Another possible explanation for higher mortality after the water change in the 18 day experiment is based on the copper concentrations measured at the time of the water change. On average, the copper concentration of the water just before the water change was $29.8 \mathrm{ppb}$ for the High copper treatment tanks, an average difference of 10.9 $\mathrm{ppb}$ from the initial copper concentration with a standard deviation of 0.79 . By comparison, for the 12 day experiment the average difference was only $4.97 \mathrm{ppb}$, although there was more variation between tanks, as the standard deviation was 7.65. This was due to one of the tanks having a higher copper concentration at the time of the 
water change than the initial copper concentration. Thus, the water change introduced a greater increase in copper exposure for bivalves in the 18 day experiment compared to the 12 day experiment. This increase may have contributed to a shock from which some individuals were unable to recover.

Close examination of the temporal trends in mortality for each tank also suggests that copper stress was the main source of mortality, and that fouling of the water by deceased bivalves was not a factor in the survivorship results (Figures 3.8, 3.9). Both the 18 day and 12 day experiments exhibited a statistically significant relationship between copper concentration and mortality in a Kruskal-Wallis test $(p=0.0212$ and 0.0262 , respectively). Dead bivalves were removed on a daily basis, which should have limited the potential for water fouling from the decay of dead tissue. The clusters of mortalities in the High copper treatment tanks could suggest decay as an important factor, but the lack of similar clusters of deaths in the lower concentration tanks immediately after an organism death seems to contradict that hypothesis. Even if putrefaction did play a role in the survival rates of bivalves, the fact that survival rates were lower in the higher copper concentrations support a correlation between copper concentration and survival rate.

The relationship between survivorship and mean tissue copper accumulation in each species also was informative. Copper accumulation may play a large role in mortality, with sharp decreases in survivorship as tissue copper increased in Mytilus edulis (Grout and Levings 2001). Grout and Levings (2001) reported a nonlinear negative relationship between tissue copper accumulation and survival, similar to those observed in the comparisons of whole body and gill copper to survival in this study. 
Examination of both the copper accumulation in the whole body tissue and the gills produced correlations between survivorship and accumulation that were not statistically significant, though $p$ values close to 0.05 were produced for whole body accumulation in Chione and Venerupis and for gill copper in Musculista in the 18 day experiment. Further statistical testing revealed a significant relationship in the 18 day experiment between log-transformed gill copper accumulation and survivorship in Chione, but this was not mirrored in the 12 day experiment. This result could indicate that the presence of copper in the gills may contribute to mortality of Chione in copper contaminated environments in a nonlinear fashion. Al-Subiai et al. (2011) found that copper exposure in Mytilus edulis produced hypoplasia, the loss of cilia in the gills, at all elevated copper levels. This was especially apparent at the highest exposure of $56 \mu \mathrm{g} \mathrm{L}^{-1}$, and was accompanied by a sharp decrease in particle clearance rates compared to a control.

The 18 day experiment exhibited the strongest trends in tissue copper accumulation, but statistical significance was not consistent across species or experiments. The main reason that these trends were not observed in the 12 day experiment may have roots in the differences between copper levels at the time of water change. For the 12 day experiment, whole body copper accumulation for the Medium copper treatment Chione that survived after 12 days was high compared to Chione that survived the 18 day experiment, while copper accumulation for the High copper treatment Chione was relatively low (Appendix B, Table 2.1). An examination of dissolved copper concentrations at the time of water change showed that the differences before and after the water change were higher in the Medium copper treatment vs. the High copper treatment tanks for the 12 day experiment, and highest 
in the High copper treatment tanks in the 18 day experiment (Figures 1.2, 1.3, 1.4). This result, coupled with the fact that Venerupis and Musculista both had elevated copper accumulation in the Medium copper treatment compared to the same treatment in the 18 day experiment, may have led to more variation in copper accumulation data at this concentration. In spite of this, the mortality rates in the 12 day experiment were higher for the High copper treatment groups.

As a whole, the data set at the end of the 12 day experiment was smaller due to the higher mortality observed in the Medium and High copper treatment groups. Most other studies do not look at individual tissue type. Most studies, such as Vosloo et al. (2012) have reported cumulative body copper, similar to whole body copper in this experiment. Other published studies did not typically include multiple trials in different time frames, and thus did not include comparisons between experiments.

In the 18 day experiment, all three species showed positive correlations between copper exposure and copper accumulation in both whole body and gill tissue. While there may have been additional copper accumulation in the 18 day experiment as a result of being longer than the 12 day experiment, this accumulation is not clearly seen due to the high mortality rate in the High copper treatment group in the 12 day experiment. The lower number of surviving specimens in the 12 day experiment at the High copper exposures confounded comparisons to the 18 day experiments in regards to copper accumulation, as the pool of surviving specimens for the 12 day experiment was smaller and less statistically significant than that of the 18 day experiment due to a lower $n$ and higher standard deviation (Figure 3.1). 


\subsubsection{Growth}

Most bivalves by their very nature grow at slow rates. Even the fastest of the bivalves tested, Musculista senhousia, exhibited growth in hundredths of $\mathrm{mm}$ of shell length over the course of 12-18 days. During the 12 day study, Musculista grew significantly faster than Chione or Venerupis (Figure 4.1). These results are consistent with previous research on these species, as Musculista has been shown to grow relatively quickly, compared to Chione and Venerupis (Peterson 1982, Crooks 2001).

Across species, there was no significant difference in growth percentage among copper concentrations. Based on published literature, it seems likely that the duration of the experiment was not long enough to observe meaningful growth in these species. Similar experiments with different species generally occurred over longer time periods, e.g., Grout and Levings (2001) measured the effects of copper exposure on Mytilus edulis growth over 41 days. Growth of Chione undatella measured between different seasons showed increments between -0.12 and $1.05 \mathrm{~mm}$, with a mean of $0.22 \mathrm{~mm}$ (Peterson 1982). Peterson explained the negative growth as resulting from the occasional loss of the "fragile outer margin" of the shell during the measurement process. Normalized for duration, the growth rates for Chione californiensis in this study were fairly similar to those reported by Peterson (1982) for C. undatella, with an average daily growth of 0.0038 (this study) vs. $0.0036 \mathrm{~mm}$ (Peterson).

Growth has been used as a metric to examine sublethal effects of copper on bivalves. Copper exposure has been shown to stunt growth in comparison to controls. Sunila and Lindstrom (1985) reported that exposure to copper concentrations of $0.1,0.2$ and $0.4 \mathrm{mg} \mathrm{L}^{-1}$ led to stunted growth and an increase in shell deformities in the mussel, 
Mytilus edulis. In a field study (Grout and Levings 2001), growth in juvenile Mytilus

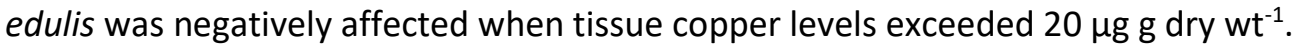
Growth rates were as high as $0.36 \mathrm{~mm}^{\text {week }}{ }^{-1}$ in low copper areas, while rates in high copper areas ranged between 0.04 and $-0.03 \mathrm{~mm}_{\text {week }}{ }^{-1}$. Grout and Levings did not propose a mechanism underlying the observations of negative growth, other than to write that mussels in the high copper areas grew poorly and were in worse condition at the end of the experiment compared to the beginning. Overall, this pattern is similar to the relationship between tissue copper concentration and growth in this study. For the most part, individual species did not show any statistically significant relationships between growth and copper accumulation in different tissues. Daily growth rates showed no significant differences between species or copper concentrations, although Musculista exhibited the highest growth rate across all copper concentrations and had a significantly higher growth percentage compared to the two larger species.

\subsubsection{Mucus production}

Mucus production is a mechanism that some bivalve species use to remove toxins or other harmful chemicals from their bodies. Sze and Lee (1995) examined the effects of copper on two bivalve species and reported that copper exposure increased mucus production. In Perna viridis and Septifer virgatus, mucus production rates increased by a factor of 2.65 and 1.85 respectively, after a three month exposure to copper at $50 \mathrm{ppb}$ (Sze and Lee 1995). Thus, the expectation in this study was that increasing copper concentrations would lead to an increase in the amount of mucus produced by the three bivalve species being examined. 
Previous studies indicated that invasive species may be more resistant to the effects of copper than native species (Piola and Johnston 2008, 2009, Piola et al. 2009). Because mucus has been shown to be a mechanism for metal removal (Sze and Lee 1995), it was expected that the non-indigenous species would produce more mucus in response to elevated copper concentrations compared to the native species.

Results from the 18 day and 12 day experiments were distinctly different, with the 18 day experiment showing the greatest mucus production by bivalves in the High copper treatment, while the 12 day experiment showed a decrease in mucus production by Chione and Musculista with increasing copper concentration. The fact that the High copper treatments had the highest mucus production and mucus/dry mass ratios agrees with previous research on mucus production in relation to copper exposure (Sze and Lee, 1995, Sze and Lee 2000). The fact that the lowest mucus production was measured in the Medium copper treatments was perplexing, as there is no obvious physiological rationale for mucus production to be higher in the Low copper treatment vs. the Medium copper treatment, though both were significantly lower than mucus production in the High copper treatment.

Mucus secretion in bivalves has been studied in relation to exposure to copper and other heavy metals; such exposure has repeatedly been shown to trigger mucus production in many species (Scott and Major 1972, D'Silva and Kureishy 1978, Sze and Lee 1995, Vosloo et al. 2012, Pillay 2013). Vosloo et al. (2012) conducted copper exposures similar to this experiment, and their data indicated that copper exposure of the brown mussel Perna perna at 25 and 50 ppb over a period of 24 hours correlated with higher levels of mucus secretion than a $0 \mathrm{ppb}$ control. Another study on Perna 
showed a similar effect over a 24 hour copper exposure period for concentrations ranging from 0-50 ppb, with significantly higher mucus production rates at higher copper concentrations (Pillay 2013). Separate experiments with the mussels Perna viridis and Septifer virgatus showed marked increases in mucus production following a 3 month exposure period at $50 \mathrm{ppb}$ (Sze and Lee 1995). Sze and Lee also measured mucus secretion at 0.5 month, similar to the time frame used in this study. Their results indicated that mucus production in bivalves exposed to copper were elevated compared to copper-free controls for both species at temperatures of $17^{\circ} \mathrm{C}$ and $25^{\circ} \mathrm{C}$. At $25^{\circ} \mathrm{C}$, mucus secretion was highest at 0.5 month in comparison to all other time points.

Mucus production was also examined in relation to tissue copper concentration for individuals and treatment groups. In the 18 day experiment, a correlation between mean copper exposure and mucus/dry mass ratio was suggested but not statistically significant. Examination of the individual data points revealed that the individual measurements were highly variable, and this variation was consistent across exposure levels. The individual data points were more tightly and consistently grouped in the 12 day experiment, but also did not show a statistically significant relationship between copper exposure and mucus production.

One important difference between the two experiments is the number of specimens that were available at the end. The 18 day experiment had a much higher survivorship rate, especially in the High copper treatment, and thus included more mucus production data than the 12 day experiment. Differences in samples size, particularly when samples sizes are as small as those in this experiment, could 
potentially limit the power of statistical tests and result in significant results in one data set but not another.

\subsubsection{Algae Depletion}

No statistically significant relationships were observed in the algae depletion experiments, perhaps due to the small sample sizes used in this experiment and the high variability among measurements. Previous studies seem to suggest that the lack of statistical differences between exposure groups should not have been an unexpected result. A similar study measuring the effects of copper exposure on the mussel Perna perna did not show a statistically significant difference between filtration rates across copper concentrations similar to those in this study (Vosloo et al. 2012), although the published study was different in that the exposure period was only 24 hours and showed minimal variation in depletion rates between the control and the $50 \mathrm{ppb}$ groups. However, a more comparable study in which Perna perna were exposed to copper for a period of 24 days also showed a lack of statistically significant difference between a $50 \mathrm{ppb}$ concentration of copper and a control (Anandraj et al. 2002). The species used in these two studies was Perna perna, while some of the studies that showed a negative correlation between copper concentration and filtration rates used Mytilus edulis (e.g., Turner et al. 2009). This variability suggests that effects of copper on filtration rates may vary from species to species, and could explain the lack of significant correlations between copper and filtration rate in this study. It should be noted that Perna perna is considered an invasive species, and this lack of response to copper may 
be a mechanism that facilitates the spread of Perna perna and other non-indigenous species to contaminated environments (Anandraj et al. 2002).

Mytilus edulis, however, is not considered to be an invasive species (Turner et al. 2009), and an experiment with Mytilus edulis showed that copper exposure resulted in a significant reduction in algal depletion rate from the control, although there was no significant difference between copper exposure concentrations ranging from 18 to 56 ppb (Al-Subiai et al. 2011). In that study, the control clearance rate was much higher than that of even the lowest copper concentrations, and filtration rates were almost identical between mussels exposed to various concentrations of copper. Algae depletion was markedly reduced in copper exposure tests conducted on Ruditapes decussatus, which showed significant differences between the control and copper exposures of only $10 \mu \mathrm{L} \mathrm{L}^{-1}$, with rates reduced by up to $50 \%$ vs. the control (Sobral and Widdows 1997). Sze and Lee (2000) also showed a sharp drop in filtration rates for Perna viridis during exposure to $50 \mathrm{ppb}$ of copper.

\subsubsection{Native vs. Non-indigenous species.}

The crux of this study was to examine a native species, Chione californiensis, against a pair of non-indigenous species, Venerupis philippinarum and Musculista senhousia, that had been found in an overlapping environment in Mission Bay, San Diego, California, and to see how each responded to a copper-contaminated environment. Several studies have examined the competitive aspect of copper exposure and survival rates among native and non-indigenous species, but none had compared 
copper's sublethal effects, and none had examined the effects of copper on these specific species.

Some studies have directly examined the effects of copper pollution on the competitive balance between native and non-indigenous species. Crooks et al. (2011) showed that across a large suite of taxa, the number of native species exhibited a $40 \%$ decline as copper contamination increased, while the number of non-indigenous species did not change significantly. Similarly, Piola and Johnston (2008) found that copper contamination led to assemblages that had previously been occupied by mostly native species either being dominated by non-indigenous species or reduced to comparable numbers of native and non-indigenous species.

Specifically, with regard to bivalves, invasive species have been shown to be resistant to copper. The invasive bivalve species Mytilus galloprovincialis has been shown to resist antifouling practices, as it was found growing on surfaces coated with copper oxide and zinc oxide antifouling paints in sea chests on an Antarctic supply ship (Lee and Chown 2007).

The findings of these studies suggest that the non-indigenous species used in this experiment should have had a competitive advantage over the native Chione californiensis in terms of copper tolerance. Examination of the data from this experiment did not support those conclusions. The data generated from this study instead appears to support that among the three species tested, the indigenous Chione is better adapted to the conditions in Mission Bay in regard to copper concentration for the parameters that were tested, and may be better suited to withstand rising copper concentrations. The native Chione was shown to have a survivorship advantage over 
non-indigenous Musculista, and accumulated a significantly lower amount of whole body copper compared to Musculista. Other sublethal measures did not indicate any consistent competitive advantage for the non-indigenous species, and as such, the results of this study do not support the idea that these particular non-indigenous species had a competitive advantage over the native species used in this study. 


\section{Chapter 3: Conclusions}

\subsection{Conclusions}

There are several important conclusions that can be presented from the results of this study. The result with the most impact is that there was, in fact, a significant difference in some measures between a native bivalve species and non-indigenous species. Chione californiensis had a measurable though not statistically significant advantage in survivorship and accumulated a significantly lower amount of whole body copper than Musculista, two factors that indicate a potential competitive advantage in a copper-contaminated environment. Examination of the literature strongly suggests that this is the case, as tissue copper accumulation was deemed to be the most important factor affecting survival in copper-contaminated conditions, even more than dissolved copper concentrations in the environment (Sunila 1981, Sunila and Lindstrom 1985, Widdows and Johnson 1988, Grout and Levings 2001).

Survivorship and whole body copper accumulation were two measures that showed statistical significance. Most of the measures thought to be indicative of copper toxicity showed no statistically significant differences between species consistently across both experiments. The main patterns that were observed consistently across the experiments concerned survivorship, which in both studies showed decreases as copper exposure levels increased for all species, and copper accumulation for whole body tissue and gill tissue, which increased as copper exposure level increased for Venerupis and Musculista over both experiments, and for Chione in the 18 day experiment.

Prior to conducting this study, Venerupis and Chione were considered to be more similar to each other than either was to Musculista, in terms of size and growth 
rate (Crooks 1998), and morphological similarities were observed during dissection of specimens over the course of the study. The results of this study reflected this similarity, and many statistical results indicated disparities between Musculista and one or both of the other two species. There was a significant difference in whole body copper accumulation between Chione and Musculista and between Venerupis and Musculista across both experiments, reinforcing the expectation that Musculista would respond to copper exposure differently from the other two species. In addition, Musculista has been shown to grow at a rate much faster than either Chione or Venerupis in previous studies (Crooks 2001), which is likely to have produced the significant difference in growth percentage in comparison to both of the other species.

Other studies provide useful guidance for examining the impact of copper on bivalve growth. One study in particular showed that in adult bivalves, scope for growth was negatively correlated with copper exposure (Sobral and Widdows 1997). Scope for growth might be a better metric for measuring the effects of copper on growth in the future to reduce the problem of slow growth and small linear growth increments over short experimental durations. Scope for growth is a predictor of growth rate that relies on assimilated energy of food consumption against energy lost from respiration and defecation (Sobral and Widdows 1997) and requires additional measurements, including respiration rates and energy composition of fecal material. Although it is more laborious to estimate, this metric would break down the potential for growth; account for individual processes that may be impacted by copper, such as respiration; and provide additional detail on how copper affects organisms. 
The other statistically significant results that aligned with those from published studies involved mucus production. Though the calculation of the mucus/dry mass ratio was intended to normalize mucus production data in relation to body size, the large difference in body size between Musculista and Venerupis may have played a role in the statistical differences between the two species. The mass of mucus collected was generally low and mostly similar across trials, which may have been the result of mucus not being a good indicator of copper stress for these particular species or of issues with the collection procedure. Also, even though there were statistically significant differences for both experiments, the results from the 12 day experiment contradicted those from the 18 day experiment. Based on previous research and the larger $\mathrm{N}$ values in the 18 day experiment (Appendix B, Table 5.1), the results from the 18 day experiment seem more reliable, and those indicate that copper exposure is linked to mucus production, as has been shown in other bivalves (Sze and Lee, 1995). However, it is impossible to state this conclusion with certainty without more testing.

Most of the parameters measured in this study did not show statistical significance in relation to tissue copper accumulation. The few instances where there was statistical significance were not consistent across both experiments. The negative relationship between gill copper concentration and survivorship was statistically significant for Chione in the 18 day experiment. However, small sample sizes in both experiments may have obscured other relationships between tissue copper concentrations and various sublethal measures.

One important result from this study was the observation of consistent differences between the High copper treatment group and other two exposure groups. 
In general, there were no statistically significant differences between the Low and Medium copper treatment groups, suggesting a copper concentration threshold between $\sim 23.1 \mathrm{ppb}$ and $\sim 44.1 \mathrm{ppb}$. The existence of such a threshold would explain why most of the measures for the Medium copper treatment groups produced results similar to those for the Low copper groups. In the future, more copper concentrations between $25 \mathrm{ppb}$ and $50 \mathrm{ppb}$ should be examined and tested to try and identify this threshold.

The hypothesis of a physiological threshold is interesting in that it is partially represented in the copper accumulation data as well. One of the main findings for this experiment was that concentrations of copper in the gills correlated more closely to exposure than copper in the digestive tract. Also, gill copper accumulation mirrored the patterns observed in whole body accumulation, indicating that the most representative tissue to examine for copper accumulation in these bivalves may be the gills. Thus, suspension-feeding bivalves may be more vulnerable to copper contamination than deposit-feeding bivalves. An interesting follow-up experiment would be to examine tissue accumulation between suspension-feeding species and deposit-feeding species.

Of the three research questions posed in this study, the results provided fairly straightforward, although nuanced, answers to all of them. With regard to the relationship between copper accumulation and sublethal measures, there were no consistent statistically significant relationships between tissue copper accumulation and sublethal measures across both experiments. When it came to comparing the relationship between tissue copper concentrations and sublethal measures for the nonindigenous vs. native species, there was too much variation to suggest a consistent significant difference between Chione and the two non-indigenous species. 
Given the differences observed in survivorship, the lack of significance of sublethal measures across both experiments made it difficult to speculate about the reason why Chione survived at a higher rate than the other species. A possible reason as to why there was no consistent significant difference between Chione and the other two species in regard to most of the sublethal measures was low sample size. Especially at the highest levels of copper exposure, when the sublethal effects seemed most likely, the low number of surviving Musculista and Venerupis individuals reduced the power of statistical tests, compared to Chione, which had a larger pool of survivors to draw from. In a future experiment that generated higher numbers of survivors among the species, it is possible that correlations between copper concentrations and sublethal measures would be more clearly apparent.

The answer to how environmental copper affected copper accumulation is also fairly straightforward. On the whole, there was statistically significantly higher copper accumulation for whole body tissue, gill tissue, and the digestive tract tissue with increasing dissolved copper exposure levels. Statistically significant differences in whole body copper were observed between species for both experiments. However, differences between species for other tissues were not consistent across experiments, e.g., gill copper accumulation in Venerupis was significantly lower than the other species in the 12 day experiment but not in the 18 day experiment.

There was one consistent significant result with respect to tissue copper concentration across both experiments: Musculista was shown to accumulate significantly more whole body copper than either Chione or Venerupis. Coupled with the low survivorship of Musculista in comparison to Chione at higher copper concentrations 
(Figure 3.1), these results suggest that Musculista is affected more negatively by copper than Chione. Venerupis, on the other hand, had similarly low survivorship numbers but accumulated less whole body copper than Chione. Thus, copper accumulation and survivorship patterns differed between the two non-indigenous species in this study.

In terms of how the other sublethal measures reflected copper effects on each species and whether any evidence suggests a competitive advantage, the data simply do not support it. There were only a few measures that showed statistical differences between species, and there may be explanations for those statistical differences other than copper effects. There were statistical differences in growth among species, but the fact that growth rates were not correlated with dissolved copper concentration and that the difference was primarily due to Musculista, which has been shown to grow faster than the other two species in uncontaminated conditions, suggested that the differences in growth rate between species may not have been affected by copper. The statistical difference in mucus production between Chione and Musculista for the 12 day experiment is harder to explain. However, the same result was not obtained in the 18 day experiment, in which sample size for Musculista was larger.

Based on the results of this study, the null hypothesis that there is no significant difference in sublethal responses to copper between the native and non-indigenous species can be rejected, since Chione and Musculista showed significant differences in whole body copper across both experiments. Thus, although Chione differed from Musculista, the same was not true for Venerupis, and the data from this study are not sufficiently clear to conclude that this native species has a competitive advantage over the two non-indigenous species. However, it is worth noting that Chione exhibited by far 
the highest survival percentage for the High copper treatment group, although this difference wasn't apparent in the Medium copper treatment. The sublethal measures tested in this study didn't offer any conclusive explanation for this result. Chione accumulated significantly more whole body and gill copper per dry mass than Venerupis in the 12 day experiment, but this difference was not observed in the 18 day experiment. Whether increased tissue copper concentrations reflect increased harm or increased tolerance in Chione has yet to be determined.

The reason why Chione was able to survive the experimental conditions in greater numbers than the other two species is not immediately clear, as most of the literature on native vs. non-indigenous species suggest that non-indigenous species have survivorship advantages in contaminated environments, including those contaminated by copper (Crooks et al. 2011, McKenzie et al. 2011). As one of the few native bivalve species that continue to survive in Mission Bay, which has exhibited areas of high copper concentration (Kaufmann et al. 2004), it is possible that the success of Chione in this environment is reflective of a resistance to copper exposure. Also, it should be noted that the success of Venerupis and Musculista in Mission Bay may be a result of faster reproduction rates (Crooks 2001, Burnaford et al. 2011), and these species may not have the tolerance for contaminants like copper that are characteristic of some other non-indigenous species (Crooks 1998, Piola and Johnston 2008, 2009, Piola et al. 2009). 


\subsubsection{Limitations and Future Research}

There were several limitations to this study that could be addressed in the future. Perhaps the most influential limitation on the various experiments was small sample size. This aspect of the experiment was dictated by the availability of animals, and it was impossible to find an adequate number of specimens of each species in the same area. In theory, the number of specimens collected should have provided an adequate sample size without accounting for mortality. Low survivorship, however, was a key component to this study, and mortality in the High copper treatment groups severely impacted the number of surviving bivalves. Future studies will either need to use more specimens at the copper concentrations used in this study or consider reducing dissolved copper concentrations to generate a larger number of survivors. A pilot study would be needed to examine the survivorship threshold, maximizing sublethal responses to copper while at the same time lowering mortality rates from those observed in this study.

One of the other major limitations to this study was the time frame. Based on survival and growth rates, it's clear that the time frame was too short to be an adequate growth study based on the known growth rate of Chione (Peterson 1982) and too long at the dissolved copper concentrations to facilitate sufficient survivorship to conduct mucus production measurements at the end of the experiment. In retrospect, it may have been better to either not measure growth rates or lower the copper concentration to levels that would not have produced the amount of mortality that this study did. To maximize the number of surviving specimens at the end of an experiment, one of two things would need to change: either the number of specimens collected for testing 
would have to increase substantially, or the copper concentrations used in the experiment would have to be lowered. As mortality rates were as high as $93.3 \%$ for Musculista in the High copper treatment group, the best option would likely be to reduce the highest copper concentration. Based on the survivorship rates of this experiment, especially with the potential for a survivorship threshold between $23.1 \mathrm{ppb}$ and $44.1 \mathrm{ppb}$, lowering the designed copper concentrations to 0,15 , and $30 \mathrm{ppb}$ would likely increase survivorship and thus allow for more accurate evaluation of sublethal factors.

Another limitation of the experiment was dissolved copper concentrations that were different from those for which the study was designed. This limitation appears in several forms: inconsistency between experiments in dissolved copper concentrations, depletion of copper over time in the experimental tanks, and measured copper concentrations different from those that were calculated (Figure 1.3). To correct issues with copper concentration inconsistency and depletion there would have to be a more frequent source of copper, with new water regularly being introduced to ensure that the concentration of copper remains consistent over the course of the entire experiment. This procedure would ensure that all tanks at a particular exposure level remain at the same concentration and eliminate some of the variation between tanks. A manageable option would be to make small partial water changes at more frequent intervals than those used in this study. Another factor worth studying in the future would be to measure copper in terms of the free copper ion, instead of measuring total dissolved copper, as was done in this study. Free copper is the most dangerous form of copper to marine organisms (e.g., Zirino et al. 1998), and measurement of free copper concentration as a measure of availability in the environment and the effects of free 
copper on these species would more clearly illustrate the effects of increasing copper concentrations in the environment.

Future studies might want to consider eliminating some of the measures examined in this study and introducing others that were not measured. One test that was discussed but not implemented for logistical reasons was oxygen consumption, which would be worthwhile for a future study. Oxygen consumption suppression has been observed in the past as an indicator of copper-induced stress (Anandraj et al. 2002), and could provide useful information on how copper affects these species of bivalves. Filtration rate and growth rate could probably be removed from future studies, as growth was too slow for the experimental time frame, and measurements of filtration rate were time-intensive and produced highly variable results. Future research needs not be limited to the species used in this study. The hypotheses addressed here are applicable to any number of native and non-indigenous species, and the scale of the experiment could be changed to include even more species to get a better representation of how copper can affect various bivalves.

Another change for future studies would be the further examination of copper accumulation in tissues to examine critical thresholds for these species. This study was limited in that it was only designed to examine tissue accumulation by the surviving bivalves at the end of the experiment. Not only did this approach severely limit the sample size at the end of the experiment, particularly in the High copper treatment groups, but it represented a missed opportunity to learn more about tissue copper concentrations at the time of death. To better understand the relationship between survival and copper for these species, a future experiment examining and comparing 
tissue copper accumulation at time of death in comparison to that in survivors would be useful. Such an experiment would provide context for the survivors of this experiment and perhaps help to explain why they survived: are they simply tolerating higher tissue copper concentrations and surviving in spite of them, or are they more effectively removing copper from their tissues compared to individuals that died?

Another important factor that would be very useful in determining the impact of copper on the success of these species is the effect of environmental copper on the larvae of these species. All of the testing in this study involved adult specimens, and the results provide a good indication of how copper affects established individuals. A study of the impact of copper on larvae, however, would be invaluable in further examining the long-term impact of copper on bivalves in Mission Bay. Other important factors that could also be studied in the future include the effects of copper on the various aspects of reproduction, and the interaction between copper and other environmental factors.

Overall, the data from this experiment revealed significant differences that had implications in regards to potential competitive advantages between the species observed in regards to copper exposure. While most sublethal effects did not show differences between the species, the whole body copper accumulation did indicate a significant difference between Musculista and both Chione and Venerupis. Coupled with the fact that Chione had the highest survivorship amongst the tested species and there were no significant relationships between other sublethal factors and survivorship, the data generated from this experiment suggest that the native Chione may be better suited to surviving in a copper contaminated environment than the nonindigenous Musculista and Venerupis. 


\subsection{Literature Cited}

Absil, M.C.P., M. Berntssen and L.J.A. Gerringa. 1996. The influence of sediment, food and organic ligands on the uptake of copper by sediment-dwelling bivalves. Aquatic Toxicology 34: 13-29.

Ahn, I., J. Kang, and K. Kim. 2001. The effect of body size on metal accumulations in the bivalve Laterna elliptica. Antarctic Science 13: 355-362.

Al-Subiai, S.N., A.J. Moody, S.A. Mustafa, and A.N. Jha. 2011. A multiple biomarker approach to investigate the effects of copper on the marine bivalve mollusk Mytilus edulis. Ecotoxicology and Environmental Safety 74: 1913-1920.

Anandraj, A., D.M. Marshall, M.A. Gregory, and T.P. McClurg. 2002. Metal accumulation, filtration and $\mathrm{O}_{2}$ uptake rates in the mussel Perna perna (Mollusca: Bivalvia) exposed to $\mathrm{Hg}^{2+}, \mathrm{Cu}^{2+}$ and $\mathrm{Zn}^{2+}$. Comparative Biochemistry and Physiology Part C: Toxicology and Pharmacology 132: 355-363.

Burnaford, J.L., S.Y. Henderson, and B. Pernet. 2011. Assemblage shift following population collapse of a non-indigenous bivalve in an urban lagoon. Marine Biology 158: 1915-1927.

Byers, J. E. 2005. Marine reserves enhance abundance but not competitive impacts of a harvested nonindigenous species. Ecology 86: 487-500.

Carlton, J.T. 1979. History, biogeography, and ecology of the introduced marine and estuarine invertebrates of the Pacific coast of North America. PhD Dissertation. University of California, Davis, CA. 904 pp. 
Clark, G.F. and E.L. Johnston. 2005. Manipulating larval supply in the field" a controlled study of marine invasibility. Marine Ecology Progress Series 289: 9-19.

Crooks, J.A. 1998a. Habitat alteration and community-level effects of an exotic mussel, Musculista senhousia. Marine Ecology Progress Series 162: 137-152.

Crooks, J.A. 1998b. The Effects of the Introduced Mussel, Musculista senhousia, and Other Anthropogenic Agents on Benthic Ecosystems of Mission Bay, San Diego. Ph.D. Dissertation, University of California, San Diego, CA. 223 pp.

Crooks, J.A. 2001. Assessing invader roles within changing ecosystems: historical and experimental perspectives on a non-indigenous mussel in an urbanized lagoon. Biological Invasions 3: 23-36.

Crooks, J.A. and A. Suarez. 2006. Hyperconnectivity, NIS and the global breakdown of natural barriers to dispersal. Connectivity Conservation: Maintaining Connections for Nature. Cambridge University Press, Cambridge, pp. 451-578.

Crooks, J.A., A.L. Chang, and G.M. Ruiz. 2011. Aquatic pollution increases the relative success of NIS. Biological Invasions 13: 165-176.

Dexter, D.M, and J.A. Crooks. 2000. Benthic communities and the introduction of an exotic mussel in Mission Bay, San Diego: a long-term history. Bulletin of the Southern California Academy of Sciences 99: 128-146.

Drake, J.M. and J.M. Bossenbroek. 2009. Profiling ecosystem vulnerability to invasion by zebra mussels with support vector machines. Theoretical Ecology 2: 189-198. 
D'Silva, C. and T.W. Kureishy. 1978. Experimental studies on the accumulation of copper and zinc in the green mussel. Marine Pollution Bulletin 9: 187-190.

Elton, C.S. 1958. The Ecology of Invasions by Animals and Plants. Wiley, London. 196 pp. Environmental Protection Agency. 1996. Method 3052 Microwave Assisted Digestion of Siliceous and Organically Based Materials. 3052-1 Revision 0.

Garcia-Dominguez, F., G. Garcia-Melgar, and P. Gonzalez-Ramirez. 1993. Reproductive cycle of the clam Chione californiensis in Bahia Magdalena, Baja California Sur, Mexico. Ciencias Marinas 19: 12-28.

Gregory, M.A., D.J. Marshall, R.C. George, A. Anandraj, and T.P. McClurg. 2002. Correlations between metal uptake in the soft tissue of Perna perna and gill filament pathology after exposure to mercury. Marine Pollution Bulletin 45:1-12.

Grout, J.A., and C. D. Levings. 2001. Effects of acid mine drainage from an abandoned copper mine, Britannia Mines, Howe Sound, British Columbia, Canada, on transplanted blue mussels (Mytilus edulis). Marine Environmental Research 51: 265-288.

Heywood, V. 1995. Global Biodiversity Assessment. Cambridge University Press, Cambridge, UK. 1140 pp.

Hicks, D.W., Tunnell, J.W., McMahon, R.F., 2001. Population dynamics of the nonindigenous brown mussel Perna perna in the Gulf of Mexico compared to other world-wide populations. Marine Ecology Progress Series 211: 181-192. 
Johnston, E.L., R.F. Piola, and G.F. Clark. 2009. The role of propagule pressure in invasion success. Biological invasions in marine ecosystems: ecological management and geographic perspectives (ed. by G. Rilov and J. Crooks) Springer-Verlag, Berlin. pp. 131151.

Kaufmann, R.S., B.C. Stransky, J. Rudolph, D.T. Elliott, R.B. Griggs, J.N. Kittinger, B.L. Swope, J.P. Bolender, M.A. Boudrias and H.A. Sarabia. 2004. Mission Bay Water and Sediment Testing Project. Final Report. City of San Diego Agreement 2954-95. 101 pp.

Kaufmann, R.S., B.C. Stransky, D.T. Elliott, E. Kraak, D. Twal and K. Watters. 2006. Mission Bay Citizen Water Quality Monitoring and Education Program. Final Report. State Water Resources Control Board. Contract \#02-184-559-1. 12 pp.

Largier, J.L., J.T. Hollibaugh, and S.V. Smith. 1997. Seasonally hypersaline estuaries in Mediterranean-climate regions. Estuarine, Coastal, and Shelf Science 45: 789-797.

Lee, J.E. and S.L. Chown. 2007. Mytilus on the move: transport of an invasive bivalve to the Antarctic. Marine Ecology Progress Series 339: 307-310.

Lenz, M., B.A.P da Gama, N.V. Gerr, J. Gobin, F. Gröner, A. Harry, S.R. Jenkins, P. Kraufvelin, C. Mummelthei, J. Sareyka, E.A. Xavier, and M. Wahl. 2011. Non-indigenous marine invertebrates are more tolerant towards environmental stress than taxonomically related native species: results from a globally replicated study. Environmental Research 111: 943-952.

Lewis, A. G. 1995. Copper in Water and Aquatic Environments. International Copper Association, LTD. New York, NY. 65 pp. 
Lockwood, B.L., and G.N. Somero. 2011. Invasive and native blue mussels (genus Mytilus) on the California Coast: the role of physiology in a biological invasion. Journal of Experimental Biology and Ecology 400: 167-174.

Martínez-Córdova, L. R. 1988. Bioecología de la almeja negra Chione fluctifraga (Sowerby 1853). Revista de Biología Tropical 36: 213-219.

Martínez-Córdova, L. R., J.A. López, M. Martínez-Porchas, T. Bernal-Jaspeado, and A. Miranda-Baeza. 2011. Studies on the bioremediatal capacity of the adult black clam, Chione fluctifraga, of shrimp culture effects. Journal of Marine Biology and Oceanography (Revista de Biologia Marina y Oceanografía) 46: 105-113.

McKenzie, L.A., R. Brooks, and E.L. Johnston. 2011. Heritable pollution tolerance in an marine invader. Environmental Research 111: 926-932.

Montgomery, W.I., M.G. Lundy, and N. Reid. 2012. 'Invasional meltdown': evidence for unexpected consequence and cumulative impacts of multispecies invasions. Biological Invasions 14: 1111-1125.

Nadella, S.R., J.L. Fitzpaterick, N. Franklin,C. Bucking, D.S. Smith, C.M. Wood. 2009. Toxicity of $\mathrm{Cu}, \mathrm{Zn}, \mathrm{Ni}$, and $\mathrm{Cd}$ to developing embryos of the blue mussel (Mytilus trossolus) and the protective effect of dissolved organic carbon. Comparative Biochemistry and Physiology 149: 340-348.

Neira, C., F. Delgadillo-Hinokosa, A. Zirino, G. Mendoza, L.A. Levin, M. Porrachia, and D. D. Dehyn. 2009. Spatial distribution of copper in relation to recreational boating in a California shallow-water basin. Chemistry and Ecology 25: 417-433. 
Peterson, C. 1982. The importance of predation and intra- and interspecific competition in the population biology of two infaunal suspension-feeding bivalves, Protothaca staminea and Chione undatella. Ecological Monographs 52: 437-475.

Pillay, K. 2013. Mucus secretion responses to various sublethal copper (II) concentrations in the mussel Perna perna. International Journal of Environmental and Ecological Engineering 7: 225-228.

Piola, R.F. and E.L. Johnston. 2008. Pollution reduces native diversity and increases invader dominance in marine hard-substrate communities. Diversity and Distributions 14: $329-342$.

Piola, R.F. and E.L. Johnston. 2009. Comparing differential tolerance of native and nonindigenous species to metal pollution using novel assay techniques. Environmental Pollution 157: 2853-2864.

Piola, R.F., K.A. Dafforn, and E.L. Johnston. 2009. The influence of antifouling practices on marine invasions. Biofouling 25: 633-644.

Pyle, G.G. and R.S. Mirza. 2007. Copper-impaired chemosensory function and behavior in aquatic animals. Human Ecology Risk Assessment 13: 492-505.

Reichelt-Brushett, A.J., and P.L. Harrison. 2000. The effects of copper on the settlement success of larvae from the Scleractinian coral Acroporatenuis. Marine Pollution Bulletin 41: 385-391. 
Rejmanek, M. 1989. Invasibility of plant communities. in J. A. Drake, H. A. Mooney, F. di Castri, R. H. Groves, F. J. Kruger, M. Rejmanek, and M. Williamson, editors. Biological Invasions: A Global Perspective, SCOPE 37. John Wiley, New York, New York, USA. pp. 369-383.

Reusch, T.B.H. 1998. Native predators contribute to invasion resistance to the nonindigenous bivalve Musculista senhousia in southern California, USA. Marine Ecology Progress Series 170: 159-168.

Richardson, D.M. and P. Pysek. 2006. Plant invasions: merging the concepts of species invasiveness and community invasibility. Progress in Physical Geography 30: 409-431.

Rivera-Duarte, I. and A. Zirino. 2004. Respone of the $\mathrm{Cu}$ (II) Ion Selective Electrode to $\mathrm{Cu}$ Titration in Artificial and Natural Shore Seawater and in the Measurement of the $\mathrm{Cu}$ Complexation Capacity. Environmental Science Technology 38: 3139-3147.

Rygg, B. 1985. Effect of sediment copper on benthic fauna. Marine Ecology Progress Series 25: 83-89.

Salviati, L., E. Hernandez-Rosa, W.F. Walker, S. Sacconi, S. Dimauro, E.A. Sachon, and M.M. Davidson. 2002. Copper supplementation restores cytochrome c oxidase activity in cultured cells from patients with $\mathrm{SCO}_{2}$ mutations. Biochemistry Journal 363: 321-327.

Schiff, K., D. Diehl, and A. Valkirs. 2004. Copper emissions from antifouling paint on recreational vessels. Marine Pollution Bulletin 48: 371-377. 
Scott, D.M. and C.W. Major. 1972. The effect of Copper (II) on Survival, Respiration, and Heart Rate in the Common Blue Mussel Mytilus edulis. Biological Bulletin 143: 679-688.

Simberloff, D. and B. Von Holle. 1999. Positive interactions of nonindigenous species: invasional meltdown? Biological Invasions 1: 21-32.

Sobral, P., and J. Widdows. 1997. Effects of copper exposure on the scope for growth of the clam Ruditapes decussatus from southern Portugal. Marine Pollution Bulletin 34: $992-1000$.

Strom, D., S. Simpson, G. Batley, and D. Jolley. 2011. The influence of sediment particle size and organic carbon on the toxicity of copper to benthic invertebrates in oxic/suboxic surface sediments. Environmental Toxicology and Chemistry 30: 15991610.

Sunda, W.G., P.A. Tester, and S.A. Huntsman. 1987. Effects of cupric and zinc ions on the survival and reproduction of marine copepods. Marine Biology 94: 203-210.

Sunila, I. 1981. Toxicity of copper and cadmium to Mytilus edulis L. (Bivalvia) in brackish water. 1981. Annales Zoologici Fennici 18: 221-223.

Sunila, I. and R. Lindstrom. 1985. Survival, growth and shell deformities of copper- and cadmium-exposed mussels (Mytilus edulis L.) in brackish water. Estuarine, Coastal, and Shelf Science 21: 555-565.

Sze, P.W.C and S.Y. Lee. 1995. The potential role of mucus in the depuration of copper from the mussels Perna viridis and Septifer virgatus. Marine Pollution Bulletin 31: 390393. 
Sze, P.W.C and S.Y. Lee. 2000. Effects of copper on the green mussel Perna viridis. Marine Biology 137: 379-392.

Taylor, L.N., C. M. Wood, and D.G. McDonald. 2003. An evaluation of sodium loss and gill metal binding properties in rainbow trout and yellow perch to explain species differences in copper tolerance. Environmental Toxicology and Chemistry 22: 21592166.

Trannum, H.C., F. Olsgard, J.M. Skei, J. Indrehus, S. Overas, and J. Eriksen. 2004. Effects of copper, cadmium, and contaminated harbor sediments on recolonisation of softbottom communities. Journal of Experimental Marine Biology and Ecology 310: 87-114.

Troost, K. 2011. Causes and effects of a highly successful marine invasion: Case-study of the introduced Pacific oyster Crassostrea gigas in continental NW European estuaries. Journal of Sea Research 64: 145-165.

Turner, A., M. Barrett, and M.T. Brown. 2009. Processing of antifouling paint particles by Mytilus edulis. Environmental Pollution 157: 215-220.

Tuskes, P. 2012. Survey of Mission Bay Mollusks, San Diego, California. The Festivus 64: $13-28$.

Verbrugge, L.N.H., A.M. Schipper, M.A.J. Huijbregts, G. Van der Velde, and R. S.E.W. Leuven. 2012. Sensitivity of native and non-indigenous mollusc species to changing river water temperature and salinity. Biological Invasions 14: 1187-1199. 
Vosloo, D., J. Sara, and A. Vosloo. 2012. Acute responses of brown mussel (Perna perna) exposed to sub-lethal copper levels: Integration of physiological and cellular responses. Aquatic Toxicology 106-107: 1-8.

Waykar, B. and S.M. Shinde. 2011. Assessment of the metal bioaccumulation in three species of freshwater bivalves. Bulletin of Environmental Contamination and Toxicology 87: $267-271$.

Wilson, J.D., M.E. Dorcas, and R.W. Snow. 2011. Identifying plausible scenarios for the establishment of the invasive Burmese python (Python molarus) in Southern Florida. Biological Invasions 13: 1493-1504.

Widdows, J. 1985. Physiological responses to pollution. Marine Pollution Bulletin 16: 129-134.

Widdows, J. and D. Johnson. 1988. Physiological energetics of Mytilus edulis: scope for growth. Marine Ecology Progress Series 46: 113-121.

Wright, M.G., M. P. Hoffman, T.P. Kuhlar, J. Gardner, and S.A. Pitcher. 2005. Evaluating the risks of biological introductions: a probabilistic risk-assessment approach. Biological Control 35: 338-347.

Zirino, A., S.L. Belli, and D.A. Van der Weele. 1998. Copper Concentrations and $\mathrm{Cu}^{\mathrm{ii}}$ Activity in San Diego Bay. Electroanalysis 10: 423-427. 


\section{APPENDIX A: Mission Bay}

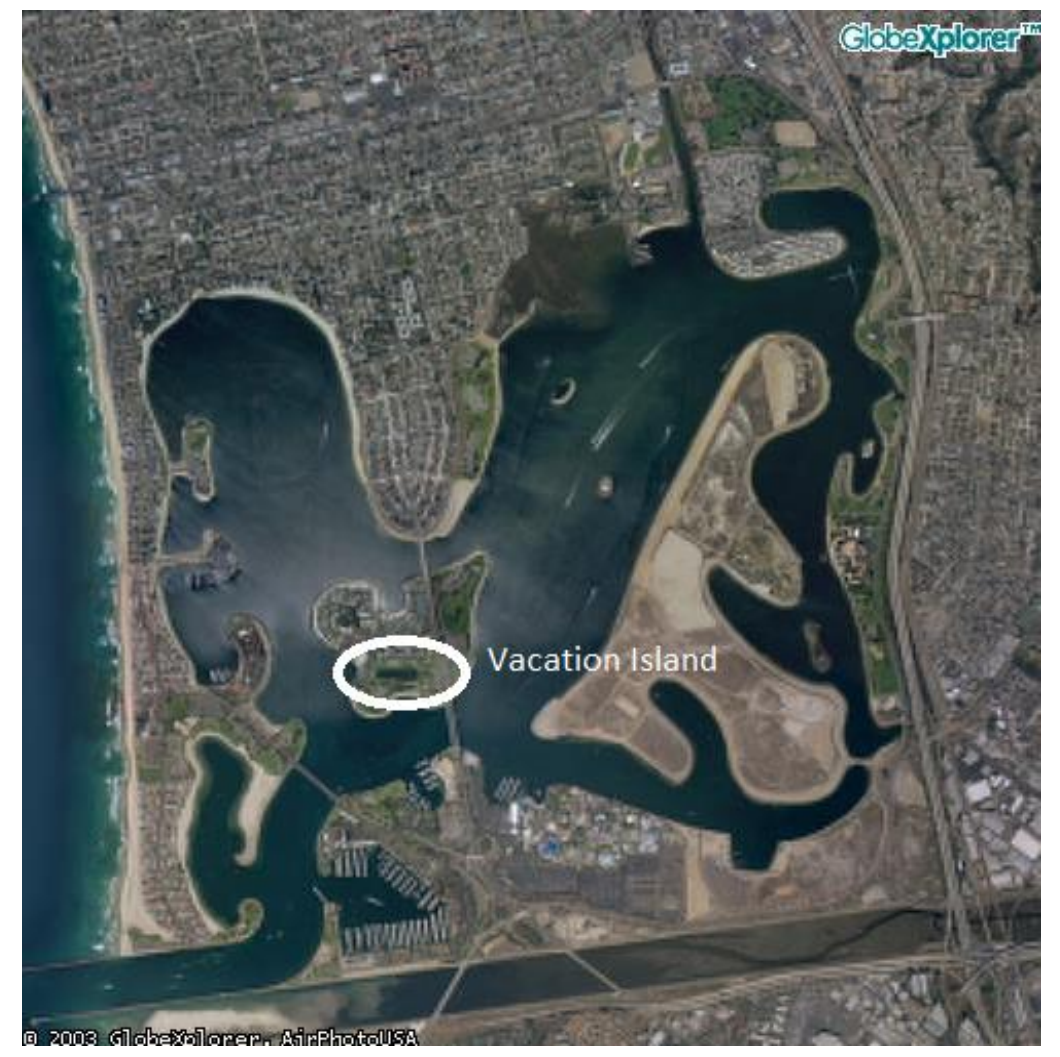

Figure $A$

Above: Map of Mission Bay with sampling site on Vacation Island marked.

Source: http://home.sandiego.edu/ kaufmann/MissionBay/MissionBayPhoto.jpg 
A local ecosystem that has been impacted both by anthropogenic pollution and the presence of non-indigenous species is Mission Bay in San Diego, California (Figure A). Mission Bay is an area of human-generated disturbance, due to its use as a popular recreation park (Crooks 2001). Physical disturbance of the environment through boating and other human activity is common in recreational water parks such as Mission Bay, and disturbed environments typically exhibit weakened biotic resistance due to the impact of disturbance on native species (Piola et al. 2009). Clark and Johnston (2005) found that physical disturbance was a mediating factor in the establishment of invasive bryozoan species. This physical disturbance was impact-based, with an example being a boat striking a settlement surface. San Diego Bay, a nearby body of water that undergoes a high level of human use, showed a clear correlation between boating activity and dissolved copper concentrations in different parts of the bay (Neira et al. 2009).

A flushing gradient has been observed within Mission Bay, as indicated by increasing salinity with distance from the mouth during the summer (Largier et al. 1997). Areas near the creek mouths in the back of the bay are often poorly flushed in comparison to areas near the mouth of the bay, which exchange water tidally with the Pacific Ocean (Largier et al. 1997). Circulation in the bay is impacted by seasonal differences in rainfall, with more runoff during the winter and very little in the summer (Largier et al. 1997). The flushing gradient also plays a role in the sediment grain size and pollution gradients in Mission Bay. The high-energy flushing of the front of the bay re-suspends finer sediments, allowing only larger, coarser grains to settle, while tidal mixing in the front of the bay dilutes concentrations of pollutants via exchange with the ocean. 
Sediment properties of Mission Bay indicate a gradient from coarser sediments in the front of the bay to finer sediments in the back of the bay (Kaufmann et al. 2004). This pattern is likely due to the fact that the back of the bay has many creek outlets that deposit fine-grained sediments, and that the residence time of the water in the back bay is higher than the front of the bay, leading to more particle deposition, in addition to the previously-mentioned resuspension in the front bay. Contamination gradients are likely the result of the flushing and sediment gradients. The poor circulation experienced in the back of the bay leads to a buildup of contaminants accumulated through runoff via the creek outlets and numerous storm drains that channel runoff into the bay and also results in long residence times for contaminants that enter the back of the bay. Finegrained sediments, such as those found in the back bay, also are better at sequestering metal contaminants, because finer grained sediments have a greater amount of surface area to which contaminants can adsorb (Zirino et al. 1998).

Due to circulation patterns, only the front part of the bay is well-flushed. The back of the bay, with little circulation and numerous creeks and storm drains emptying into it, tends to accumulate more pollutants (Kaufmann et al. 2004). An analysis of contaminant concentrations in Mission Bay sediment showed a clear gradient, as levels of pesticides, copper, lead, and zinc all clearly increased with distance from the mouth of the bay (Kaufmann et al. 2006). The areas with the highest contaminant loads of potentially toxic metals like zinc and copper were near the poorly circulated creek outlet areas near the back of the bay (Kaufmann et al. 2006). Contaminants in Mission Bay have been shown to vary based on seasonality and rainfall. In a report in 2004, it was shown that at different times of the year there were different copper and zinc concentrations in the water column (Kaufmann et al. 2004). Due to the presence of 
gradients for flushing, sediment properties, and contamination, Mission Bay is an excellent setting for this experiment, because it represents different variables across their ranges (Kaufmann et al. 2004). For example, Ventura Point, which is well flushed, showed a copper concentration of 16.6 ppb in May 2002, while Tecolote Creek, a back bay site with less flushing had water copper concentrations of $26.6 \mathrm{ppb}$ during the same sampling period (Kaufmann et al. 2004). As a result, Mission Bay can be used as an example of contamination research for a wide range of coastal environments. 


\section{Appendix B: Tables}

Table 1.1 Table depicting an example of the staggered layout and schedule of postdepuration experiment initiation.

\begin{tabular}{|c|c|c|c|c|}
\hline $\mathrm{Cu}$ & First day & Second day & Third day & Fourth day \\
\hline Low & $\begin{array}{ll}\text { Day } 1 & \\
\text { - } & \begin{array}{l}\text { Specimens } \\
\text { introduced to } \\
\text { tank }\end{array} \\
\text { - General Feeding } \\
\text { - Initiate first } \\
\text { algae depletion }\end{array}$ & $\begin{aligned} \text { Day } 2 & \\
& \\
& \text { Terminate first } \\
& \text { algae depletion }\end{aligned}$ & $\begin{array}{l}\text { Day } 3 \\
\text { - No action taken }\end{array}$ & $\begin{array}{l}\text { Day } 4 \\
\text { - General Feeding } \\
\text { - Initiate second } \\
\text { algae depletion }\end{array}$ \\
\hline Medium & & $\begin{array}{l}\text { Day } 1 \text { Specimens } \\
\begin{array}{l}\text { - } \\
\text { introduced to } \\
\text { tank }\end{array} \\
\text { - General } \\
\text { Feeding } \\
\text { - Initiate first } \\
\text { algae depletion }\end{array}$ & $\begin{array}{l}\text { Day } 2 \\
\text { - Terminate first } \\
\text { algae depletion }\end{array}$ & $\begin{array}{l}\text { Day } 3 \\
\text { - No action taken }\end{array}$ \\
\hline High & & & $\begin{array}{ll}\text { Day } 1 & \\
\text { - } & \begin{array}{l}\text { Specimens } \\
\text { introduced to } \\
\text { tank }\end{array} \\
\text { - General } \\
\text { Feeding }\end{array}$ & $\begin{array}{l}\text { Day } 2 \\
\begin{array}{l}\text { - Terminate first } \\
\text { algae depletion }\end{array}\end{array}$ \\
\hline
\end{tabular}


Table 2.1: Tissue copper data from both experiments for whole body, gills, and digestive tract. Means, standard deviations, variances, $\mathrm{N}$ values, and medians are presented for each species in each copper concentration treatment. 


\begin{tabular}{|c|c|c|c|c|c|c|c|c|c|}
\hline \multicolumn{5}{|c|}{18 Day } & \multicolumn{5}{|c|}{12 Day } \\
\hline \multicolumn{10}{|c|}{ Chione } \\
\hline \multicolumn{5}{|c|}{ Whole Body } & \multicolumn{5}{|c|}{ Whole Body } \\
\hline$\underline{\text { Tank }}$ & Mean $(\mu \mathrm{g} / \mathrm{g})$ & $\underline{\mathrm{N}}$ & $\underline{\text { Standard Dev. }}$ & Median & $\underline{\text { Tank }}$ & Mean $(\mu \mathrm{g} / \mathrm{g})$ & $\underline{\mathrm{N}}$ & Standard Dev. & Median \\
\hline Low Copper & 18.98 & 3 & 3.29 & 20.40 & Low Copper & 40.04 & 3 & 10.24 & 41.68 \\
\hline Medium Copper & 52.65 & 3 & 10.80 & 54.15 & Medium Copper & 99.44 & 3 & 7.01 & 95.68 \\
\hline High Copper & 85.83 & 3 & 20.40 & 85.67 & High Copper & 56.93 & 3 & 13.88 & 57.54 \\
\hline \multicolumn{5}{|c|}{ Gills } & \multicolumn{5}{|c|}{ Gills } \\
\hline Low Copper & 37.12 & 3 & 19.77 & 26.53 & Low Copper & 37.23 & 3 & 30.34 & 34.65 \\
\hline Medium Copper & 54.16 & 3 & 19.39 & 60.29 & Medium Copper & 84.67 & 3 & 23.51 & 81.43 \\
\hline High Copper & 106.16 & 3 & 69.80 & 70.52 & High Copper & 95.82 & 3 & 49.80 & 70.85 \\
\hline \multicolumn{5}{|c|}{ Digestive Tract } & \multicolumn{5}{|c|}{ Digestive Tract } \\
\hline Low Copper & 93.78 & 3 & 65.75 & 72.81 & Low Copper & 24.56 & 3 & 3.65 & 24.26 \\
\hline Medium Copper & 117.63 & 3 & 22.82 & 117.42 & Medium Copper & 87.43 & 3 & 28.47 & 89.37 \\
\hline High Copper & 102.80 & 3 & 31.91 & 90.52 & High Copper & 96.58 & 3 & 38.52 & 89.54 \\
\hline \multicolumn{10}{|c|}{ Venerupis } \\
\hline \multicolumn{5}{|c|}{ Whole Body } & \multicolumn{5}{|c|}{ Whole Body } \\
\hline$\underline{\text { Tank }}$ & Mean $(\mu \mathrm{g} / \mathrm{g})$ & $\underline{\mathrm{N}}$ & Standard Dev. & Median & $\underline{\text { Tank }}$ & Mean $(\mu \mathrm{g} / \mathrm{g})$ & $\underline{\mathrm{N}}$ & Standard Dev. & Median \\
\hline Low Copper & 23.01 & 3 & 12.11 & 17.53 & Low Copper & 31.11 & 3 & 15.15 & 23.79 \\
\hline Medium Copper & 42.26 & 3 & 19.06 & 31.73 & Medium Copper & 48.62 & 3 & 7.32 & 52.62 \\
\hline High Copper & 64.46 & 2 & 0.83 & 64.46 & High Copper & 58.06 & 1 & $\mathrm{~N} / \mathrm{A}$ & $\mathrm{N} / \mathrm{A}$ \\
\hline \multicolumn{5}{|c|}{ Gills } & \multicolumn{5}{|c|}{ Gills } \\
\hline Low Copper & 20.95 & 3 & 10.37 & 19.22 & Low Copper & 9.95 & 3 & 5.39 & 9.28 \\
\hline Medium Copper & 30.17 & 3 & 10.37 & 26.09 & Medium Copper & 41.40 & 3 & 13.08 & 35.46 \\
\hline High Copper & 128.56 & 2 & 22.56 & 128.56 & High Copper & 47.08 & 1 & N/A & 47.08 \\
\hline \multicolumn{5}{|c|}{ Digestive Tract } & \multicolumn{5}{|c|}{ Digestive Tract } \\
\hline \begin{tabular}{|l|} 
Low Copper \\
\end{tabular} & 44.63 & 3 & 33.06 & 44.89 & Low Copper & 92.88 & 3 & 41.43 & 98.38 \\
\hline Medium Copper & 132.16 & 3 & 61.37 & 127.65 & Medium Copper & 54.29 & 3 & 9.28 & 51.46 \\
\hline High Copper & 194.14 & 2 & 125.42 & 194.14 & High Copper & 58.40 & 1 & $\mathrm{~N} / \mathrm{A}$ & 58.40 \\
\hline \multicolumn{10}{|c|}{ Musculista } \\
\hline \multicolumn{5}{|c|}{ Whole Body } & \multicolumn{5}{|c|}{ Whole Body } \\
\hline$\underline{\text { Tank }}$ & Mean $(\mu \mathrm{g} / \mathrm{g})$ & $\underline{N}$ & Standard Dev. & Median & $\underline{\text { Tank }}$ & Mean $(\mu \mathrm{g} / \mathrm{g})$ & $\underline{\mathrm{N}}$ & Standard Dev. & Median \\
\hline Low Copper & 40.35 & 3 & 18.53 & 30.42 & Low Copper & 60.74 & 3 & 11.21 & 54.42 \\
\hline Medium Copper & 100.13 & 3 & 28.70 & 84.28 & Medium Copper & 181.81 & 3 & 52.01 & 153.00 \\
\hline High Copper & 194.87 & 1 & $\mathrm{~N} / \mathrm{A}$ & $\mathrm{N} / \mathrm{A}$ & High Copper & 202.20 & 1 & N/A & $\mathrm{N} / \mathrm{A}$ \\
\hline \multicolumn{5}{|c|}{ Gills } & \multicolumn{5}{|c|}{ Gills } \\
\hline Low Copper & 22.24 & 3 & 7.27 & 25.67 & Low Copper & 18.53 & 3 & 14.38 & 10.32 \\
\hline Medium Copper & 44.85 & 3 & 26.05 & 35.70 & Medium Copper & 186.49 & 3 & 80.06 & 183.57 \\
\hline High Copper & 176.32 & 2 & 76.74 & 176.32 & High Copper & $\mathrm{N} / \mathrm{A}$ & 0 & $\mathrm{~N} / \mathrm{A}$ & $\mathrm{N} / \mathrm{A}$ \\
\hline \multicolumn{5}{|c|}{ Digestive Tract } & \multicolumn{5}{|c|}{ Digestive Tract } \\
\hline Low Copper & 86.07 & 3 & 129.08 & 14.77 & Low Copper & 20.22 & 3 & 5.94 & 23.00 \\
\hline Medium Copper & 248.66 & 3 & 11.94 & 242.11 & Medium Copper & 171.94 & 3 & 70.19 & 136.45 \\
\hline High Copper & 62.85 & 2 & 32.89 & 62.85 & High Copper & $\mathrm{N} / \mathrm{A}$ & 0 & $\mathrm{~N} / \mathrm{A}$ & $\mathrm{N} / \mathrm{A}$ \\
\hline
\end{tabular}


Table 3.1: Compilation of descriptive statistics for survivorship by tank for Chione californiensis, Venerupis philippinarum, and Musculista senhousia for both the 18 day and 12 day experiments. The initial number of individuals of each species in each tank was 5. The Mean (Ratio) is the proportion of survivors, with 1.000 being $100 \%$. N represents the total number of surviving specimens.

\begin{tabular}{|c|c|c|c|c|c|c|c|}
\hline \multicolumn{7}{|c|}{18 Day } & \multicolumn{5}{c|}{12 Day } \\
\hline \multicolumn{7}{|c|}{ Chione } \\
\hline \multicolumn{7}{|c|}{ Survivorship By Tank } & \multicolumn{3}{c|}{ Survivorship By Tank } \\
\hline Tank & Mean (Ratio) & Standard Dev. & N & Tank & Mean (Ratio) & Standard Dev. & N \\
\hline Low Copper & 0.933 & 0.115 & 14 & Low Copper & 0.867 & 0.115 & 13 \\
\hline Medium Copper & 0.867 & 0.115 & 13 & Medium Copper & 1.000 & 0.000 & 15 \\
\hline High Copper & 0.733 & 0.115 & 11 & High Copper & 0.467 & 0.306 & 7 \\
\hline \multicolumn{7}{|c|}{ Venerupis } \\
\hline Survivorship By Tank & \multicolumn{7}{|c|}{ Survivorship By Tank } \\
\hline Tank & Mean (Ratio) & Standard Dev. & N & Tank & Mean (Ratio) & Standard Dev. & N \\
\hline Low Copper & 1.000 & 0.000 & 15 & Low Copper & 1.000 & 0.000 & 15 \\
\hline Medium Copper & 0.867 & 0.231 & 13 & Medium Copper & 0.800 & 0.200 & 12 \\
\hline High Copper & 0.267 & 0.115 & 4 & High Copper & 0.200 & 0.200 & 3 \\
\hline \multicolumn{7}{|c|}{ Musculista } \\
\hline Survivorship By Tank & \multicolumn{3}{|c|}{ Survivorship By Tank } \\
\hline Tank & Mean (Ratio) & Standard Dev. & N & Tank & Mean (Ratio) & Standard Dev. & N \\
\hline Low Copper & 0.933 & 0.115 & 14 & Low Copper & 1.000 & 0.000 & 15 \\
\hline Medium Copper & 0.933 & 0.115 & 14 & Medium Copper & 0.600 & 0.346 & 9 \\
\hline High Copper & 0.267 & 0.231 & 4 & High Copper & 0.067 & 0.115 & 1 \\
\hline
\end{tabular}


Table 4.1: Descriptive statistics for overall growth and growth percentage of Chione californiensis, Venerupis philippinarum, and Musculista senhousia. Data are presented only for the 12 day experiment, as described in the text. $\mathrm{N}$ represents the number of surviving bivalves.

\begin{tabular}{|c|c|c|c|c|c|c|c|}
\hline \multicolumn{8}{|c|}{ Chione } \\
\hline \multicolumn{4}{|c|}{ Overall Growth } & \multicolumn{4}{|c|}{ Growth Percentage } \\
\hline Tank & Mean $(\mathrm{mm})$ & Standard Dev. & $\mathrm{N}$ & Tank & Mean $(\%)$ & Standard Dev. & $\mathrm{N}$ \\
\hline Low Copper & 0.07 & 0.04 & 13 & Low Copper & 0.22 & 0.11 & 13 \\
\hline Medium Copper & 0.04 & 0.03 & 15 & Medium Copper & 0.12 & 0.08 & 15 \\
\hline High Copper & 0.06 & 0.04 & 7 & \begin{tabular}{|l|} 
High Copper \\
\end{tabular} & 0.18 & 0.12 & 7 \\
\hline \multicolumn{8}{|c|}{ Venerupis } \\
\hline \multicolumn{4}{|c|}{ Overall Growth } & \multicolumn{4}{|c|}{ Growth Percentage } \\
\hline Tank & Mean $(\mathrm{mm})$ & Standard Dev. & $\mathrm{N}$ & Tank & Mean $(\%)$ & Standard Dev. & $\mathrm{N}$ \\
\hline Low Copper & 0.04 & 0.03 & 15 & Low Copper & 0.11 & 0.08 & 15 \\
\hline Medium Copper & 0.05 & 0.02 & 12 & Medium Copper & 0.14 & 0.07 & 12 \\
\hline High Copper & 0.05 & 0.02 & \begin{tabular}{|l|l}
3 & \\
\end{tabular} & \begin{tabular}{|l|} 
High Copper \\
\end{tabular} & 0.13 & 0.06 & 3 \\
\hline \multicolumn{8}{|c|}{ Musculista } \\
\hline \multicolumn{4}{|c|}{ Overall Growth } & \multicolumn{4}{|c|}{ Growth Percentage } \\
\hline Tank & Mean $(\mathrm{mm})$ & Standard Dev. & $\mathrm{N}$ & Tank & Mean (\%) & Standard Dev. & $\mathrm{N}$ \\
\hline Low Copper & 0.08 & 0.12 & 15 & \begin{tabular}{|l|} 
Low Copper \\
\end{tabular} & 0.51 & 0.79 & 15 \\
\hline Medium Copper & 0.05 & 0.03 & 9 & Medium Copper & 0.32 & 0.16 & 9 \\
\hline High Copper & 0.07 & N/A & 1 & High Copper & 0.44 & N/A & 1 \\
\hline
\end{tabular}


Table 5.1: Compilation of statistics for mucus/dry mass ratio of Chione californiensis, Venerupis philippinarum, and Musculista senhousia. $\mathrm{N}$ represents number of individuals tested.

\begin{tabular}{|c|c|c|c|c|c|c|c|c|c|}
\hline \multicolumn{5}{|c|}{ Day 18} & \multicolumn{5}{|c|}{ Day 12} \\
\hline \multicolumn{10}{|c|}{ Chione } \\
\hline \multicolumn{5}{|c|}{ Mucus/Dry Mass } & \multicolumn{5}{|c|}{ Mucus/Dry Mass } \\
\hline Tank & Mean & $\underline{N}$ & Standard Dev. & Median & Tank & Mean & $\underline{N}$ & Standard Dev. & Median \\
\hline Low Copper & 0.184 & 7 & 0.209 & 0.069 & Low Copper & 0.485 & 6 & 0.140 & 0.457 \\
\hline Medium Copper & 0.085 & 6 & 0.080 & 0.048 & Medium Copper & 0.359 & 7 & 0.138 & 0.311 \\
\hline High Copper & 0.385 & 6 & 0.251 & 0.416 & High Copper & 0.058 & 3 & 0.022 & 0.053 \\
\hline \multicolumn{10}{|c|}{ Venerupis } \\
\hline \multicolumn{5}{|c|}{ Mucus/Dry Mass } & \multicolumn{5}{|c|}{ Mucus/Dry Mass } \\
\hline Tank & Mean & $\underline{N}$ & Standard Dev. & Median & Tank & Mean & $\underline{N}$ & Standard Dev. & Median \\
\hline Low Copper & 0.110 & 7 & 0.123 & 0.043 & Low Copper & 0.223 & 7 & 0.116 & 0.160 \\
\hline Medium Copper & 0.090 & 6 & 0.143 & 0.022 & Medium Copper & 0.348 & 5 & 0.154 & 0.325 \\
\hline High Copper & 0.111 & 2 & 0.084 & 0.111 & High Copper & 0.016 & 1 & $\mathrm{~N} / \mathrm{A}$ & 0.016 \\
\hline \multicolumn{10}{|c|}{ Musculista } \\
\hline \multicolumn{5}{|c|}{ Mucus/Dry Mass } & \multicolumn{5}{|c|}{ Mucus/Dry Mass } \\
\hline Tank & Mean & $\underline{N}$ & Standard Dev. & Median & Tank & Mean & $\underline{N}$ & Standard Dev. & Median \\
\hline Low Copper & 0.242 & 7 & 0.189 & 0.285 & Low Copper & 0.143 & 7 & 0.108 & 0.095 \\
\hline Medium Copper & 0.184 & 7 & 0.092 & 0.165 & Medium Copper & 0.109 & 3 & 0.119 & 0.041 \\
\hline High Copper & 0.488 & 2 & 0.280 & 0.488 & High Copper & 0.000 & 1 & $\mathrm{~N} / \mathrm{A}$ & 0.000 \\
\hline
\end{tabular}


Table 6.1: Algae depletion statistics for Chione californiensis, Venerupis philippinarum, and Musculista senhousia. Positive values indicate lower algal measured cell densities at the end of each trial compared to initial densities. Negative values indicate higher measured cell densities at the end of the trial.

\section{A.) 18 day experiment}

B.) 12 day experiment 


\begin{tabular}{|c|c|c|c|c|c|c|c|c|c|}
\hline \multicolumn{10}{|c|}{18 Day } \\
\hline \multicolumn{5}{|c|}{ Day 0} & \multicolumn{5}{|c|}{ Day 17} \\
\hline \multicolumn{10}{|c|}{ Control } \\
\hline \multicolumn{5}{|c|}{ Algae Depletion } & \multicolumn{5}{|c|}{ Algae Depletion } \\
\hline Tank & Mean & $\underline{\mathrm{N}}$ & Standard Dev. & Median & Tank & Mean & $\underline{\mathrm{N}}$ & Standard Dev. & Median \\
\hline Low Copper & 0.012 & 2 & 0.011 & 0.012 & \begin{tabular}{|l|} 
Low Copper \\
\end{tabular} & -0.198 & 2 & \begin{tabular}{|l|}
0.013 \\
\end{tabular} & -0.198 \\
\hline Medium Copper & -0.080 & 2 & 0.012 & -0.080 & Medium Copper & -0.191 & 2 & 0.115 & -0.191 \\
\hline High Copper & -0.134 & 2 & 0.028 & -0.134 & High Copper & -0.064 & 2 & 0.022 & -0.064 \\
\hline \multicolumn{10}{|c|}{ Chione } \\
\hline \multicolumn{5}{|c|}{ Algae Depletion } & \multicolumn{5}{|c|}{ Algae Depletion } \\
\hline Tank & Mean & $\underline{N}$ & Standard Dev. & Median & Tank & Mean & $\underline{N}$ & Standard Dev. & Median \\
\hline Low Copper & 0.137 & 2 & 0.282 & 0.137 & Low Copper & 1.156 & 2 & \begin{tabular}{|l|}
0.104 \\
\end{tabular} & 1.156 \\
\hline Medium Copper & 0.202 & 2 & 0.499 & 0.202 & Medium Copper & 1.345 & 2 & 0.139 & 1.345 \\
\hline High Copper & 0.426 & 2 & 0.962 & 0.426 & High Copper & 0.913 & 2 & 0.398 & 0.913 \\
\hline \multicolumn{10}{|c|}{ Venerupis } \\
\hline \multicolumn{5}{|c|}{ Algae Depletion } & \multicolumn{5}{|c|}{ Algae Depletion } \\
\hline Tank & Mean & $\underline{\underline{N}}$ & Standard Dev. & Median & Tank & Mean & $\underline{\mathrm{N}}$ & Standard Dev. & Median \\
\hline Low Copper & 1.360 & 2 & 0.332 & 1.360 & Low Copper & 0.799 & 2 & 0.084 & 0.799 \\
\hline Medium Copper & 0.363 & 2 & 0.846 & 0.363 & Medium Copper & 1.605 & 2 & 0.169 & 1.605 \\
\hline High Copper & 1.383 & 2 & 0.532 & 1.383 & High Copper & 1.389 & 2 & 0.458 & 1.389 \\
\hline \multicolumn{10}{|c|}{ Musculista } \\
\hline \multicolumn{5}{|c|}{ Algae Depletion } & \multicolumn{5}{|c|}{ Algae Depletion } \\
\hline Tank & Mean & $\underline{\mathrm{N}}$ & Standard Dev. & Median & Tank & Mean & $\underline{\mathrm{N}}$ & Standard Dev. & Median \\
\hline Low Copper & -0.080 & 2 & 0.049 & -0.080 & Low Copper & -0.407 & 2 & 0.024 & -0.407 \\
\hline Medium Copper & -0.048 & 2 & 0.013 & -0.048 & Medium Copper & -0.226 & 2 & 0.159 & -0.226 \\
\hline High Copper & -0.206 & 2 & 0.065 & -0.206 & High Copper & -0.297 & 2 & 0.053 & -0.297 \\
\hline
\end{tabular}

A

\begin{tabular}{|c|c|c|c|c|c|c|c|c|c|}
\hline \multicolumn{10}{|c|}{12 Day } \\
\hline \multicolumn{5}{|c|}{ Day 0} & \multicolumn{5}{|c|}{ Day 12} \\
\hline \multicolumn{10}{|c|}{ Control } \\
\hline \multicolumn{5}{|c|}{ Algae Depletion } & \multicolumn{5}{|c|}{ Algae Depletion } \\
\hline Tank & Mean & $\underline{\mathrm{N}}$ & Standard Dev. & Median & Tank & Mean & $\underline{\mathrm{N}}$ & Standard Dev. & Median \\
\hline Low Copper & -0.255 & 2 & 0.076 & -0.255 & Low Copper & 0.113 & 2 & 0.025 & 0.113 \\
\hline Medium Copper & 0.249 & 2 & 0.193 & 0.249 & Medium Copper & 0.155 & 2 & 0.102 & 0.155 \\
\hline High Copper & 0.384 & 2 & 0.276 & 0.384 & High Copper & 0.321 & 2 & 0.004 & 0.321 \\
\hline \multicolumn{10}{|c|}{ Chione } \\
\hline \multicolumn{5}{|c|}{ Algae Depletion } & \multicolumn{5}{|c|}{ Algae Depletion } \\
\hline Tank & Mean & $\underline{\mathrm{N}}$ & Standard Dev. & Median & Tank & Mean & $\underline{\mathrm{N}}$ & Standard Dev. & Median \\
\hline Low Copper & $\overline{1.128}$ & $\overline{2}$ & 0.016 & $\overline{1.128}$ & Low Copper & $\overline{0.546}$ & $\overline{2}$ & 0.235 & $\overline{0.546}$ \\
\hline Medium Copper & 0.688 & 2 & 0.201 & 0.688 & Medium Copper & 0.596 & 2 & 0.117 & 0.596 \\
\hline High Copper & 0.731 & 2 & 0.105 & 0.731 & High Copper & 0.686 & 2 & 0.354 & 0.686 \\
\hline \multicolumn{10}{|c|}{ Venerupis } \\
\hline \multicolumn{5}{|c|}{ Algae Depletion } & \multicolumn{5}{|c|}{ Algae Depletion } \\
\hline Tank & Mean & $\underline{N}$ & Standard Dev. & Median & Tank & Mean & $\underline{\mathrm{N}}$ & Standard Dev. & Median \\
\hline Low Copper & 1.320 & 2 & 0.055 & 1.320 & Low Copper & 1.154 & 2 & 0.217 & 1.154 \\
\hline Medium Copper & 0.552 & 2 & 0.546 & 0.552 & Medium Copper & 0.788 & 2 & 0.106 & 0.788 \\
\hline High Copper & 0.984 & 2 & 0.208 & 0.984 & High Copper & 0.869 & 2 & 0.146 & 0.869 \\
\hline \multicolumn{10}{|c|}{ Musculista } \\
\hline \multicolumn{5}{|c|}{ Algae Depletion } & \multicolumn{5}{|c|}{ Algae Depletion } \\
\hline Tank & Mean & $\underline{N}$ & Standard Dev. & Median & Tank & Mean & $\underline{\mathrm{N}}$ & Standard Dev. & Median \\
\hline Low Copper & 0.482 & 2 & 0.204 & 0.482 & Low Copper & 0.523 & 2 & 0.127 & 0.523 \\
\hline Medium Copper & 0.320 & 2 & 0.167 & 0.320 & Medium Copper & 0.470 & 2 & 0.299 & 0.470 \\
\hline High Copper & 0.579 & 2 & 0.182 & 0.579 & High Copper & N/A & 0 & $\mathrm{~N} / \mathrm{A}$ & $\mathrm{N} / \mathrm{A}$ \\
\hline
\end{tabular}


Table 7.2 Survivorship comparison between this study and published research. Juhl $2018 \mathrm{ppb}$ values represent calculated copper concentration values, with $0 \mathrm{ppb}$ representing "Low Copper", 25 ppb as "Medium Copper" and 50 ppb as "High Copper".

\begin{tabular}{|c|c|c|c|c|}
\hline \multicolumn{5}{|c|}{ Juhl 2018} \\
\hline Species & Native/NIS & $\mathrm{ppb}$ & Survivorship Ratio & Duration \\
\hline C. californiensis & Native & 0 & 0.933 & 18 days \\
\hline C. californiensis & Native & 0 & 0.933 & 12 days \\
\hline V. philippinarum & NIS & 0 & 1.000 & 18 days \\
\hline V. philippinarum & NIS & 0 & 1.000 & 12 days \\
\hline M. senhousia & NIS & 0 & 0.933 & 18 days \\
\hline M. senhousia & NIS & 0 & 1.000 & 12 days \\
\hline C. californiensis & Native & 25 & 0.867 & 18 days \\
\hline C. californiensis & Native & 25 & 1.000 & 12 days \\
\hline V. philippinarum & NIS & 25 & 0.867 & 18 days \\
\hline V. philippinarum & NIS & 25 & 0.800 & 12 days \\
\hline M. senhousia & NIS & 25 & 0.933 & 18 days \\
\hline M. senhousia & NIS & 25 & 0.600 & 12 days \\
\hline C. californiensis & Native & 50 & 0.733 & 18 days \\
\hline C. californiensis & Native & 50 & 0.467 & 12 days \\
\hline V. philippinarum & NIS & 50 & 0.267 & 18 days \\
\hline V. philippinarum & NIS & 50 & 0.200 & 12 days \\
\hline M. senhousia & NIS & 50 & 0.267 & 18 days \\
\hline M. senhousia & NIS & 50 & 0.067 & 12 days \\
\hline \multicolumn{5}{|c|}{ Grout and Levings 2001} \\
\hline Species & Native/NIS & $\mathrm{ppb}$ & Survivorship Ratio & Duration \\
\hline M. edulis & NIS & $<5.0$ & 0.000 & 41 days \\
\hline M. edulis & NIS & 5.5 & 0.18 & 41 days \\
\hline M. edulis & NIS & 6.8 & 0.85 & 41 days \\
\hline M. edulis & NIS & 7.4 & 0.97 & 41 days \\
\hline M. edulis & NIS & 8.8 & 0.25 & 41 days \\
\hline M. edulis & NIS & 8.8 & 0.25 & 41 days \\
\hline M. edulis & NIS & 9.8 & 0.88 & 41 days \\
\hline M. edulis & NIS & 12.3 & 0.36 & 41 days \\
\hline M. edulis & NIS & 13 & 0.04 & 41 days \\
\hline M. edulis & NIS & 67.5 & 0.21 & 41 days \\
\hline M. edulis & NIS & 95.6 & 0.42 & 41 days \\
\hline M. edulis & NIS & 765.2 & 0.11 & 41 days \\
\hline
\end{tabular}


Table 7.3 Growth comparison between this study and previously published research. Juhl 2018 ppb values represent calculated copper concentration values, with 0 ppb representing "Low Copper", 25 ppb corresponding to "Medium Copper" and 50 ppb corresponding to "High Copper".

\begin{tabular}{|c|c|c|c|c|c|}
\hline \multicolumn{6}{|c|}{ Juhl 2018} \\
\hline Species & Native/NIS & $\mathrm{ppb}$ & Growth \% & Growth/day (mm) & Duration \\
\hline C. californiensis & Native & 0 & 0.217 & 0.0058 & 12 days \\
\hline V. philippinarum & NIS & 0 & 0.106 & 0.0033 & 12 days \\
\hline M. senhousia & NIS & 0 & 0.506 & 0.0067 & 12 days \\
\hline C. californiensis & Native & 25 & 0.122 & 0.0033 & 12 days \\
\hline V. philippinarum & NIS & 25 & 0.137 & 0.0042 & 12 days \\
\hline M. senhousia & NIS & 25 & 0.324 & 0.0042 & 12 days \\
\hline C. californiensis & Native & 50 & 0.176 & 0.0050 & 12 days \\
\hline V. philippinarum & NIS & 50 & 0.125 & 0.0042 & 12 days \\
\hline M. senhousia & NIS & 50 & 0.440 & 0.0058 & 12 days \\
\hline \multicolumn{6}{|c|}{ Peterson 1982} \\
\hline Species & Native/NIS & $\mathrm{ppb}$ & Growth \% & Total Growth (mm) & Duration \\
\hline C. undatella & Native & & & 0.03 & Summer 1975 \\
\hline C. undatella & Native & & & 1.05 & Winter 1975 \\
\hline C. undatella & Native & & & 0.07 & Summer 1976 \\
\hline C. undatella & Native & & & 0.46 & Winter 1976 \\
\hline \multicolumn{6}{|c|}{ Grout and Levings 2001} \\
\hline Species & Native/NIS & $\mathrm{ppb}$ & Growth \% & Growth/day (mm) & Duration \\
\hline M. edulis & NIS & $<5.0$ & & 0.0086 & 41 days \\
\hline M. edulis & NIS & 5.5 & & 0.0057 & 41 days \\
\hline M. edulis & NIS & 6.8 & & 0.0086 & 41 days \\
\hline M. edulis & NIS & 7.4 & & 0.0514 & 41 days \\
\hline M. edulis & NIS & 8.8 & & 0.0000 & 41 days \\
\hline M. edulis & NIS & 8.8 & & 0.0029 & 41 days \\
\hline M. edulis & NIS & 9.8 & & 0.0171 & 41 days \\
\hline M. edulis & NIS & 12.3 & & -0.0014 & 41 days \\
\hline M. edulis & NIS & 13 & & 0.0014 & 41 days \\
\hline M. edulis & NIS & 67.5 & & -0.0014 & 41 days \\
\hline M. edulis & NIS & 95.6 & & 0.0000 & 41 days \\
\hline M. edulis & $\mathrm{NIS}$ & 765.2 & & -0.0043 & 41 days \\
\hline
\end{tabular}


Table 7.4 Mucus production comparison between this study and published research. Juhl 2018 ppb values represent calculated copper concentration values, with 0 ppb representing "Low Copper", 25 ppb corresponding to "Medium Copper" and 50 ppb corresponding to "High Copper".

\begin{tabular}{|c|c|c|c|c|}
\hline \multicolumn{5}{|c|}{ Juhl 2018} \\
\hline Species & Native/NIS & $\mathrm{ppb}$ & Mucus/Dry Mass $(\mathrm{g} / \mathrm{g})$ & Duration \\
\hline C. californiensis & Native & 0 & 0.184 & 18 days \\
\hline C. californiensis & Native & 0 & 0.485 & 12 days \\
\hline V. philippinarum & NIS & 0 & 0.11 & 18 days \\
\hline V. philippinarum & NIS & 0 & 0.223 & 12 days \\
\hline M. senhousia & NIS & 0 & 0.242 & 18 days \\
\hline M. senhousia & NIS & 0 & 0.143 & 12 days \\
\hline C. californiensis & Native & 25 & 0.085 & 18 days \\
\hline C. californiensis & Native & 25 & 0.138 & 12 days \\
\hline V. philippinarum & NIS & 25 & 0.09 & 18 days \\
\hline V. philippinarum & NIS & 25 & 0.348 & 12 days \\
\hline M. senhousia & NIS & 25 & 0.184 & 18 days \\
\hline M. senhousia & NIS & 25 & 0.109 & 12 days \\
\hline C. californiensis & Native & 50 & 0.385 & 18 days \\
\hline C. californiensis & Native & 50 & 0.058 & 12 days \\
\hline V. philippinarum & NIS & 50 & 0.111 & 18 days \\
\hline V. philippinarum & NIS & 50 & 0.016 & 12 days \\
\hline M. senhousia & NIS & 50 & 0.488 & 18 days \\
\hline M. senhousia & NIS & 50 & 0 & 12 days \\
\hline \multicolumn{5}{|c|}{ Sze and Lee 1995} \\
\hline Species & Native/NIS & $\mathrm{ppb}$ & Mucus/Dry mass $(\mathrm{g} / \mathrm{g})$ & Duration \\
\hline P. viridis & & 0 & 0.004 & 3 months \\
\hline S. virgatus & & 0 & 0.002 & 3 months \\
\hline P. viridis & & 50 & 0.011 & 3 months \\
\hline S. virgatus & & 50 & 0.004 & 3 months \\
\hline \multicolumn{5}{|c|}{ Vosloo et al 2012} \\
\hline Species & Native/NIS & $\mathrm{ppb}$ & Mucus/Dry Mass (g/kg/min) & Duration \\
\hline P. perna & NIS & 0 & 0.015 & 1 day \\
\hline P. perna & NIS & 12.5 & 0.02 & 1 day \\
\hline P. perna & NIS & 25 & 0.03 & 1 day \\
\hline P. perna & NIS & 37.5 & No Data & 1 day \\
\hline P. perna & NIS & 50 & 0.045 & 1 day \\
\hline
\end{tabular}




\section{APPENDIX D: Pilot Study Data}

Table 8.1: Survivorship data from the pilot study examining survivorship percentage in relation to copper exposure.

\begin{tabular}{|c|c|c|c|c|}
\hline & \multicolumn{4}{|c|}{ Chione } \\
\hline Estimated Cu concentration & $0 \mathrm{ppb}$ & $25 \mathrm{ppb}$ & $50 \mathrm{ppb}$ & $100 \mathrm{ppb}$ \\
\hline Initial $n$ & 15 & 15 & 15 & 15 \\
\hline Final $n$ & 15 & 15 & 8 & 1 \\
\hline Survivorship \% & $100.00 \%$ & $100.00 \%$ & $53.33 \%$ & $6.67 \%$ \\
\hline & \multicolumn{5}{|c|}{ Musculista } \\
\hline Estimated Cu concentration & $0 \mathrm{ppb}$ & $25 \mathrm{ppb}$ & $50 \mathrm{ppb}$ & $100 \mathrm{ppb}$ \\
\hline Initial $n$ & 15 & 15 & 15 & 15 \\
\hline Final $n$ & 15 & 11 & 1 & 0 \\
\hline Survivorship \% & $100.00 \%$ & $73.33 \%$ & $6.67 \%$ & $0.00 \%$ \\
\hline & \multicolumn{5}{|c|}{ Venerupis } \\
\hline Estimated Cu concentration & $0 \mathrm{ppb}$ & $25 \mathrm{ppb}$ & $50 \mathrm{ppb}$ & $100 \mathrm{ppb}$ \\
\hline Initial $n$ & 15 & 15 & 15 & 15 \\
\hline Final $n$ & 15 & 11 & 4 & 0 \\
\hline Survivorship \% & $100.00 \%$ & $73.33 \%$ & $26.67 \%$ & $0.00 \%$ \\
\hline
\end{tabular}

